Irrigation and

Streamflow Depletion in Columbia River Basin

above The Dalles, Oregon By W. D. SIMONS

GEOLOGICAL SURVEY WATER-SUPPLY PAPER 1220

An evaluation of the consumptive use of water based on the amount of irrigation

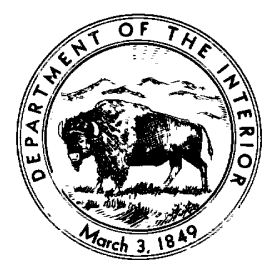




\section{UNITED STATES DEPARTMENT OF THE INTERIOR}

\section{Douglas McKay, Secretary}

\section{GEOLOGICAL SURVEY}

W. E. Wrather, Director 


\section{CONTENTS}

Abstract.

Introduction

Purpose and scope..

Acknowledgments.

Irrigation in the basin.

Historical summary.

Legislation.

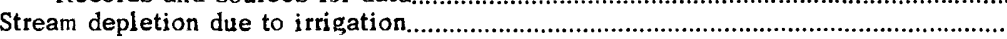

Consumptive use by crops.

Methods of measurement.

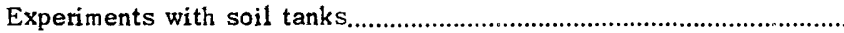

Lysimeter experiments.

Experiments with field plots.

Determinations of soil moisture.

Fluctuations of the water table.

Studies of inflow and outflow..

Estimates of average consumptive use.

Irrigated areas and estimated streamflow depletion.

Columbia River above the international boundary.

Kootenai River basin.

Pend Oreille River basin.

Clark Fork above Missoula, Mont

Clark Fork below Missoula, Mont

Clark Fork at St. Regis, Mont.

Flathead River.

Pend Oreille River below $Z$ Canyon near Metaline Falls, Wash..........

Columbia River at international boundary

Columbia River, international boundary to Grand Coulee Dam.

Kettle River.

Colville River.

Spokane River

Columbia River at Grand Coulee Dam, Wash

Columbia River, Grand Coulee Dam to Snake River.

Okanogan River.

Methow River.

Chelan River.

Entiat River.

Wenatchee River.

Columbia River at Trinidad.

Crab Creek.

Yakima River.

Columbia River, between Yakima River and Snake River............................ 57

Snake River basin.

Snake River above Heise.

Snake River, Heise to King Hill

Henrys Fork.

Portneuf River...........

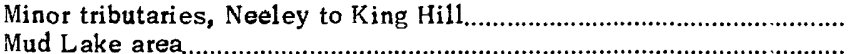

Little Lost River.

Big Lost River...

Big Wood River. 
Irrigated areas and estimated streamflow depletion-Continued

Snake River basin-Continued

Snake River, Heise to King Hill-Continued

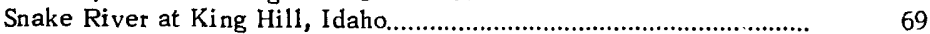

Snake River, King Hill to mouth......................................................... 70

Bruneau River......

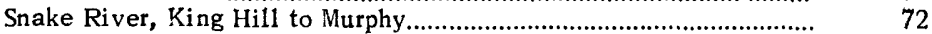

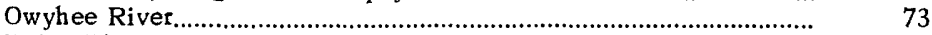

Boise River...................................................................................... 78

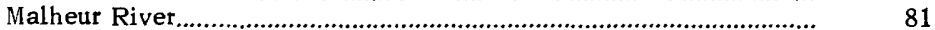

Payette River............................................................................... $\quad 84$

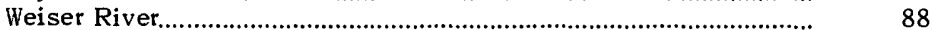

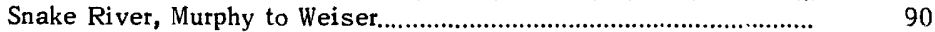

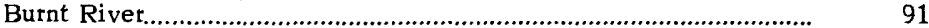

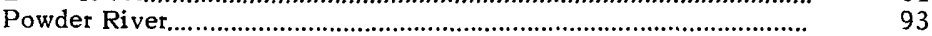

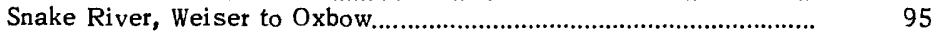

Pine Creek...................................................................................... 96

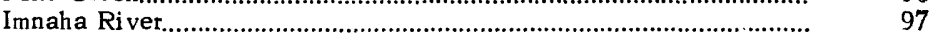

Salmon River.................................................................................. 99

Grande Ronde River................................................................... $\quad 102$

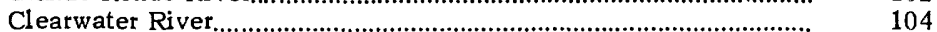

Snake River, Oxbow to Clarkston.................................................... 106

Columbia River, Snake River to The Dalles................................................... 108

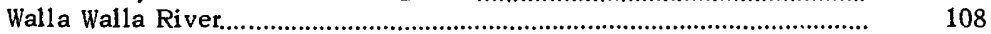

Umatilla River.................................................................................... 110

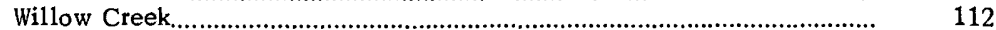

John Day River................................................................................... 113

Deschutes River................................................................................. 116

Columbia River neat The Dalles, Oreg................................................... 121

References cited ................................................................................................ 123

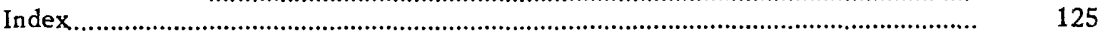

\section{ILLUSTRATIONS}

Plate 1. Map showing drainage basin of Columbia River above The Dalles,

Oreg............................................................................................ In pocket

Figure 1. Graph showing irrigated area, above selected points in the

Columbia River basin.

\section{TABLES}

Table 1. Net consumptive use, by subdivisions, of the Columbia River $k$ asin.... 


\title{
IRRIGATION AND STREAMFLOW DEPLETION IN COLUMBIA RIVER BASIN ABOVE THE DALLES, OREGON
}

\author{
By W. D. Simons
}

\begin{abstract}
The Columbia River is the largest stream in western United States. Above The Dalles, Oregon, it drains an area of 237,000 square miles, of which 39,000 squar? miles is in Canada. This area is largely mountainous and lies between the Rocky Mountains and and the Cascade Range. The Kootenai, Pend Oreille, and Snake Rivers are the principal tributaries. Precipitation varies from 7 inches near Kennewick, Wash. to ov in some of the mountainous regions. Most of the runoff occurs in the spring and summer months as a result of melting snow. Precipitation is generally light durin? the summer months, and irrigation is necessary for sustained crop production.
\end{abstract}

Historical data indicate that irrigation in the Columbia River basin b:gan prior to 1840 at the site of missions established near Walla Walla, Wash. and Lewiston, Idaho. During the next half century the increase in irrigated area was slow and by 1890 included only 506,000 acres. The period 1890 to 1910 was marked by phenom inal increase to a total of $2,276,000$ acres in 1910 . Since that time there has been more gradual addition to a total of 4,004,500 acres of irrigated land in 1946 in the Columbia River basin above The Dalles, Oreg. Of this total 918,000 acres were located in the Cclumbia Basin above the mouth of the Snake River; 2,830,000 acres in the Snake River basin, and the balance, 256,000 acres below the mouth of the Snake River.

Values of net consumptive use were determined or estimated for various tributary basins of the Columbia River basin and compared to available experimenta' data. These values were then used to compute the average depletion which could be directly attributed to irrigation. The yield of a drainage basin was considered to be the sum of the observed runoff and the estimated depletion. For purposes of comparison, the depletion was expressed both in terms of acre-feet and as a percentage of the yield of the basin. This percentage depletion varied from less than 1 percent for many tributary basins to 53 percent for the portion of the Snake River basin between Heise and Kin? Hill, Idaho. For the Columbia River near The Dalles, Oreg., the average depletion dur: ng the period 1921 through 1945, amounted to 4.7 percent of the yield and the depletion ropresented by the 1946 stage of irrigation development amounted to 5.3 percent of the long-term yield. 


\section{INTRODUCTION}

\section{PURPOSE AND SCOPE}

Safe and economical design of a water-use project requires adequate knowledge of the available water supply. The hydrologic data necessary to determine the probable future water supply must be collected over a period of many years in order to adequately evaluate the effects of the vagaries of the weather and to determine the dependable yield during drought period. If during the period of investigation changes have occurred in the use of water, the effects of these changes must be considered in analyzing the data to determine probable future supply. Records of runoff covering a period of many years should be adjusted to a comparable level of water-supply development and water use.

Irrigation is one use of water that has been continually changing. The object of this report is to trace the enlargement in irrigated area in the various subdivisions and to evaluate the net depletion in streamflow resulting from increased irrigetion. Thus it presents some data necessary for the proper interpretation of runoff records used in water-supply studies.

Data on irrigated area presented herein was gathered from many sources. Information from any one source was never complete and was in many instances in conflict with informaticn from another source. Thus it became necessary to resolve trese differences and to estimate the missing periods. However, it is believed that the inaccuracies from these sources are, or the whole, relatively minor and that the data on irrigated area are sufficiently accurate for use in this study.

The adequacy of the water supply for specific areas is seldom known for a particular year. Likewise in the determination of net consumptive use, records are inadequate for estimating rates for individual years but are believed to be reliable when taken as an average over a period of years. Consumptive use will be greater during dry, hot summers, and a greater part of the water must be supplied by irrigation during these years. In a general study such as this, available data did not warrant an attempt to determine the net depletion, except on the basis of average annual rates for each part of the basin.

In the absence of reliable data on the ground-water conditions underlying these irrigated areas, assumptions had to be made as to locations where return flow would be recaptured by gaged streams. No allowance was made for water temporarily or permanently detained in ground-water storage. 
Only the United States portion of the basin has been considered in this report. Irrigation in Canada is relatively unimportant, except in the Okanogan River basin. Reference to irrigation in Canada is made in the discussions of individual tributary basins.

It is noteworthy that for most tributary basins the net depletion is a minor fraction of the yield of that basin, but this is no warranty that much greater supplies can be developed easily and cheaply. Indeed, the current use of water may be equal to or greater than the supply during the low-water season, even though the depletion is so small a fraction of the annual yield.

\section{ACKNOWLEDGMENTS}

The records of irrigated area included in this report were collected from many sources through the generous cooperation and assistance of many Federal, State, and local agencies, watermasters, county agents, lawyers, and private citizens. Reports of the Bureau of the Census and the Bureau of Reclamation were used extensively. The collection of detailed data on irrigation was performed by the following Geological Survey engineers: C. D. Bue for Montana, Lynn Crandall for upper Snake River, R. W. Revell for the remainder of Idaho, A. P. Lyman for Oregon, and G. L. Bodhaine for Washington. This report was prepared under the direct supervision of C. C. McDonald, staff engineer, Technical Coordination Branch, Tacoma, Wash. and under the administrative direction of $R$. W. Davenport, chief, Technical Coordination Branch, Washington, D. C.

\section{IRRIGATION IN THE BASIN}

\section{HISTORICAL SUMMARY}

The development of irrigation in the Columbia River basin has been intimately associated with the settlement and industrial development of the Northwest. Most of the basin suitable for crop production is semiarid, and irrigation is required during the growing season. Irrigation agriculture was practiced by the early settlers to meet the local needs for vegetable and grain crops. Transportation was slow and difficult, requiring each settlement to be practically self-sustaining. As settlement of the Northwest progressed, markets for farm products expanded continually, and irrigation agriculture became an increasing factor in the region's economy. 
The early settlers found ample opportunity to divert water from tributary streams to adjacent rich bottom or bench lands with little work and expense. These lands were well adapted to irrigation; they were level or gently rolling, had good natural drainage and were covered with grasses and sagebrush, which made preparation of the land for cultivation possible at low cost.

Probably the earliest irrigation in the Columbia River basin was carried on by the missionaires who settled in the basins of Walla Walla River and Clearwater River. In the fall of $18 £ \subseteq$ missions were located near Walla Walla, Wash. by Dr. Whitman and near Lewiston, Idaho by Dr. Spalding. The following year crops were grown at these missions, using water from Doan and Lapwai Creeks.

In the upper Columbia River basin, the earliest known irrigation was at St. Mary's Mission, on the Bitterroot River, about thirty miles south of Missoula, Mont. In about 1846 or 1847 the mission is reported (Bancroft, H. H., 1890, p. 604) to have produced wheat, potatoes, and other vegetables by irrigation from two small creeks running throughits farm. In 1854 Father DeSmet founded St. Ignatius' Mission about 32 miles north of I Missoula in the Flathead River basin, and irrigation probably was started during the following year.

There is an unauthenticated report of irrigation in the Yakima River basin, Washington antedating the Treaty of 1855 (House Doc. 103, vol. 2, 1934, p. 879). However it is probable that the first development came in 1867 when the Nelson Ditch was constructed to divert water from the Naches River. (Parker and Storey, 1917, p. 28.) This was 2 years after Yakima County was created in the Territory of Washington and about 6 years after the first pioneers had settled in this valley.

In other parts of the Columbia River basinsettlers were beginning to irrigate in order to meet the greatly increased demand for food crops created by the influx of settlers accompanying the booming mining and cattle raising activities during the period 1860 to 1880. During this period the railroads also were pushing westward, bringing additional settlers and expanding the markets for farm products.

In 1870 there were only 129,877 people in Washington, Oregon, and Idaho, and approximately 48,000 acres of land wers being irrigated in the Columbia River basin above The Dalles, Oreg. The most rapid increase in population during the period 1870 to 1880 occurred west of the Cascade Range. By 1880 the population was 300,949 , the greatest increase having occurred in the Willamette Valley in Oregon. 
The decade from 1880 to 1890 was one of intensive development in the Northwest. Transcontinental rail connections between the Great Lakes region and the ocean ports of the Pacific Northwest were completed in 1883 , opening vast new markets for the products of the lands, mines, and forests of the Northwest. Large irrigation projects were undertaken in the Snake and Yakima River valleys, and the population of Washington, Oregon, and Idaho increased 170 percent.

The rate of population increase during the period 1890 to 1900 was much below that of previous decades, reflecting the influence of the panic of 1893, but the total numerical increase of 329,927 was about twice that of the preceding decade. The irrigated area in the Columbia River basin above The Dalles, Oreg., meanwhile was expanding rapidly. From a total of 48,000 acres in 1870 , it has increased to 153,000 acres in 1880 and 506,000 acres in 1890 . (See page 122.) During the period 1884 to 1896 construction was commenced on practically all the canals that presently serve the lands above American Falls on the upper Snake River.

During the next two decades, 1890 to 1910 , the exparsion of irrigated area was phenomenal, reaching a total of 2,276,000 acres in 1910. Irrigation agriculture was firmly established as an important part of the economy of the basin east of the Cascade Range. Export markets became available and the agricultural economy was no longer geared to local needs. Aided by the above-normal rainfall during this period, dry farming, chiefly the raising of wheat, developed rapidly. Favorable Federal and State legislation and the participation of the Federal Government in water resource development (see p. 6) provided impetus to irrigation development, making possible the creation of irrigation districts.

Subsequent to 1920, the increase in irrigated lands has been gradual though in some of the smaller basins the increase amounts to a substantial part of the total irrigated area in these basins. The gradual change from the wet period of 1890-1910 to the dry period, which reached its most critical stage during the period 1929 to 1934 in most parts of the basin, had a substantial effect on irrigation developments. Many irrigation districts found that their storage reserves were inadequate to cope with such conditions and supplemental water supplies or additional storage capacity was needed. Additional irrigation projects were more expensive to develop, water supplies were less accessible than those for earlier projects, and pumping was often required. These factors tended to restrict irrigation expansion, especially during the years of the business depression. Recent irrigation activities generally have been limited to extensions of earlier projects, to providing supplemental water supplies, or to a combination with power projects that provide low-cost power for pumping. The 
great Columbia Basin Project incentral Washington, now in process of construction, is an example of the latter type of development. Power for the pumping of stored irrigation water for more than a million acres of land will be provided by Grand Coulee Dam.

In 1946 the irrigated area in the Columbia River basin above The Dalles, Oreg. totaled 4,004,500 acres. Of this total 918,300 acres were located above the mouth of the Snake River, 2,829,700 acres within the Snake River basin, and the balance of 256,500 acres below the mouth of the Snake River. The rate of expansion of irrigation is shown graphically by figure 1. From the motest beginnings made by the missionaries over a hundred years ago, irrigation has become the dominating factor in the agricultural economy of the Columbia River basin.

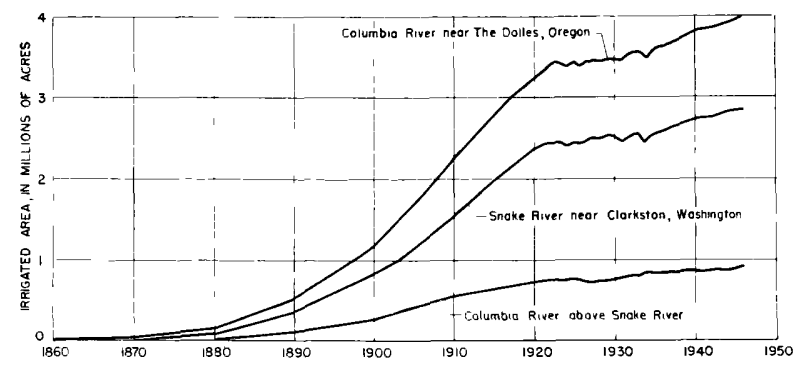

Figure 1. -Graph showing irrigated area, above selected points in the Columbia River basin.

\section{LEGISLATION}

The Federal and State laws relating to water rights and control of the public lands have had great influence upon the development of irrigation in the arid West. Some of this legislation was a matter of necessity in settling disputes arising from conflicting uses of water; some was designed specifically to stimulate irrigation.

The English common law recognizes the riparian doctrine. This doctrine vests in the owner of the land adjacent to a stream, the right to have the stream flow past his land in perpetuity, undiminished in quantity and unimpaired in quality. The riparian rule originated in England in a humid climate and was adapted to conditions and common uses in that country and in the eastern United States, but it proved to be inappropriate for conditions requiring the use of water for irrigation. The riparian doctrine gave rise to many disputes in the early days of development of the West. 
The doctrine of appropriation recognizes the principle that the first to put the water to beneficial use has a right to the water supericr to all later appropriations. This doctrine was essentially developed among the gold miners of California, and it became law through the sanction of the local courts. Most of the land in the arid and semiarid region was originally public land, and under the riparian doctrine the ownership of the streams was an accompaniment of the ownership of the land. In the Act of 1886 , the Federal Government recognized the water rights existing under local customs, laws, and decisions of courts and the right to construct ditches across public lands. The necessity of diverting water from the streams to irrigate lands distant from the streams was recognized and specifically provided for in the Desert Land Act of March 3, 1877, which required irrigation as a ccndition of land ownership. This law required a dedication of the water by its owner to the uses of irrigation, except where expressly forbidden in the case of navigable streams. These policies were later incorporated in the Reclamation Act in 1902 and are still in effect.

Until comparatively recent years, the title to irrigation water in some localities was in a chaotic state, but the acceptance of the doctrine of appropriation by substantially all the arid states and the early recognition by the Federal Government of State authority over nonnavigable waters have tended to eliminate most of the difficulties. In 1939 the Idaho Supreme Court stated: "The right of riparian ownership has been abrogated in Idaho." (National Resources Planning Board, 1943, p. 101.) In the years between 1921 and 1925, court decisions in Montana left no doult that the appropriation doctrine prevails in the State. Montana is the only State in the Columbia River basin that has not provided by statute for centralized control over the appropriation and administration of water. Although the Oregon Water Code of 1909 recognized riparian rights to some degree, the effect of court decisions and later statutes is to make the riparian doctrine little more than legal fiction in Oregon. (National Resources Planning Board, 1943, p. 107.) The Common Law doctrine of riparian rights is in force in Washington but "has been stripped of some of its rigors" particularly during the past 20 to 25 years, (National Resources Planning Board, 1943, p. 110) so that today it is of minor importance in the irrigation economy of the State.

The Carey Act of 1894 was designed to stimulate irrigation development in the arid states containing public lands by providing for the transfer of not more than 1,000,000 acres of arid public land to each State for development. Title to the land was acquired by individuals through State sponsorship with the requirement that the land be irrigated within 10 years after passage of the Act. This Act did much to stimulate development of irrigation esfecially in the Snake River basin in Idaho and Oregon. 
State authorization of irrigation districts in Washington, Oregon, and Idaho during the period 1890-95 contributed to the great increase of irrigation during this period.

The Reclamation Act of 1902 created the present Bureau of Reclamation and its Reclamation Fund and marked the keginning of Federal sponsorship of irrigation projects. By means of the Reclamation Fund the Federal Government may finance the construction of an irrigation project, and the costs are repaid by the land owners over a period of years. The Yakima Project, Washington and the Minidoka Project, Idaho were among the first undertaken by the Federal Government under the Reclamation Act. In 1948 the Tieton Irrigation District, Yakima Project, became the first Bureau project to liquidate its construction obligations (U. S. Bureau of Reclamation, 1948, p. 465).

\section{RECORDS AND SOURCES FOR DATA}

In general, "irrigated area" as used in this report is the gross farm area upon which water is artificially applied for the production of crops with no reduction for access roads, canals, or farm buildings. Native meadows and pasture lands are included if water is artificially applied during the year. This definitior is similar to the definition of irrigated land used by the Bureau of the Census: Land should be classed as irrigated which has water supplied to it for agricultural purposes by artificial means or by seepage from canals, reservoirs, or irrigated lands, but land which has natural ground water sufficiently near the surface to support plant life should not be classed as irrigated. Land which is flooded during high-water periods should be classed as irrigated if water is caused to flow over it by dems, canals, or other works, but should not be classed as irrigated if the overflow is due to natural causes alone.

Available data on irrigated areas are not entirely consistent with respect to the inclusion of land utilized for miscellaneous farm purposes other than the growing of irrigated crops. This report, however, uses the gross farm area on the premise that it would more accurately represent total consumptive use. This definition of irrigated area does not distinguish between lands having an adequate or inadequate supply of water.

Records are not sufficiently accurate or complete to reflect yearly fluctuations in the irrigated area in all tributary basins, and in many it has been necessary to estimate the acreage for years when the irrigated area could not be determined directly.

Determinations of irrigated area were based upon d $\varepsilon$ ta from as many independent sources as possible. The following scurces have been used: Census Reports, recorded water rights and court adjudications, Bureau of Reclamation reports, water-surply papers 
of the Geological Survey, County tax rolls, watermaster reports, records of irrigation companies and districts, State Fngineers' reports, publications of pioneer activities, Soil Conservation Service reports, and field reconnaissance.

A census of irrigation is available for $1889,1899,1902,1909$, $1919,1929,1934$, and 1939. Tabulations of irrigated areas by counties were of limited usefulness. During early years many county boundaries were changed so that much of the data for early years are not comparable with that for recent years except on a state-wide basis. Further, county lines do not coincide with topographic drainage divides so that for the purpose of this report, data by counties were useful only in a general way. Tabulations of irrigated area by drainage basins were mac'e in 1902, 1919,1929 , and 1939. These proved very useful in checking data from other sources although the drainage basin subdivisions used by the Bureau of the Census were seldom coincident with those of the stream-gaging stations.

The records concerning water rights and court adjudications were fruitful sources of data. Although many conflicts arose, commonly these records afforded the only information available for small or isolated areas and for early years. Data obtained from this source were used extensively to distribute the Census tabulations by tributary basins, especially in Idaho and Oregon. Usually they indicate the maximum amount of irrigated lands and have been so used for some areas.

The yearly and special reports of the Bureau of Reclamation contain complete records for projects constructed by that bureau; they afford data on the area actually supplied with water, and on the net area incultivation. Investigational reports on areas where projects were considered gave information relative to the stage of development existing at the time.

Water-supply papers of the Geological Survey also supplied data that could be used to spot check the tabulations of irrigated areas. These were primarily the reports on water utilization for specific areas, for example; Water utilization in the Snake River basin, Water-Supply Paper 657, and Water power of the Cascades, Yakima River basin, Water-Supply Paper 369 .

The tax records of many counties contain data on irrigated areas, owing to their higher appraisal value for tax purposes. However, for some parts of the basin information from this source was not entirely reliable and was used only in the absence of more accurate data. Montana, in compliance with an act passed by the legislature about 1919 requiring the classification of land in the State, 
made field surveys of all irrigated lands for tax purposes. From these land classification surveys the County totals were broken down by tributary basins. Although these breakdowns afnly only to the year in which the surveys were made, they providec' a guide for distributing county totals for other years. Since about 1920 , the Montana State Board of Equalization has published tabulations of irrigated area by counties in its biennial reports.

Reports of local watermasters, where available, were used in connection with material from other sources. In the Boise River valley and Snake River Plains such reports were used extensively.

The records kept by irrigation companies were utilized where available. In Washington each irrigation district formef under State law is required to submit a report annually to the Department of Conservation and Development, listing among other things the area irrigated during that year. These records were compiled and used extensively.

Various reports of the State agencies for Montana, Idaho, Oregon, and Washington were scanned for information. However, in most of these reports the information relating to irrigated area was not in sufficient detail to be useful for the purpose of this report. Field reconnaissance of many areas was necessary in order to complete the tabulations and to reconcile conflicting data from several sources. Farmers, lawyers, judges, bankers, pioneers, and other qualified persons were interviewed to obtain supplementary data. Conflicts between data from various sources have been resolved so far as possible, and any residual discrepancies are discussed under the appropriate area.

\section{STREAM DEPLETION DUE TO IRRIGATION}

The use of water to irrigate land and produce crops unavoidably reduces the amount of water available for other purposes. Usually the total amount of water diverted is considerably greater than the net loss to the basin, which is the amount dissipated through transpiration by crops and through evaporation from the land surfaces that have been artificially wetted. Most of the remaining undissipated water returns ultimately to the streams, largely by underground percolation at a time and rate unlike natural runoff. This return flow usually contains a higher concentration of dissolved minerals than does native river water, owing in part to its prolonged contact with the earth material through which it percolates and partly because it tends to accumulate the dissolved matter that is dissipated by plant growth. Thus, the use of weter for irrigation diminishes streamflow by increasing evapotranspiration, changes the regimen of the stream by altering runcff conditions, and commonly increases the mineral content of the water. 
Under natural conditions a portion of the available precipitation becomes soil water and meets the demand of evaporation and transpiration. The amount so consumed depends upon the amount and seasonal distribution of the precipitation, the concurrent temperatures, characteristics of the soil, and the types and amount of vegetation. Under irrigation, both the available soil water and the opportunity for evaporation and transpiration are greatly increased. More water is dissipated by transpiration of the irrigated vegetation, by evaporation from the artificially wetted soil, and by evaporation from artificial water surfaces such as reservoirs, canals, and waste-water swamps. The moisture available under natural conditions influences the extent and nature of the native vegetal cover. In the Columbia Basin, most of the land currently being irrigated was originally arid or semiarid, and little moisture was available to support native vegetation. Consequently the consumptive use by the native vegetation was relatively small. These same factors tend to make the net consumptive use by irrigated crops relatively large.

When irrigation is first begun in a new area, all potential return flow does not get back into the streams. Commonly part of this potential flow goes into building up ground-water storage in the area. An unstable condition is established that may require years to stabilize. Thus the amount and the seasonal distribution of the return flow depends not only upon the distance and the rate of percolation back to the stream but also upon the volume of water retained in ground-water storage. Streamflow characteristics may be further modified by storage in surface reservoirs and by evaporation from reservoirs and canals.

Large blocks of irrigated land, such as on the Snake River Plains, may exert some influence upon the climate of the region. However, this effect is believed to be relatively small and highly localized and has not been considered further in this report.

\section{CONSUMPTIVE USE BY CROPS}

The term "consumptive use" has been generally cccepted as synonymous with evapotranspiration, and represents the quantity of water absorbed and transpired in building plant tissue, together with that evaporated from the adjacent land surface. Investigators have found difficulty in separately measuring the water absorbed by plants and that evaporated from the land. Accordingly, these two components have been combined as "evapotranspiration." As the term applies to irrigated lands, consumptive use may be defined as the quantity of water in acre-feet per acre per year absorbed by the crop and transpired or used directly in building plant tissue, together with that evaporated directly from the crop- 
producing land. Definitions applicable to large irrigated areas or basins including bare ground and natural vegetation, includes water evaporated from snow and that intercepted and evaporated from vegetation. This report is concerned primarily with the consumptive use by crops, of the water applied to the land through irrigation. This quantity which has been called net consumptive use, is determined by deducting the estimated contribution ky rainfall toward the production of irrigated crops from the annual consumptive use.

The net consumptive use so determined is considered to represent the depletion of streamflow as a result of irrigation, disregarding evaporation from reservoirs and canals. Total depletion in a basin may be defined as the difference between precipitation and runoff, with appropriate allowance for changes in storage. No attempt is made in this report to estimate natural or total depletion, but only the depletion directly attributable to irrigation.

As used in this report, return flow is that portion of the irrigation water which returns at any time or place to a surface stream.

\section{METHODS OF MEASUREMENT}

Many methods of determining consumptive use have been used in hydrologic investigations: direct measurement on soil tanks, lysimeters, or field plots, determination and interprotation of fluctuations in soil moisture, evaluation of water table fluctuations, and inflow-outflow inventories on farms, irrigation projects, or valleys.

EXPERIMENTS WITH SOIL TANKS

Experiments with soil tanks have been used by many investigators because the construction of the se tanks is simple and the investment required is small. An unsealed tank is filled with undisturbed soil native to the area and set flush with the surrounding land surface. Evapotranspiration is determined by applying water only in such quantities as to satisfy the evapotranspirational demands. Thus the amount of water applied, including precipitation, is the consumptive use. The success of this type of experiment is limited by the ability of the experimenter to reproduce natural conditions. The tanks may be of any size or defth necessary to permit normal root development. The plant density should be representative of the area and the foliage of the plants should not extend beyond the walls of the tank. 
In 1930 the Duty of Water Committee of the American Society of Civil Engineers (Harding, 1930, pp. 1349-1377) called attention to the difficulties of reproducing natural conditions with tank experiments and indicated that the results may be of quistionable value. However, many recent experiments have been made using this method successfully.

In the upper Rio Grande Valley this method was used by Bureau of Agricultural Engineering, Department of Agriculture (Blaney and others, 1938, pp. 89-92) in connection with the integration method of determining streamflow depletion. In Idaho, Lynn Crandall used this method successfully in measuring net consumptive use ${ }^{1}$.

\section{LYSIMETER EXPERIMENTS}

The lysimeter method is similar to the soil-tank method. A lysimeter is essentially a tank in which natural soil conditions are retained or reproduced as nearly as possible, except that the bottom is enclosed and provision is made for measuring the water that may percolate below the root zone. Consumptive use is determined by weighing the entire lysimeter and contents on a suitable scale. The soil moisture may be brought up to field capacity at the beginning and the end of the experiment; the observed losses represent the consumptive use.

Soil tanks or lysimeters, equipped with Mariotte supply tanks to maintain a water table at a specified depth, have also been quite successful in measuring the water consumed by plants. The tank or lysimeter method has probably been more extensively used than any other method for determining the transpiration of individual plants or groups of plants, and for determining rates of infiltration, percolation, and related phenomena.

In the Columbia River basin, lysimeters were used by Bark (1916) to determine consumptive use for conditions in Idaho.

EXPERIMENTS WITH FIELD PLOTS

Field plots have been used extensively to determine consumptive use by crops growing under normal field conditions in undisturbed soil. The method is essentially identical to that used for soil tanks, in which moisture is applied in controlled amounts sufficient only to maintain soil moisture adequate for crop production. For successful operation, this method requiros a deep water table to prevent the transfer of water from the water table to the root zone by capillary action.

1 Crandall, Lynn, Report of use of water on Twin Falls North Side Project, unpublished, 1918.

279538 ○ $-53-2$ 
Field-plot experiments have been performed by numerous investigators, among them being Fortier and Young (1930) who made studies of monthly and seasonal net consumptive use in the Columbia River basin. Powers $(1914 ; 1917)$ used this method in studies of soil moisture and requirements for irrigation in Oregon. Field plots were also used by Hemphill (1922) in Colorado, Widstoe (1912) in Utah, and Snelson (1922) in Alberta, Canada.

\section{DETERMINATIONS OF SOIL MOISTURE}

The soil-moisture method has been used in connection with field-plot experiments and relies upon sampling the soil to trace the movement and dissipation of water applied to the soil. Samples are taken with a soil tube at various depths from the 1 nd surface to well below the root zone of the vegetal cover. This rethod provides a means of evaluating the amount of deep seepago, which is the variable element in the field plot method, as well as the determination of the optimum soil moisture for a given cover crop. As in the field plot experiments, this method also requires a deep water table to prevent transfer of water from the water table to the root zone by capillary action. Blaney (1930) is crsodited with developing this method in California, and it has beer used primarily by Blaney and his associates.

\section{FLUCTUATIONS OF THE WATER TABLE}

Where the water table is near the surface, so that the capillary fringe is within reach of the plant roots, the recorded fuctuations of the water table may be evaluated in terms of evapotranspiration losses. For successful application of this procedure, relatively flat areas are required and ground water flow must be at a minimum. Adjustments of the results for precipitation and irrigation are possible if the quantities of water applied are carefully measured. In areas of moderate to slight movement of ground water, daily consumptive use may be determined from the records of diurnal fluctuations of the water table.

The specific yield of the aquifer must be determined, and continuous records of the fluctuations of the water table must be obtained by installing test wells equipped with water-level recorders at representative locations in the area. The daily ground water replenishment to the area is based on the rise in ground water from midnight to $4 \mathrm{a} . \mathrm{m}$. when evapotranspiration losses are assumed to be zero. The daily consumptive use is determined by adjusting the daily ground water replenishment by the amount of water represented by the rise or fall in the water table during the 24-hour period. This method was developed by $W$. N. White (1932, pp. 87, 88) in his studies of the ground water in Escalante Valley, Utah. 
Consumptive use may be determined for irrigated farris or projects from inflow and outflow data. The inflow to the area is measured as precipitation, streamflow, canal diversions, and groundwater seepage. The outflow includes waste from the irrigation system, streamflow below the project, drainage or other return flow, and outflow of ground water. Changes in ground-water storage, soil moisture, and losses by deep seepage must be determined or must be kept to a minimum. These factors are sometimes difficult to determine and may invalidate the experiment unless adequately evaluated.

Many investigations have been made by the inflow-outflow method, several of them in the Columbia River basin. Lynn Crandall ${ }^{2}$ used this method in analyzing the water requirements of the North Side Twin Falls area. Similar studies were made by Lewis ${ }^{3}$ in the same general area. During 1916 and 1917 Steward and Paul used the inflow-outflow method in the course of drainage investigations in the lower valley of the Boise River. Recently the Bureau of Reclamation made a similar study in the lower Yakima River basin.

Under certain conditions an entire valley may be included in evapotranspiration studies. The primary requirement is that such a valley be underlain with impervious bedrock so that a minimum amount of water escapes through deep percolation. All the inflow, outflow, and storage factors must be measured, inclucing allowances for evaporation from water surfaces and wet lands and consumptive use by natural vegetation.

This type of study has been made by many investigators, including Lee (1912) in the Owens Valley, Ullrich in the Sevier River basin, Hemphill (1922) in the Cache La Poudre Valley, ard Parshall (1930, p. 961) in the South Platte River basin.

\section{ESTIMATES OF AVERAGE CONSUMPTIVE USE}

For maximum usefulness evapotranspiration data should be correlated with the basic causative factors, so that the data obtained at one location are applicable at another locality on a sound basis.

2 Crandall, Lynn, 1918, Report of use of water on Twin Falls North Side Projec:, unpublished.

${ }^{3}$ Lewis, M. R. , 1919, Experiments on the proper time and amount of study' of irrigation, Twin Falls Expert. Sta., 1914, 1915, and 1916, U. S. Dept. of Agriculture cooperating with Twin Falls County Commissioners, Twin Falls Canal Co., and Twin Falls Commercial Club.

4 Steward, W. G., and Paul, D. J. . 1919, Report on drainage investigations of Pioneer and Nampa-Meridian Districts in Boise Valley for the years 1916 and 1917, U. S. Bur. of Reclamation, unpublished.

5 Ullrich, C. J., Testimony at hearing of the Colorado River Comm. Salt Lake City, Utah, March 27, 28, 1922 . 
Of the studies to correlate evapotranspiration with known meteorologic conditions, the works of Lowry and Johnson (1942, p. 1243) and Blaney and Criddle ${ }^{6}$ are possibly the two host known examples. Both provide a means of estimating the probable consumptive use by irrigated crops.

Lowry and Johnson analyzed data on consumptive use from irrigated valleys and humid watersheds, representing a wide range in climate, latitude, elevation, and type of crops. Thay found a straightline relation between annual consumptive use and heat supply, expressed in terms of accumulated daily maximum temperatures above $32 \mathrm{~F}$. during the growing season. So far as possible data excluded deep percolation losses. As defined for the effectiveheat method, consumptive use is the quantity of water per year absorbed by the crop and transpired or used directly in building plant tissue, over the "equivalent valley area". The equivalent valley area in each case comprises the entire area or valley (except for areas using no streamflow), reduced to an area consuming water at a rate equivalent to that by the cropped area. The unit values of consumptive use derived by the effective-heat method represent estimated average annual consumptive use per acre of cropped land.

An adequate water supply is assumed in using this method and a mixture of the crops commonly grown on irrigation projects. The relationship was based upon studies made of twenty irrigated and natural valleys or areas where records of inflow and outflow were readily available or could be closely estimated. Records for individual areas ranged from 1 to 18 years in length. The records of years of inadequate water supply were adjusted to make them comparable with those for years having a normal water supply. The mean values of consumptive use and effective heat for each individual area were used to determine the relationship. The values for individual years were found to scatter through wide limits, but the average values for consumptive use anc heat supply plot on a fairly well defined curve. The use of the average values in the correlation removes the apparent inconsistencies of some individual years and tends to exaggerate the degree of correlation obtained. The results derived from the use of this relationship are general and are applicable only under average conditions of water supply, crop distribution, and irrigation practices.

To determine the net consumptive use that is supplied by irrigation water the "effective precipitation" must be deducted from the annual consumptive use. Effective precipitation is that portion of the precipitation which is estimated to be used by the grow-

\footnotetext{
${ }^{6}$ Blaney, Harry F., and Criddle, W. D. , A method of estimating water requi rements in irrigated areas from climatological data: Div, of Irrigation, Soil Conserv. Service, U. S. Dept. of Agr., revised Dec. 1947. (mimeographed)
} 
ing crops and depends upon the amount and intensity of the precipitation, type of soil, and rate at which soil water can be used by the plants.

Blaney and Criddle took a different approach to the determination of consumptive use. Their method is a modificaticn of methods used in the investigations in the upper Rio Grande (Blaney and others, 1938, pp. 293-423) and Pecos River (Blaney and others, 1942 , pp. 172-230). The authors demonstrated that consumptive use for each type of crop varies with the mean temperature and the number of daylight hours. A consumptive use factor is obtained by multiplying the mean temperature for the month by the percent of the total annual daylight hours that occur during the month. Consumptive use is assumed to vary directly as this factor. Using the notations shown below, this relationship can be expressed mathematically as follows: $U=K F$ where $F=\Sigma f$ and $f=$ tp $U=$ consumptive use in inches, for a given period of time.

$F=$ sum of monthly consumptive use factors.

$K=$ an empirical coefficient, computed from known data for each type of crop.

$f=$ monthly consumptive use factor.

$t=$ mean monthly temperature in degrees Fahrenheit.

$p=$ monthly percent of total daylight hours during the vear.

Consumptive use data for a particular crop from ore area are applicable to other areas by means of the above formula according to Blaney and Criddle if the following assumptions are made: The fertility and producing power of the soils are similar.

Sufficient water is applied and at proper time to maintain good growing con fitions.

The length of growing season, to a large extent, determines the production and annual consumptive use of water for continuous growing crops such as alfalfa and pasture. Consumptive use of water varies directly with the consumptive use factor.

The effects of insects and diseases on the crops are similar from year to year.

The coefficients used in this formula are based primarily upon studies made by tank and field-plot methods in many parts of the West. A list of the $r$ values and lengths of growing season for use in this formula follows:

Tentative values of the consumptive-use coefficient $(K)$

[After Blaney and Criddle]

\begin{tabular}{l|l|r}
\hline \multicolumn{1}{c|}{ Crop } & $\begin{array}{c}\text { Length of growing } \\
\text { season or period }\end{array}$ & $\begin{array}{c}\text { Consumptive use } \\
\text { coefficient }(K)\end{array}$ \\
\hline Alfalfa & Between frosts & 0.85 \\
Beans & 3 months & .65 \\
Corn & 4 months & .75 \\
Cotton & 7 months & .62 \\
Citrus orchard & 7 months & .55 \\
Deciduous orchard & Between frosts & .65 \\
Pasture & Between frosts & .75 \\
Potatoes & 3 months & .70 \\
Small grains & 3 months & .75 \\
Sorghum & 5 months & .70 \\
Sugar beets & $51 / 2$ months & .70 \\
\hline
\end{tabular}


A comparison has been made between the methods of Lowry and Johnson and those of Blaney and Criddle for estimating consumptive use and an actual determination by the inflow-outflow method in the lower Yakima Valley in Washington. The inflow-outflow studies and the computations of Lowry and Johnson were made by the U. S. Bureau of Reclamation in connection with the determination of water requirements for the proposed Kennewick division, an extension of the Yakima Project. The Blaney-Criddle method was applied by the writer to base data extracted from the report by the Bureau of Reclamation. Net consumptive use values were computed for both indirect methods. The net consumptive use as determined by the inflow-outflow studies covering the period 193645 was 1.94 acre-feet per acre. The Lowry-Johnson method gave a value of 2.04 acre-feet per acre, and the Blaney-Criddle method gave 1.97 acre-feet per acre.

The values derived by these two indirect methods agree very closely with the measured values. It is therefore believed that either method, properly used, will yield reliable estimates of net consumptive use for areas where direct measurements are not available.

\section{IRRIGATED AREAS AND ESTIMATED STREAMFLOW DEPLETION}

The tables on the following pages include data on irrigated acreage, annual discharge in acre-feet, and estimated streamflow depletion in acre-feet per year, for many tributary basins of the Columbia River basin above The Dalles, Oreg. The Canadian portion of the basin is not included in these tabulations, but the available information regarding irrigation in Canada is included with the discussion of appropriate subdivisions.

The drainage area above The Dalles, Oreg., was sublivided on basis of available data on irrigation and streamflow as vrell as hydrologic factors. In general these subdivisions were made to conform with the locations of key gaging stations or to span all of the basin of a tributary stream. Areas were kept as small as possible, consistent with available data, in order to provide detailed information on irrigation and depletion intributary stroams. It was not always possible, however, to determine the irrigated acreage within a small basin or restricted area. Reccrds of irrigation of ten were available only by counties or by basins of large tributary streams.

The following list shows the tributary basins, subdivisions, or gaging stations, for which data on irrigated area, or irrigated area, and estimated streamflow depletion, are given. The numbers correspond to locations shown on plate 1 . 


\section{List of tributary basins, subdivisions, or gaging stations for wnich data are given}

1. Kootenai River at Bonners Ferry, Idaho.

2. Clark Fork above Missoula, Mont.

3. Bitterroot River basin.

4. Clark Fork below Missoula, Mont.

5. Clark Fork at St. Regis, Mont.

6. Flathead River basin above Flathead Lake, Mont.

7. Flathead River at mouth.

8. Clark Fork near Plains, Mont.

9. Priest River basin.

10. Pend Oreille River at Priest River, Idaho.

11. Pend Oreille River below Z Canyon near Metaline Falls, Wash.

12. Columbia River at international boundary.

13. Kettle River near Laurier, Wash.

14. Kettle River at mouth.

15. Colville River basin.

16. Spokane River at Post Falls, Idaho.

17. Spokane River at Spokane, Wash.

18. Spokane River at mouth.

19. Columbia River at Grand Coulee Dam, Wash.

20. Similkameen River near Nighthawk, Wash.

21. Okanogan River near Tonasket, Wash.

22. Okanogan River at mouth.

23. Methow River at Twisp, Wash.

24. Methow River at Pateros, Wash.

25. Chelan River basin.

26. Entiat River at Entiat, Wash.

27. Wenatchee River at Peshastin, Wash.

28. Wenatchee River at mouth.

29. Columbia River at Trinidad, Wash.

30. Crab Creek basin.

31. Yakima River at Umtanum, Wash.

32. Yakima River near Parker, Wash.

33. Yakima River at Kiona, Wash.

34. Yakima River basin.

35. Columbia River basin above mouth of Snake River.

36. Snake River basin in Wyoming.

37. Snake River near Heise, Idaho.

38. Henrys Fork near Ashton, Idaho.

39. Falls River near Squirrel, Idaho.

40. Teton River above St. Anthony, Idaho.

41. Snake River near Blackfoot, Idaho.

42. Portneuf River basin.

43. Snake River at Neeley, Idaho.

44. Snake River at Milner, Idaho.

45. Mud Lake basin.

46. Little Lost River basin.

47. Big Lost River above Mackay Reservoir, Idaho.

48. Big Lost River basin.

49. Big Wood River basin.

50. Snake River at King Hill, Idaho.

51. Bruneau River near Hot Spring, Idaho.

52. Bruneau River basin.

53. Snake River near Murphy, Idaho.

54. Owyhee River basin in Nevada.

55. Jordan Creek above Jordan Valley, Oreg.

56. Owyhee River above Owyhee Reservoir, Oreg.

57. Owyhee River at mouth.

58. South Fork Boise River near Featherville, Idaho.

59. South Fork Boise River at Anderson Ranch Dam, Idaho.

60. Boise River at Dowling ranch near Arrowrock, Idaho.

61. Moore Creek near Arrowrock, Idaho.

62. Boise' River basin.

63. Malheur River below Warm Springs Reservoir, Oreg.

64. Malheur River basin.

65. South Fork Payette River near Banks, Idaho.

66. Lake Fork of Payette River below Lake Irrigation District canal, near McCall, Idaho. 
List of tributary basins, subdivisions, or gaging stations for which dat a are given-Continued

67. North Fork Payette River at Cascade, Idaho.

68. North Fork Payette River near Banks, Idaho.

69. Payette River near Horseshoe Bend, Idaho.

70. Payette River at mouth.

71. Weiser River at Starkey, Idaho.

72. Weiser River near Council, Idaho.

73. Wei ser River near Cambridge, Idaho.

74. Little Weiser River near Indian Valley, Idaho.

75. Weiser River above Crane Creek near Weiser, Idaho.

76. Weiser River at mouth.

77. Snake River at Weiser, Idaho.

78. Burnt River near Hereford, Oreg.

79. Burnt River at mouth.

80. Powder River near Robinette, Oreg.

81. Snake River at Oxbow, Oreg.

82. Pine Creek basin.

83. Imnaha River at Imnaha, Oreg.

84. Salmon River near Obsidian, Idaho.

85. Vall ey Creek at Stanley, Idaho.

86. Salmon River below Yankee Fork near Clayton, Idaho.

87. Salmon River near Challis, Idaho.

88. Pahsimeroi River near May, Idaho.

89. North Fork Salmon River at North Fork, Idaho.

90. Salmon River near French Creek, Idaho.

91. Little Salmon River basin.

92. Salmon River at White Bird, Idaho.

93. Wallowa River basin.

94. Grande Ronde River at Rondowa, Oreg.

95. Clearwater River at Kamiah, Idaho.

96. Clearwater River at mouth.

97. Snake River near Clarkston, Wash.

98. Columbia River basin below the Snake River, Wash.

99. Walla Walla River basin.

100. McKay Creek basin.

101. Birch Creek basin.

102. Butter Creek basin.

103. Umatilla River near Umatilla, Oreg.

104. Willow Creek basin.

105. North Fork John Day River at Monument, Oreg.

106. John Day River at McDonald ferry, Oregon.

107. Crooked River near Post, Oreg.

108. Crooked River above Hoffman Dam near Prineville, Oreg.

109. Crooked River near Culver, Oreg.

110. Deschutes River near Madras, Oreg.

111. White River below Tygh Valley, Oreg.

112. Deschutes River at Moody, near Biggs, Oreg.

113. Columbia River near The Dalles, Oreg.

Records of the irrigated area for these tributary basins were then accumulated at downstream points. Thus, where tabulations are given for more than one location on a stream the tabulations for the downstream station include those for the upstream station. The irrigated area between any two stations on the same stream may be determined by subtraction. The data was subjected to a minimum of "rounding off" and often on main stem stations, carried to more significant figures than the overall accuracy may seem to warrant. However this procedure was followed in order to minimize the possible errors in accumulations. Some estimates were necessary, especially for earlier years to formulate the tabulations by subdivisions given in this report. 
Gaging stations are not always in desirable locations with respect to irrigated areas and return flow. The gaging stations chosen are those where depletion estimates would be significant. An ideal location for this purpose would be below all points of diversion, and below points where return flow and wasteways reenter the stream. In general, gaging stations that sulstantially meet these qualifications were selected, and any material deviation is noted. Gaging stations located within an irrigated area, with unmeasured canal flow bypassing those stations and with a complicated or unknown pattern of wasteways and return flow, have of necessity been omitted.

Computations that would be applicable to 23 areas for annual and/or net consumptive use by the Lowry-Johnson method were made available by the U. S. Bureau of Reclamation. These localities were well distributed throughout the drainage basin of the Columbia River above The Dalles, Oreg., and the estimates of net consumptive use for the remaining areas were based upon these computed values. The values of net consumptive use in the following table were compared with available experimental data and were spot checked by the Blaney-Criddle method with consistent results.

Commonly the total consumptive use is computed by applying the factor for net consumptive use to the net cropped arsa and allowing an additional 10 to 15 percent to cover other miscellaneous losses, including deep seepage. However, the net cropped area was not always known, and in this report the net consurptive use was applied to the gross farm area. Since the difference between the gross farm area and the net cropped area usually amounts to 10 to 15 percent, no further allowance was made for the usual miscellaneous losses. 
Net consumptive use, by subdivisions of the Columbia River basin

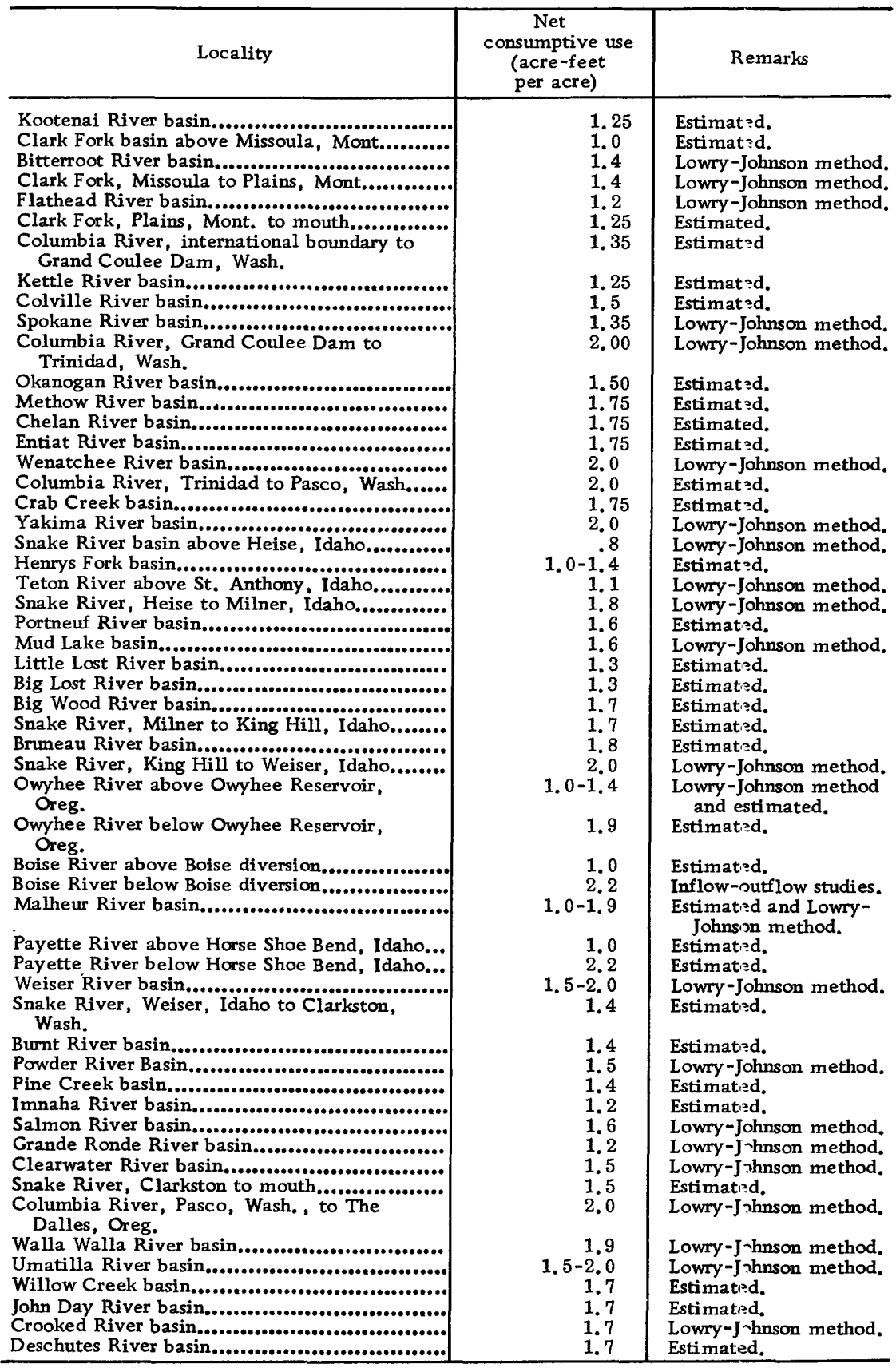


The estimated depletion given in the following tables represents average conditions for the respective subdivisions and assumes an average water supply. The actual depletion in indivic'ual years may be expected to deviate from these values, although in many localities the deviations may be small because of the compensating factors. For example, during years having temperature above normal, the consumptive use might be expected to be grsater than average, but commonly during such years less water is available for irrigation. Some areas often have an inadequate water supply, and the cropped area tends to become adjusted to the available supply. The types of crops undoubtedly influence the consumptive use, but to some extent the types of crops are adjusted to the climate, soil conditions, and the available water supply. These factors tend to promote both regional and year to year diversification in the types of crops grown. Data on irrigation by crop types is very meagre for earlier years, and no attempt has been made to adjust for this factor. Considering all these variables, however, it is believed that the estimated depletions given in following tables are reasonably dependable in terms of average depletion over a period of years.

The estimated depletion, expressed as a percentage of the yield of the watershed, has been computed for many locations in the basin. In this respect the yield was assumed to be the rum of the measured runof $f$ and the estimated depletion. These values were accumulated over a period of years, so as to be representative of average conditions. Where records were available, the 25-year period (1921-45) has been used. In places where the irrigated area has been expanding rapidly, the estimated percentage of depletion for 1946, in terms of the long-term yield has also been computed. It should be reiterated that all of the computations herein are on annual basis and do not take into account the seasonal distribution of water supply or consumptive use.

\section{COLUMBIA RIVER ABOVE THE INTERNATIONAL BOUNDARY}

\section{KOOTEN AI RIVER BASIN}

The Kootenai River (Kootenay in Canada) is primarily a Canadian stream, with 14,500 square miles of the total of 19,300 square miles drainage area lying in southeastern British Columbia. In terms of total drainage area, the Kootenai River is the third largest of the Columbia River tributaries. It is exceeded only by the Snake River and Pend Oreille River.

The United States portion of the basin, lying in northwestern Montana and northern Idaho, is roughly triangular in shape and is largely mountainous. The Galton Range and Salish Mountains form 
the eastern boundary, the Purcell Mountains roughly bisects the basin from north to south, and the Selkirk and Cabinet Mountains form the western boundary. The land devoted to agriculture lies principally in the flood plain of the main stem of the Kootenai River between Bonners Ferry, Idaho, and the internaticnal boundary, and an area along the Tobacco River in Montena. About 50,000 acres in the flood plain north of Bonners Ferry are partially protected by dikes and does not require irrigation because of the high ground-water table underlying the farmed crea.

The Glen Lake Irrigation District is the only sizeable irrigation project in the Kootenai River basin within the boundaries of the United States. It was developed about 1916, and includes about 2,050 acres along the Tobacco River. The remainder of the irrigated land consists of relatively small tracts along Fisher River, in the upper reaches, and near Libby, all in Montana.

Irrigation in the Kootenay River basin in Canada is done on many scattered tracts, which in 1949 totaled 19,700 acres (International Columbia River Engineering Board, 1950, Interim Report on Kootenay River 1933 in the files of the International Joint Commission, Washington and Ottawa).

The following table shows the total irrigated area for the portion of the basin in the United States for the selected years. These data are based on census reports for the years 1890, 1900, 1902, 1919,1929 , and 1939, supplemented by local inquiries. The census report for 1890 shows no irrigation in the basin. For the year 1920, the Montana State Board of Equalization reported a total of 4,780 acres irrigated. Census reports for 1919 show 5,920 acres irrigated. The difference between the two areas probably results from the methods of classification of "irrigated" land.

The net consumptive use was estimated to be 1.25 acre-feet per acre for all irrigated land in this basin. The return flow was estimated to reach the main river south of the international boundary. The average depletion in this basin is insignificant when compared to the flow of the river at Bonners Ferry, Idaho, and has remained essentially constant during the past 20 years. Expressed as a percentage during the period 1929-46, it has amounted to only about 0.1 percent of the yield of this basin. 
Irrigated area in the United States, annual runoff, and estimated depletion for Kootenai River at Bonners Ferry, Idaho

\begin{tabular}{|c|c|c|c|}
\hline Year & $\begin{array}{l}\text { Irrigated area } \\
\text { (acres) }\end{array}$ & $\begin{array}{c}\text { Runoff } \\
\text { (acre-feet) }\end{array}$ & $\begin{array}{l}\text { Estimated } \\
\text { depletion } \\
\text { (acre-feet) }\end{array}$ \\
\hline $\begin{array}{l}1900 \\
1905 \\
1910 \\
1915 \\
1920\end{array}$ & $\begin{array}{l}2,000 \\
3,200 \\
4,200 \\
5,200 \\
6,100\end{array}$ & $\mid$ & $\begin{array}{l}2,500 \\
4,000 \\
5,250 \\
6,500 \\
7,620\end{array}$ \\
\hline $\begin{array}{l}1921 \\
1922 \\
1923 \\
1924 \\
1925\end{array}$ & $\begin{array}{l}6,300 \\
6,450 \\
6,600 \\
6,750 \\
6,950\end{array}$ & 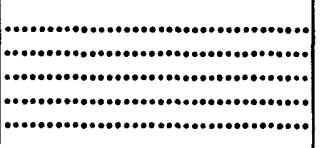 & $\begin{array}{l}7,870 \\
8,060 \\
8,250 \\
8,440 \\
8,680\end{array}$ \\
\hline $\begin{array}{l}1926 \\
1927 \\
1928 \\
1929 \\
1930\end{array}$ & $\begin{array}{l}7,100 \\
7,250 \\
7,400 \\
7,600 \\
7,580\end{array}$ & $\begin{array}{r}8,090,000 \\
8,470,000\end{array}$ & $\begin{array}{l}8,880 \\
9,060 \\
9,250 \\
9,500 \\
9,480\end{array}$ \\
\hline $\begin{array}{l}1931 \\
1932 \\
1933 \\
1934 \\
1935\end{array}$ & $\begin{array}{l}7,560 \\
7,540 \\
7,520 \\
7,500 \\
7,480\end{array}$ & $\begin{array}{r}6,360,000 \\
11,100,000 \\
12,800,000 \\
14,440,000 \\
11,000,000\end{array}$ & $\begin{array}{l}9,450 \\
9,420 \\
9,400 \\
9,380 \\
9,350\end{array}$ \\
\hline $\begin{array}{l}1936 \\
1937 \\
1938 \\
1939 \\
1940\end{array}$ & $\begin{array}{l}7,460 \\
7,440 \\
7,420 \\
7,390 \\
7,400\end{array}$ & $\begin{array}{r}8,082,000 \\
7,499,000 \\
11,590,000 \\
8,367,000 \\
7,771,000\end{array}$ & $\begin{array}{l}9,320 \\
9,300 \\
9,280 \\
9,240 \\
9,250\end{array}$ \\
\hline $\begin{array}{l}1941 \\
1942 \\
1943 \\
1944 \\
1945 \\
1946\end{array}$ & $\begin{array}{l}7,400 \\
7,400 \\
7,400 \\
7,400 \\
7,400 \\
7,400\end{array}$ & $\begin{array}{r}6,508,000 \\
12,190,000 \\
11,910,000 \\
5,599,000 \\
7,729,000 \\
11,970,000\end{array}$ & $\begin{array}{l}9,250 \\
9,250 \\
9,250 \\
9,250 \\
9,250 \\
9,250\end{array}$ \\
\hline
\end{tabular}

\section{PEND OREILLE RIVER BASIN}

The Pend Oreille River basin is largely in western Montana, but it includes a part of the Idaho panhandle, most of Pend Oreille County in northeastern Washington, and two areas totalling 1, 203 square miles in British Columbia. The Flathead River, tributary of the Clark Fork, originates in the eastern area in Eritish Columbia. The western area in Canada is contiguous with the northeast corner of Washington and consists mostly of the drainage area of Salmo River, which joins the Pend Oreille about 14 miles above its mouth. The total drainage area of the Pend Oreille basin is 25,960 square miles, of which 24,757 square miles are in the United States, making it the second largest tributary of the Columbia in terms of drainage area.

The drainage basin is an area of conifer forests, lying on the western slope of the Rocky Mountains. Its perimeter is defined by the divides of various mountain ranges, and numerous other ranges are included within its boundaries. On the east, southeast and south, the Continental Divide forms the boundary. On the west, 
the Bitterroot Range marks both the basin boundary anc the IdahoMontana State line for a distance of 200 miles. Farther west, the boundary is formed by lesser ranges. To the north, the divide between the Kootenai and the Clark Fork is formed by the Selkirk, Cabinet and Salish Mountains.

Along most of the major tributaries and many of the minor tributaries and intermontane valleys and prairies suitable to agriculture. The floors of these valleys range from 2, 000 tc 4, 000 feet above sea level and are suitable for hay, grain, and certain fruit crops. The greater part of the irrigated areas are in basins of Flathead, Bitterroot and Blackfoot Rivers, where some of the earliest irrigation in the Northwest was done.

CLARK FORK ABOVE MISSOULA, MONT.

Most of the irrigated land above this station lies in scattered tracts along the main Clark Fork and its principal tributaries. The majority of these lands were irrigated prior to 1910 by individuals to provide forage crops for stock ranches.

Approximately half of the irrigated land shown in tre following table is in the Blackfoot River basin, with the largest concentration near Nevada Creek reservoir, which was completed in 1938. Of the remaining half of the irrigated area, the largest concentration is along Flint Creek. In 1938 a reservoir on the East Fork of Rock Creek was built to store additional water for lends in this part of the basin.

The return flow from all irrigated land was assumed to have reentered the main stem of the Clark Fork above this gaging station.

The net consumptive use was estimated to be 1.0 acre-foot per acre per year. The percentage of streamflow depletion based upon the 17-year period, 1930 through 1946, amounted to 6.7 percent of the yield of the watershed and has remained essentially constant during the period of gaging-station record at this point. 
Irrigated area, annual runoff, and estimated depletion for Clark Fork above Missoula, Mont.

\begin{tabular}{|c|c|c|c|}
\hline Year & $\begin{array}{l}\text { Irrigated area } \\
\text { (acres) }\end{array}$ & $\begin{array}{c}\text { Runoff } \\
\text { (acre-feet) }\end{array}$ & $\begin{array}{c}\text { Estimated } \\
\text { depletion } \\
\text { (acre-feet) }\end{array}$ \\
\hline $\begin{array}{l}1870 \\
1880 \\
1890 \\
1900\end{array}$ & $\begin{array}{r}6,000 \\
20,000 \\
53,000 \\
102,000\end{array}$ & $\mid$ & $\begin{array}{r}6,000 \\
20,000 \\
53,000 \\
102,000\end{array}$ \\
\hline $\begin{array}{l}1905 \\
1910 \\
1915 \\
1920\end{array}$ & $\begin{array}{l}117,000 \\
119,500 \\
125,000 \\
130,425\end{array}$ & 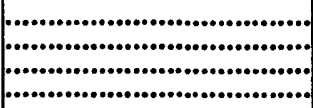 & $\begin{array}{l}117,000 \\
119,500 \\
125,000 \\
130,425\end{array}$ \\
\hline $\begin{array}{l}1921 \\
1922 \\
1923 \\
1924 \\
1925\end{array}$ & $\begin{array}{l}130,415 \\
130,998 \\
130,432 \\
130,164 \\
125,822\end{array}$ & $\mid$ & $\begin{array}{l}130,415 \\
130,998 \\
130,432 \\
130,164 \\
125,822\end{array}$ \\
\hline $\begin{array}{l}1926 \\
1927 \\
1928 \\
1929 \\
1930\end{array}$ & $\begin{array}{l}125,271 \\
124,930 \\
124,690 \\
125,724 \\
124,683\end{array}$ & 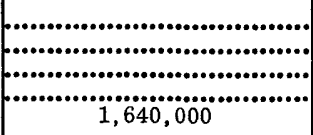 & $\begin{array}{l}125,271 \\
124,930 \\
124,690 \\
125,724 \\
124,683\end{array}$ \\
\hline $\begin{array}{l}1931 \\
1932 \\
1933 \\
1934 \\
1935\end{array}$ & $\begin{array}{l}123,064 \\
128,205 \\
124,745 \\
128,008 \\
127,107\end{array}$ & $\begin{array}{l}1,039,000 \\
1,602,000 \\
2,087,000 \\
2,425,000 \\
1,410,000\end{array}$ & $\begin{array}{l}123,064 \\
128,205 \\
124,745 \\
128,008 \\
127,107\end{array}$ \\
\hline $\begin{array}{l}1936 \\
1937 \\
1938 \\
1939 \\
1940\end{array}$ & $\begin{array}{l}119,987 \\
127,698 \\
120,458 \\
118,576 \\
119,272\end{array}$ & $\begin{array}{l}1,622,000 \\
1,035,000 \\
2,006,000 \\
1,592,000 \\
1,223,000\end{array}$ & $\begin{array}{l}119,987 \\
127,698 \\
120,458 \\
118,576 \\
119,272\end{array}$ \\
\hline $\begin{array}{l}1941 \\
1942 \\
1943 \\
1944 \\
1945 \\
1946\end{array}$ & $\begin{array}{l}118,992 \\
119,327 \\
119,743 \\
119,287 \\
118,720 \\
119,720\end{array}$ & $\begin{array}{r}973,200 \\
1,932,000 \\
2,990,000 \\
1,475,000 \\
1,600,000 \\
1,728,000\end{array}$ & $\begin{array}{l}118,992 \\
119,327 \\
119,743 \\
119,287 \\
118,720 \\
119,720\end{array}$ \\
\hline
\end{tabular}

CLARK FORK BELOW MISSOULA, MONT.

The Bitterroot River basin has at present about 113, 800 acres under irrigation, although the water supply during dry years is inadequate. Very little information is available regarding irrigation prior to 1890 , although a note in the 1889 census states that the Bitterroot Valley was at that time extensively irrigated along the river. Most of the water used for irrigation is supplied by small irrigation companies and districts. The largest organization is the Bitterroot River Irrigation District, which controls approximately 19,000 acres. The return flow from these lands is believed to enter the Bitterroot River above its mouth. The irrigated area and estimated annual depletion are shown in the following table. The net consumptive use of 1.4 acre-feet per acre used to compute the estimated depletion was based upon computations by the Lowry-Johnson method. Assuming the difference between the gaging stations on Clark Fork above and below Missoula to be the yield of the Bitterroot River basin, the percentage depletion was estimated to be 9.6 percent. 
Between the gaging stations on Clark Fork above and below Missoula are three principal diversions from the Clark Fork main stem: Flynn Ditch, Grass Valley Ditch, and Orchard Homes District canal. Flynn Ditch was built about 1906 to provide supplemental water to land which formerly received only flcod water from Granite Creek. This ditch diverts water from the north bank of the Clark Fork at the west edge of Missoula, and serves 1,900 acres. The Grass Valley Ditch diverts water from the Clark Fork above the mouth of the Bitterroot River. Diversion first began in 1903, and at present about 3,000 acres are served. The Orchard Homes District serves two tracts: Tract 1, started alcout 1878 now serves 3,200 acres, the majority of which was ceveloped prior to 1920. Tract 2 includes about 550 acres and was started in 1896. The irrigated area and estimated depletion for the basin above the gaging station on Clark Fork below Missoula, Mont., are shown in the table below. The percentage depletior for this station is 8.4 percent of the yield of the watershed for period 1930 through 1946.

Irrigated area and estimated depletion for Bitterroot River basin

\begin{tabular}{|c|c|c|}
\hline Year & $\begin{array}{l}\text { Irrigated area } \\
\text { (acres) }\end{array}$ & $\begin{array}{c}\text { Estimated } \\
\text { depletion } \\
\text { (acre-feet) }\end{array}$ \\
\hline $\begin{array}{l}1870 \\
1880 \\
1890 \\
1900\end{array}$ & $\begin{array}{r}2,000 \\
5,000 \\
17,000 \\
72,700\end{array}$ & $\begin{array}{r}2,800 \\
7,000 \\
23,800 \\
101,800\end{array}$ \\
\hline $\begin{array}{l}1905 \\
1910 \\
1915 \\
1920\end{array}$ & $\begin{array}{l}101,000 \\
103,000 \\
105,000 \\
107,522\end{array}$ & $\begin{array}{l}141,400 \\
144,200 \\
147,000 \\
150,500\end{array}$ \\
\hline $\begin{array}{l}1921 \\
1922 \\
1923 \\
1924 \\
1925\end{array}$ & $\begin{array}{l}107,522 \\
117,957 \\
115,577 \\
114,028 \\
110,983\end{array}$ & $\begin{array}{l}150,500 \\
165,100 \\
161,800 \\
159,600 \\
155,400\end{array}$ \\
\hline $\begin{array}{l}1926 \\
1927 \\
1928 \\
1929 \\
1930\end{array}$ & $\begin{array}{l}108,868 \\
107,725 \\
107,944 \\
107,951 \\
105,849\end{array}$ & $\begin{array}{l}152,400 \\
150,800 \\
151,100 \\
151,100 \\
148,200\end{array}$ \\
\hline $\begin{array}{l}1931 \\
1932 \\
1933 \\
1934 \\
1935\end{array}$ & $\begin{array}{l}104,957 \\
103,794 \\
103,009 \\
105,628 \\
105,534\end{array}$ & $\begin{array}{l}146,900 \\
145,300 \\
144,200 \\
147,900 \\
147,700\end{array}$ \\
\hline $\begin{array}{l}1936 \\
1937 \\
1938 \\
1939 \\
1940\end{array}$ & $\begin{array}{l}104,290 \\
104,577 \\
104,126 \\
106,420 \\
103,380\end{array}$ & $\begin{array}{l}146,000 \\
146,400 \\
145,800 \\
149,000 \\
144,700\end{array}$ \\
\hline $\begin{array}{l}1941 \\
1942 \\
1943 \\
1944 \\
1945 \\
1946\end{array}$ & $\begin{array}{l}103,062 \\
102,740 \\
102,412 \\
103,222 \\
103,445 \\
105,620\end{array}$ & $\begin{array}{l}144,300 \\
143,800 \\
143,400 \\
144,500 \\
144,800 \\
147,900\end{array}$ \\
\hline
\end{tabular}


Irrigated area, annual runoff, and estimated depletion for Clark Fork below Missoula, Mont.

\begin{tabular}{|c|c|c|c|}
\hline Year & $\begin{array}{l}\text { Irrigated area } \\
\text { (acres) }\end{array}$ & $\begin{array}{c}\text { Runoff } \\
\text { (acre-feet) }\end{array}$ & $\begin{array}{c}\text { Estimated } \\
\text { depletion } \\
\text { (acre-feet) }\end{array}$ \\
\hline $\begin{array}{l}1860 \\
1870 \\
1880 \\
1890 \\
1900\end{array}$ & $\begin{array}{r}1,000 \\
8,000 \\
25,000 \\
70,000 \\
175,000\end{array}$ & 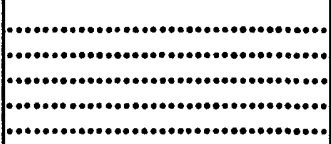 & $\begin{array}{r}1,400 \\
8,800 \\
27,000 \\
76,800 \\
204,000\end{array}$ \\
\hline $\begin{array}{l}1905 \\
1910 \\
1915 \\
1920\end{array}$ & $\begin{array}{l}219,000 \\
223,500 \\
234,600 \\
245,585\end{array}$ & 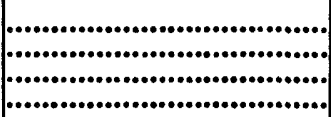 & $\begin{array}{l}259,800 \\
265,100 \\
278,400 \\
288,500\end{array}$ \\
\hline $\begin{array}{l}1921 \\
1922 \\
1923 \\
1924 \\
1925\end{array}$ & $\begin{array}{l}246,075 \\
257,093 \\
254,147 \\
252,330 \\
244,943\end{array}$ & $\mid$ & $\begin{array}{l}289,000 \\
307,500 \\
303,600 \\
301,200 \\
292,600\end{array}$ \\
\hline $\begin{array}{l}1926 \\
1927 \\
1928 \\
1929 \\
1930\end{array}$ & $\begin{array}{l}242,777 \\
241,293 \\
241,272 \\
242,813 \\
239,900\end{array}$ & 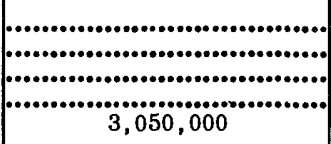 & $\begin{array}{l}289,100 \\
287,100 \\
287,200 \\
289,600 \\
288,800\end{array}$ \\
\hline $\begin{array}{l}1931 \\
1932 \\
1933 \\
1934 \\
1935\end{array}$ & $\begin{array}{l}237,389 \\
241,367 \\
237,122 \\
243,004 \\
242,009\end{array}$ & $\begin{array}{l}1,980,000 \\
3,080,000 \\
3,740,000 \\
4,253,000 \\
2,671,000\end{array}$ & $\begin{array}{l}283,100 \\
286,600 \\
282,000 \\
289,000 \\
287,900\end{array}$ \\
\hline $\begin{array}{l}1936 \\
1937 \\
1938 \\
1939 \\
1940\end{array}$ & $\begin{array}{l}233,645 \\
241,643 \\
233,952 \\
234,364 \\
232,020\end{array}$ & $\begin{array}{l}3,105,000 \\
1,869,000 \\
3,619,000 \\
3,022,000 \\
2,227,000\end{array}$ & $\begin{array}{l}279,100 \\
287,200 \\
279,400 \\
280,700 \\
277,100\end{array}$ \\
\hline $\begin{array}{l}1941 \\
1942 \\
1943 \\
1944 \\
1945 \\
1946\end{array}$ & $\begin{array}{l}231,422 \\
231,425 \\
231,523 \\
231,877 \\
231,533 \\
234,708\end{array}$ & $\begin{array}{l}1,881,000 \\
3,634,000 \\
5,544,000 \\
2,570,000 \\
2,803,000 \\
3,235,000\end{array}$ & $\begin{array}{l}276,400 \\
276,200 \\
276,200 \\
276,900 \\
276,600 \\
280,700\end{array}$ \\
\hline
\end{tabular}

\section{CLARK FORK AT ST. REGIS, MONT.}

The majority of irrigated lands between Missoula anc the gaging station on the Clark Fork at St. Regis, Mont., are scattered in small tracts along tributaries of the main river. The Frenchtown project is the largestirrigation enterprise within this rsach. This project extends from Frenchtown to Huson, Mont. ard includes about 2, 200 acres, all on the north side of the river. The return flow from all irrigated land in this subdivision is assumed to reach the Clark Fork above the gaging station. The irrigated area above this gaging station, and the estimated depletion are given in the following table. Based upon the records for the period 1921 through 1945 and a net consumptive use for this subdivision of 1.4 acrefeet per acre, the percentage depletion of the yield of the watershed was estimated to be 3.3 percent. 
Irrigated area, annual runoff, and estimated depletion for Clark Fork at St. Regis, Mont.

\begin{tabular}{|c|c|c|c|}
\hline Year & $\begin{array}{l}\text { Irrigated area } \\
\text { (acres) }\end{array}$ & $\begin{array}{c}\text { Runoff } \\
\text { (acre-feet) }\end{array}$ & $\begin{array}{l}\text { Estimated } \\
\text { depletion } \\
\text { (acre-feet) }\end{array}$ \\
\hline $\begin{array}{l}1860 \\
1870 \\
1880 \\
1890 \\
1900\end{array}$ & $\begin{array}{r}1,000 \\
8,000 \\
25,000 \\
74,000 \\
180,000\end{array}$ & $\mid$ & $\begin{array}{r}1,400 \\
8,800 \\
27,000 \\
76,800 \\
211,200\end{array}$ \\
\hline $\begin{array}{l}1905 \\
1910\end{array}$ & $\begin{array}{l}232,000 \\
243,100\end{array}$ & {$[\ldots \ldots \ldots \ldots \ldots \ldots \ldots \ldots \ldots \ldots \ldots \ldots \ldots \ldots \ldots \ldots \ldots \ldots \ldots \ldots \ldots \ldots \ldots$} & $\begin{array}{l}278,000 \\
292,400\end{array}$ \\
\hline $\begin{array}{l}1911 \\
1912 \\
1913 \\
1914 \\
1915\end{array}$ & 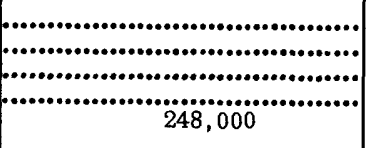 & $\begin{array}{l}5,630,000 \\
6,550,000 \\
7,760,000 \\
5,230,000 \\
4,220,000\end{array}$ & 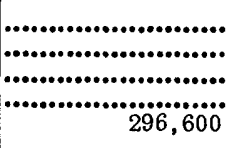 \\
\hline $\begin{array}{l}1916 \\
1917 \\
1918 \\
1919 \\
1920\end{array}$ & 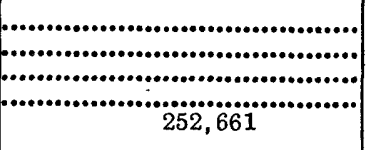 & $\begin{array}{l}8,460,000 \\
8,090,000 \\
7,840,000 \\
3,930,000 \\
5,140,000\end{array}$ & 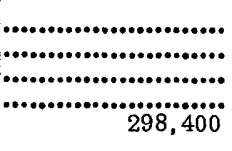 \\
\hline $\begin{array}{l}1921 \\
1922 \\
1923 \\
1924 \\
1925\end{array}$ & $\begin{array}{l}253,155 \\
264,287 \\
261,392 \\
259,595 \\
252,136\end{array}$ & $\begin{array}{r}6,270,000 \\
5,840,000 \\
5,260,000 \\
* 3,524,000 \\
+5,811,000\end{array}$ & $\begin{array}{l}298,900 \\
317,600 \\
313,700 \\
311,400 \\
302,700\end{array}$ \\
\hline $\begin{array}{l}1926 \\
1927 \\
1928 \\
1929 \\
1930\end{array}$ & $\begin{array}{l}249,995 \\
248,519 \\
248,448 \\
249,980 \\
247,094\end{array}$ & $\begin{array}{r}2,932,000 \\
* 6,414,000 \\
* 7,250,000 \\
* 3,721,000 \\
3,930,000\end{array}$ & $\begin{array}{l}299,200 \\
297,200 \\
297,200 \\
299,600 \\
298,900\end{array}$ \\
\hline $\begin{array}{l}1931 \\
1932 \\
1933 \\
1934 \\
1935\end{array}$ & $\begin{array}{l}244,577 \\
248,580 \\
244,363 \\
250,160 \\
249,163\end{array}$ & $\begin{array}{l}2,590,000 \\
4,330,000 \\
5,400,000 \\
6,780,000 \\
3,988,000\end{array}$ & $\begin{array}{l}293,200 \\
296,700 \\
293,100 \\
299,000 \\
297,000\end{array}$ \\
\hline $\begin{array}{l}1936 \\
1937 \\
1938 \\
1939 \\
1940\end{array}$ & $\begin{array}{l}240,734 \\
250,918 \\
243,229 \\
243,625 \\
241,254\end{array}$ & $\begin{array}{l}4,139,000 \\
2,647,000 \\
4,856,000 \\
3,886,000 \\
3,027,000\end{array}$ & $\begin{array}{l}289,000 \\
300,200 \\
292,400 \\
293,700 \\
290,000\end{array}$ \\
\hline $\begin{array}{l}1941 \\
1942 \\
1943 \\
1944 \\
1945 \\
1946\end{array}$ & $\begin{array}{l}240,738 \\
240,751 \\
240,838 \\
241,181 \\
240,971 \\
243,979\end{array}$ & $\begin{array}{l}2,476,000 \\
4,697,000 \\
7,307,000 \\
3,246,000 \\
3,767,000 \\
4,282,000\end{array}$ & $\begin{array}{l}289,400 \\
289,200 \\
289,200 \\
289,900 \\
289,800 \\
293,700\end{array}$ \\
\hline
\end{tabular}

* Estimated, or partly estimated in unpublished report. 


\section{FL ATHEAD RIVER}

In the Flathead River basin, irrigation above Flathead Lake has been developed principally by private enterprise, tre largest project being the Ashley Irrigation District. The remainder of the irrigated area lies in small tracts along Swan River, Whitefish River, and Stillwater River. A relatively small amount of land has been developed within the proposed Kalispell Project of the U. S. Bureau of Reclamation by private enterprise.

Below Flathead Lake, the largest development has been the Flathead Project, initiated by the U. S. Bureau of Reclemation in 1907 and delegated to the Bureau of Indian Affairs in 1924 for completion and operation. All the land lies within the early boundaries of the Flathead Indian Reservation and the project is designed for the ultimate irrigation of 138,000 acres in three divisions: Camas, Jocko, and Mission Valley. The project area of Camas division consists of about 13,000 acres primarily in the Little Bitterroot River basin, which is served by gravity diversion from the main stream and tributaries. This water supply has been augmented by two small diversions from the Thompson River basin. Diversion from Alder Creek began about 1933 and from McGinnis Creek in 1941. The Jocko division includes about 13,400 acres and is served by gravity diversion from Jocko River and Finley Creek. A diversion from Placid Creek, an indirsct tributary of the Blackfoot River, enters the Middle Fork of the Jocko River but is generally used for irrigation in the Mission Valley division. The major sources of water supply for the Mission Valley division are the minor tributaries of the Flathead River arising in the Mission Range and water pumped directly from the Flathead River near the lake outlet. About 113,700 acres nay eventually be irrigated in this division. There is about 148,000 acrefeet of storage in all three divisions, and to a large extent all of the divisions are interconnected. The return flow from all these lands is assumed to enter the Flathead River above its mouth. The net consumptive use for all lands in the entire basin was estimated to be 1.2 acre-feet per acre and was based upon computations by the Lowry-Johnson method. The irrigated area and estimated depletion for the Flathead River basin, disregerding the minor effects of importation of water from adjacent basins, are shown in the following table. 
Irrigated area and estimated depletion for Flathead River basin, Montana

\begin{tabular}{|c|c|c|c|}
\hline \multirow[b]{2}{*}{ Year } & \multirow{2}{*}{$\begin{array}{c}\text { Flathead River above } \\
\text { Flathead Lake } \\
\begin{array}{c}\text { Irrigated area } \\
\text { (acres) }\end{array}\end{array}$} & \multicolumn{2}{|c|}{ Flathead River at nouth } \\
\hline & & $\begin{array}{l}\text { Irrigated area } \\
\text { (acres) }\end{array}$ & $\begin{array}{c}\text { Estimated } \\
\text { depletion } \\
\text { (acre-feet) }\end{array}$ \\
\hline 1900 & 1,000 & 6,070 & 7,280 \\
\hline $\begin{array}{l}1905 \\
1910 \\
1915 \\
1920\end{array}$ & $\begin{array}{l}2,000 \\
3,100 \\
3,300 \\
3,501\end{array}$ & $\begin{array}{l}11,500 \\
14,500 \\
19,700 \\
38,367\end{array}$ & $\begin{array}{l}13,800 \\
17,400 \\
23,640 \\
46,040\end{array}$ \\
\hline $\begin{array}{l}1921 \\
1922 \\
1923 \\
1924 \\
1925\end{array}$ & $\begin{array}{l}3,501 \\
3,501 \\
3,501 \\
3,501 \\
3,501\end{array}$ & $\begin{array}{l}36,016 \\
35,888 \\
24,026 \\
35,371 \\
38,018\end{array}$ & $\begin{array}{l}43,220 \\
43,070 \\
28,830 \\
42,440 \\
45,620\end{array}$ \\
\hline $\begin{array}{l}1926 \\
1927 \\
1928 \\
1929 \\
1930\end{array}$ & $\begin{array}{l}3,401 \\
3,401 \\
3,301 \\
3,301 \\
3,201\end{array}$ & $\begin{array}{l}39,378 \\
31,568 \\
39,831 \\
45,331 \\
48,831\end{array}$ & $\begin{array}{l}47,250 \\
37,880 \\
47,260 \\
54,400 \\
58,600\end{array}$ \\
\hline $\begin{array}{l}1931 \\
1932 \\
1933 \\
1934 \\
1935\end{array}$ & $\begin{array}{l}3,201 \\
3,161 \\
3,399 \\
3,301 \\
3,301\end{array}$ & $\begin{array}{l}57,631 \\
62,891 \\
64,429 \\
65,531 \\
72,331\end{array}$ & $\begin{array}{l}69,160 \\
75,470 \\
77,310 \\
78,640 \\
86,800\end{array}$ \\
\hline $\begin{array}{l}1936 \\
1937 \\
1938 \\
1939 \\
1940\end{array}$ & $\begin{array}{l}3,301 \\
3,230 \\
3,278 \\
2,240 \\
2,562\end{array}$ & $\begin{array}{l}73,331 \\
77,760 \\
81,308 \\
80,570 \\
81,892\end{array}$ & $\begin{array}{l}88,000 \\
93,310 \\
97,570 \\
96,680 \\
98,270\end{array}$ \\
\hline $\begin{array}{l}1941 \\
1942 \\
1943 \\
1944 \\
1945 \\
1946\end{array}$ & $\begin{array}{l}2,001 \\
2,008 \\
2,721 \\
2,713 \\
2,701 \\
2,701\end{array}$ & $\begin{array}{l}80,931 \\
76,438 \\
83,151 \\
85,343 \\
88,431 \\
90,731\end{array}$ & $\begin{array}{r}97,120 \\
91,720 \\
99,780 \\
102,410 \\
106,120 \\
108,880 \\
\end{array}$ \\
\hline
\end{tabular}

PEND OREILLE RIVER BELOW Z CANYON NEAR METALINE FALLS, WASH.

Irrigation below the mouth of Flathead River lies in scattered small tracts along the Clark Fork and Pend Oreille Rivers. An irrigation district near Usk, Wash. utilizes water from Calispell Creek to irrigate about 1,500 acres. The net consumptive use for this subdivision of the basin was estimated to be 1.25 acre-feet per acre. All return flow was assumed to have reentered the main river above the indicated gages. The irrigated area, runoff, and estimated depletion at the gaging station on Clark Fork n?ar Plains, Mont. are shown in the following table. For the period 1921 through 1945 the percent depletion was estimated to be 2.7 percent. The irrigated area for Priest River and Pend Oreille River at Priest River, Idaho is shown in the table on page 34 . The total irrigated area, runoff, and estimated depletion, in the basin above the gaging station on Pend Oreille River below $Z$ Canyon near Metaline Falls, Wash., is shown in the table on page 35 . 
Irrigated area, annual runoff, and estimated depletion for Clark Fork near Plains, Mont.

\begin{tabular}{|c|c|c|c|}
\hline Year & $\begin{array}{l}\text { Irrigated area } \\
\text { (acres) }\end{array}$ & $\begin{array}{c}\text { Runoff } \\
\text { (acre-feet) }\end{array}$ & $\begin{array}{l}\text { Estimated } \\
\text { depletion } \\
\text { (acre-feet) }\end{array}$ \\
\hline $\begin{array}{l}1860 \\
1870 \\
1880 \\
1890 \\
1900\end{array}$ & $\begin{array}{r}1,000 \\
8,000 \\
25,000 \\
74,000 \\
186,700\end{array}$ & 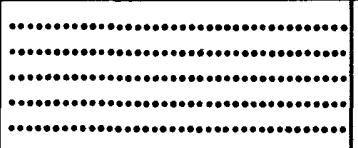 & $\begin{array}{r}1,400 \\
8,800 \\
27,000 \\
76,800 \\
218,500\end{array}$ \\
\hline $\begin{array}{l}1905 \\
1910\end{array}$ & $\begin{array}{l}243,700 \\
257,800\end{array}$ & 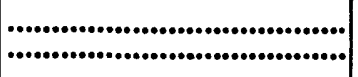 & $\begin{array}{l}291,800 \\
309,800\end{array}$ \\
\hline $\begin{array}{l}1913 \\
1914 \\
1915\end{array}$ & 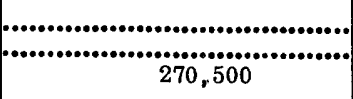 & $\begin{array}{l}17,400,000 \\
12,500,000 \\
11,400,000\end{array}$ & 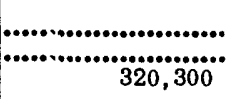 \\
\hline $\begin{array}{l}1916 \\
1917 \\
1918 \\
1919 \\
1920\end{array}$ & 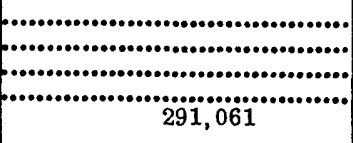 & $\begin{array}{r}* 20,271,000 \\
* 17,601,000 \\
17,400,000 \\
10,900,000 \\
* 12,552,000\end{array}$ & 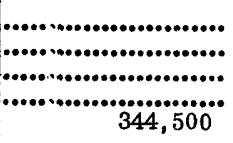 \\
\hline $\begin{array}{l}1921 \\
1922 \\
1923 \\
1924 \\
1925\end{array}$ & $\begin{array}{l}289,204 \\
300,208 \\
285,451 \\
294,999 \\
290,187\end{array}$ & $\begin{array}{r}16,500,000 \\
14,100,000 \\
14,100,000 \\
11,600,000 \\
17,617,000\end{array}$ & $\begin{array}{l}342,200 \\
360,700 \\
342,600 \\
353,900 \\
348,400\end{array}$ \\
\hline $\begin{array}{l}1926 \\
1927 \\
1928 \\
1929 \\
1930\end{array}$ & $\begin{array}{l}289,406 \\
280,120 \\
288,312 \\
295,344 \\
295,958\end{array}$ & $\begin{array}{r}9,150,000 \\
19,300,000 \\
21,300,000 \\
11,000,000 \\
10,100,000\end{array}$ & $\begin{array}{l}346,500 \\
335,100 \\
344,500 \\
354,000 \\
357,500\end{array}$ \\
\hline $\begin{array}{l}1931 \\
1932 \\
1933 \\
1934 \\
1935\end{array}$ & $\begin{array}{l}302,241 \\
311,504 \\
308,825 \\
315,724 \\
321,527\end{array}$ & $\begin{array}{r}8,250,000 \\
13,700,000 \\
16,000,000 \\
18,430,000 \\
12,660,000\end{array}$ & $\begin{array}{l}362,400 \\
372,200 \\
370,400 \\
377,700 \\
383,800\end{array}$ \\
\hline $\begin{array}{l}1936 \\
1937 \\
1938 \\
1939 \\
1940\end{array}$ & $\begin{array}{l}314,098 \\
328,711 \\
324,570 \\
324,228 \\
323,179\end{array}$ & $\begin{array}{r}11,670,000 \\
8,784,000 \\
12,250,000 \\
11,460,000 \\
8,213,000\end{array}$ & $\begin{array}{l}377,000 \\
393,600 \\
390,000 \\
390,300 \\
388,300\end{array}$ \\
\hline $\begin{array}{l}1941 \\
1942 \\
1943 \\
1944 \\
1945 \\
1946\end{array}$ & $\begin{array}{l}321,702 \\
317,222 \\
324,022 \\
326,557 \\
329,435 \\
334,743\end{array}$ & $\begin{array}{r}6,404,000 \\
12,740,000 \\
17,710,000 \\
7,862,000 \\
10,330,000 \\
13,480,000\end{array}$ & $\begin{array}{l}386,600 \\
381,000 \\
389,000 \\
392,400 \\
396,000 \\
402,600\end{array}$ \\
\hline
\end{tabular}

- Estimated, or partly estimated in unpublished report. 
Irrigated area in acres for indicated years

\begin{tabular}{|c|c|c|}
\hline Year & Priest River basin & $\begin{array}{l}\text { Pend Oreille River } \\
\text { at Prie-t. River, Idaho }\end{array}$ \\
\hline $\begin{array}{l}1860 \\
1870 \\
1880 \\
1890 \\
1900\end{array}$ & 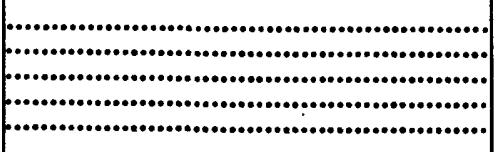 & $\begin{array}{r}1,000 \\
8,000 \\
25,000 \\
74,000 \\
186,100\end{array}$ \\
\hline $\begin{array}{l}1905 \\
1910 \\
1915 \\
1920\end{array}$ & - & $\begin{array}{l}245,400 \\
260,900 \\
273,000 \\
293,749\end{array}$ \\
\hline $\begin{array}{l}1921 \\
1922 \\
1923 \\
1924 \\
1925\end{array}$ & $\begin{array}{l}63 \\
63 \\
63 \\
63 \\
63\end{array}$ & $\begin{array}{l}291,894 \\
302,925 \\
288,170 \\
297,721 \\
292,912\end{array}$ \\
\hline $\begin{array}{l}1926 \\
1927 \\
1928 \\
1929 \\
1930\end{array}$ & $\begin{array}{l}63 \\
73 \\
73 \\
73 \\
73\end{array}$ & $\begin{array}{l}292,150 \\
282,892 \\
291,106 \\
298,141 \\
298,758\end{array}$ \\
\hline $\begin{array}{l}1931 \\
1932 \\
1933 \\
1934 \\
1935\end{array}$ & $\begin{array}{l}73 \\
73 \\
73 \\
73 \\
73\end{array}$ & $\begin{array}{l}305,044 \\
314,309 \\
311,632 \\
318,534 \\
324,360\end{array}$ \\
\hline $\begin{array}{l}1936 \\
1937 \\
1938 \\
1939 \\
1940\end{array}$ & $\begin{array}{l}73 \\
73 \\
73 \\
73 \\
73\end{array}$ & $\begin{array}{l}316,934 \\
331,549 \\
327,410 \\
327,071 \\
326,025\end{array}$ \\
\hline $\begin{array}{l}1941 \\
1942 \\
1943 \\
1944 \\
1945 \\
1946\end{array}$ & $\begin{array}{l}73 \\
73 \\
73 \\
73 \\
73 \\
73 \\
\end{array}$ & $\begin{array}{l}324,531 \\
320,083 \\
326,885 \\
329,423 \\
332,304 \\
337,650 \\
\end{array}$ \\
\hline
\end{tabular}


Irrigated area, annual runoff, and estimated depletion for Pend Oreille River below $Z$ Canyon near Metaline Falls, Wash.

\begin{tabular}{|c|c|c|c|}
\hline Year & $\begin{array}{l}\text { Irrigated area } \\
\text { (acres) }\end{array}$ & $\begin{array}{c}\text { Rumoff } \\
\text { (acre-feet) }\end{array}$ & $\begin{array}{l}\text { Estimated } \\
\text { depletion } \\
\text { (acre-feet) }\end{array}$ \\
\hline $\begin{array}{l}1860 \\
1870 \\
1880 \\
1890 \\
1900\end{array}$ & $\begin{array}{r}1,000 \\
8,000 \\
25,000 \\
74,000 \\
186,213\end{array}$ & 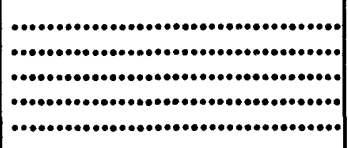 & $\begin{array}{r}1,400 \\
8,800 \\
27,000 \\
76,800 \\
218,600\end{array}$ \\
\hline $\begin{array}{l}1905 \\
1910\end{array}$ & $\begin{array}{l}245,416 \\
260,932\end{array}$ & 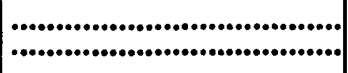 & $\begin{array}{l}293,900 \\
316,700\end{array}$ \\
\hline $\begin{array}{l}1913 \\
1914 \\
1915\end{array}$ & ( & $\begin{array}{l}24,800,000 \\
17,600,000 \\
15,400,000\end{array}$ & $\cdots \cdots \cdots \cdots \cdots \cdots \cdots \cdots \cdots$ \\
\hline $\begin{array}{l}1916 \\
1917 \\
1918 \\
1919 \\
1920\end{array}$ & 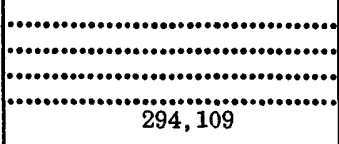 & $\begin{array}{l}27,900,000 \\
23,300,000 \\
21,800,000 \\
15,800,000 \\
15,700,000\end{array}$ & 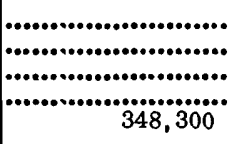 \\
\hline $\begin{array}{l}1921 \\
1922 \\
1923 \\
1924 \\
1925\end{array}$ & $\begin{array}{l}292,314 \\
303,405 \\
288,710 \\
297,721 \\
293,672\end{array}$ & $\begin{array}{l}22,400,000 \\
17,800,000 \\
18,100,000 \\
14,500,000 \\
23,200,000\end{array}$ & $\begin{array}{l}346,100 \\
364,700 \\
346,700 \\
357,300 \\
352,800\end{array}$ \\
\hline $\begin{array}{l}1926 \\
1927 \\
1928 \\
1929 \\
1930\end{array}$ & $\begin{array}{l}292,870 \\
283,672 \\
291,946 \\
299,041 \\
299,733\end{array}$ & $\begin{array}{l}11,700,000 \\
25,300,000 \\
27,600,000 \\
13,900,000 \\
12,600,000\end{array}$ & $\begin{array}{l}350,800 \\
339,500 \\
349,000 \\
358,600 \\
362,100\end{array}$ \\
\hline $\begin{array}{l}1931 \\
1932 \\
1933 \\
1934 \\
1935\end{array}$ & $\begin{array}{l}306,094 \\
315,434 \\
312,832 \\
319,709 \\
325,710\end{array}$ & $\begin{array}{l}11,100,000 \\
19,500,000 \\
22,400,000 \\
26,170,000 \\
18,270,000\end{array}$ & $\begin{array}{l}367,200 \\
377,100 \\
375,400 \\
382,700 \\
389,000\end{array}$ \\
\hline $\begin{array}{l}1936 \\
1937 \\
1938 \\
1939 \\
1940\end{array}$ & $\begin{array}{l}317,469 \\
333,049 \\
328,985 \\
328,721 \\
327,675\end{array}$ & $\begin{array}{l}15,560,000 \\
12,130,000 \\
18,410,000 \\
15,250,000 \\
12,270,000\end{array}$ & $\begin{array}{l}381,200 \\
399,100 \\
395,500 \\
395,600 \\
393,900\end{array}$ \\
\hline $\begin{array}{l}1941 \\
1942 \\
1943 \\
1944 \\
1945 \\
1946\end{array}$ & $\begin{array}{l}326,181 \\
321,733 \\
328,535 \\
331,173 \\
333,954 \\
339,300\end{array}$ & $\begin{array}{r}9,951,000 \\
18,170,000 \\
23,760,000 \\
10,480,000 \\
14,680,000 \\
19,670,000\end{array}$ & $\begin{array}{l}391,200 \\
386,600 \\
394,600 \\
398,200 \\
401,600 \\
408,300\end{array}$ \\
\hline
\end{tabular}

\section{COLUMBIA RIVER AT INTERNATIONAL BOUNDARY}

The following table shows the irrigated area, runoff, and estimated depletion for the basin above the gaging station on Columbia River at the international boundary. This is essentially a summation of the Kootenai and Pend Oreille River basins. No allowance has been made for the irrigated area in Canada, which on basis of published reports is assumed to be less than 25,000 acres. The average net depletion based upon the period 1939 through 1946 was estimated at 0.7 percent of the yield of the watershed above this point. 
Irrigated area, annual runoff, and estimated depletion for Columbia River at the international boundary

\begin{tabular}{|c|c|c|c|}
\hline Year & $\begin{array}{c}\text { Irrigated area } \\
\text { (acres) }\end{array}$ & $\begin{array}{c}\text { Runoff } \\
\text { (acre-feet) }\end{array}$ & $\begin{array}{c}\text { Estimated } \\
\text { depletion } \\
\text { (acre-feet) }\end{array}$ \\
\hline $\begin{array}{l}1860 \\
1870 \\
1880 \\
1890 \\
1900\end{array}$ & $\begin{array}{r}1,000 \\
8,000 \\
25,000 \\
74,000 \\
188,213\end{array}$ & 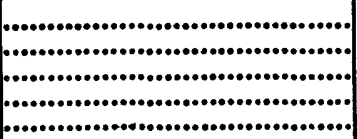 & $\begin{array}{r}1,400 \\
8,800 \\
27,000 \\
76,800 \\
221,100\end{array}$ \\
\hline $\begin{array}{l}1905 \\
1910 \\
1915 \\
1920\end{array}$ & $\begin{array}{l}248,616 \\
265,132 \\
278,242 \\
300,209\end{array}$ & 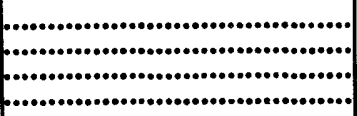 & $\begin{array}{l}297,400 \\
321,900 \\
341,900 \\
355,900\end{array}$ \\
\hline $\begin{array}{l}1921 \\
1922 \\
1923 \\
1924 \\
1925\end{array}$ & $\begin{array}{l}298,614 \\
309,855 \\
295,310 \\
305,071 \\
300,522\end{array}$ & - & $\begin{array}{l}354,000 \\
372,800 \\
354,900 \\
365,700 \\
361,500\end{array}$ \\
\hline $\begin{array}{l}1926 \\
1927 \\
1928 \\
1929 \\
1930\end{array}$ & $\begin{array}{l}299,970 \\
290,922 \\
299,346 \\
306,641 \\
307,313\end{array}$ & $\mid$ & $\begin{array}{l}359,700 \\
348,600 \\
358,200 \\
367,100 \\
371,600\end{array}$ \\
\hline $\begin{array}{l}1931 \\
1932 \\
1933 \\
1934 \\
1935\end{array}$ & $\begin{array}{l}313,654 \\
322,974 \\
320,352 \\
327,309 \\
333,190\end{array}$ & - & $\begin{array}{l}376,600 \\
386,500 \\
384,800 \\
392,100 \\
398,400\end{array}$ \\
\hline $\begin{array}{l}1936 \\
1937 \\
1938 \\
1939 \\
1940\end{array}$ & $\begin{array}{l}325,819 \\
340,489 \\
336,405 \\
336,121 \\
335,075\end{array}$ & $\begin{array}{r}60,920,000 \\
59,580,000\end{array}$ & $\begin{array}{l}389,500 \\
408,400 \\
406,800 \\
404,800 \\
402,100\end{array}$ \\
\hline $\begin{array}{l}1941 \\
1942 \\
1943 \\
1944 \\
1945 \\
1946\end{array}$ & $\begin{array}{l}333,581 \\
329,133 \\
335,935 \\
338,473 \\
341,354 \\
346,700\end{array}$ & $\begin{array}{l}52,590,000 \\
69,180,000 \\
70,420,000 \\
46,190,000 \\
56,600,000 \\
74,760,000\end{array}$ & $\begin{array}{l}400,400 \\
397,800 \\
403,800 \\
407,400 \\
410,800 \\
417,500\end{array}$ \\
\hline
\end{tabular}

COLUMBIA RIVER, INTERNATIONAL BOUNDARY TO GRAND COULEE DAM

\section{KETTLE RIVER}

There is little irrigation in the United States portion of the Kettle River basin. At present, about 880 acres are under irrigation, mostly in the upper reaches above Laurier, Wash. The 1940 Canada Yearbook lists 2,090 acres under irrigation in the Canadian portion of the basin. The following table shows the irrigated area in the Kettle River basin in the United States, as determined primarily from census figures. The net consumptive use for this subdivision was estimated to be 1.25 acre-feet per acre. 
Irrigated area, in acres, in Kettle River basin in the United States

\begin{tabular}{l|c|r}
\hline Year & $\begin{array}{c}\text { Kettle River near } \\
\text { Laurier, Wash. }\end{array}$ & $\begin{array}{c}\text { Kettle River } \\
\text { at mouth }\end{array}$ \\
\hline 1890 & 100 & $\mathbf{1 2 5}$ \\
1900 & 280 & 345 \\
1905 & 230 & 280 \\
1910 & 210 & 250 \\
1915 & 300 & 365 \\
1920 & 390 & 475 \\
1921 & & \\
1922 & 415 & 505 \\
1923 & 435 & 530 \\
1924 & 460 & 560 \\
1925 & 480 & 585 \\
1926 & 505 & 615 \\
1927 & & \\
1928 & 525 & 640 \\
1929 & 550 & 670 \\
1930 & 570 & 695 \\
1931 & 590 & 720 \\
1932 & 600 & 735 \\
1933 & & 750 \\
1934 & 615 & 765 \\
1935 & 625 & 780 \\
1936 & 640 & 795 \\
1937 & 650 & 810 \\
1938 & 665 & 825 \\
1939 & & 840 \\
1940 & 675 & 860 \\
1941 & 690 & 880 \\
1942 & 705 & 880 \\
1943 & 720 & 880 \\
1944 & 720 & 880 \\
1946 & 720 & 880 \\
\hline & 720 & 880 \\
& 720 & 880 \\
& 720 & 880 \\
\hline
\end{tabular}

\section{COLVILLE RIVER}

Little irrigating has been done in the Colville River basin, and only a small percentage of all the land within the basin is classed as irrigable. The water requirements for this area are largely satisifed under natural conditions, such as precipitation and flooding without adequate drainage. At present all irrigation is done by individuals, but at one time two irrigation districts diverted water from Colville River below the hydroelectric plant at Kettle Falls to irrigate lands along the Columbia River.

The Fruitland Irrigation Co. started about 1908 with 1,200 acres (downstream from Kettle Falls), but the acreage diminished to 500 in 1925. This latter acreage was irrigated until about 1937 when the district was dissolved because it was within the reservoir area of Grand Coulee Dam. The Columbia Irrigation District (Plantation Irrigation Co.), during 1910 and 1911 irrigated a.bout 1, 000 acres of land just upstream from Colville River. 
Return flow from presently irrigated land is believed to reenter the Colville River above the gaging station at Kettle Falls, although from 1908 to 1937 the return flow from Fruitland Irrigation Co. was direct to the Columbia River downstream from the mouth of the Colville River. The irrigated area shown in the following table was determined from water rights, census reports, ard local inquiry. The net consumptive use for this tributary basin was estimated to be 1.5 acre-feet per acre.

\section{SPOKANE RIVER}

Irrigation in the Spokane River basin started during the latter part of the nineteenth century but developed slowly until about 1905. From 1905-15 expansion was rapid, primarily just east of Spokane. The region was organized into small irrigation districts and companies, a total of 30 having been active at various times. The three largest were the Spokane Valley, Vera, ant Otis Orchards irrigation districts. These districts serve approximately $4,360,2,300$, and 2, 700 acres respectively. About one-third of the districts derived their water from wells; the remainder use water from lakes and from the Spokane River, mostly by pumping. The irrigated area for this basin was computed primarily from reports of the Idaho State Engineer and records of the Spokane County Treasurer, supplemented by information obtained from officials of the privately operated irrigation districts and companies, and from Fred J. Cunningham, Spokane attorney. The course and seasonal distribution of the return flow from the irrigated acreages in this basin is not well defined owing to the presence of extensive beds of highly permeable material. The Geological Survey has been investigating ground-water movement through portions of this basin and a preliminary report was issued as Water-Supply Paper 889-B. Because return-flow conditions are unknown, no attempt has been made in this report to estimate depletion at any of the gaging stations in this basin. However, for purposes of computing depletion at sites downstream on the Columbia River, a net consumptive use of 1.35 acre-feet per acre was used. This was estimated on the basis of computations by Lowry-Johnson method. Irrigated area above selected locations in the Spokane River basin are shown in the following table. 
Irrigated area, in acres, for indicated year

\begin{tabular}{|c|c|c|c|c|}
\hline Year & $\begin{array}{c}\text { Colville River } \\
\text { basin }\end{array}$ & $\begin{array}{l}\text { Spokane River at } \\
\text { Post Falls, Idaho }\end{array}$ & $\begin{array}{c}\text { Spokane River } \\
\text { at Spokane, } \\
\text { Wash. }\end{array}$ & $\begin{array}{l}\text { Spokane River } \\
\text { at mouth }\end{array}$ \\
\hline $\begin{array}{l}1890 \\
1900\end{array}$ & $\begin{array}{r}360 \\
1,400\end{array}$ & $\mid \ldots \ldots \ldots \ldots \ldots \ldots \ldots \ldots \ldots \ldots \ldots \ldots \ldots \ldots \ldots$ & 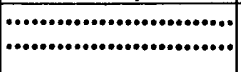 & 500 \\
\hline $\begin{array}{l}1905 \\
1910 \\
1915 \\
1920\end{array}$ & $\begin{array}{l}1,900 \\
5,400 \\
4,560 \\
4,630\end{array}$ & $\begin{array}{ll}272 \\
& 272 \\
& 272\end{array}$ & $\begin{array}{r}1,120 \\
10,864 \\
15,824 \\
18,474\end{array}$ & $\begin{array}{r}2,005 \\
13,120 \\
22,840 \\
26,240\end{array}$ \\
\hline $\begin{array}{l}1921 \\
1922 \\
1923 \\
1924 \\
1925\end{array}$ & $\begin{array}{l}4,270 \\
3,910 \\
3,550 \\
3,180 \\
2,820\end{array}$ & $\begin{array}{l}272 \\
212 \\
212 \\
212 \\
212\end{array}$ & $\begin{array}{l}18,594 \\
18,874 \\
24,255 \\
24,294 \\
23,026\end{array}$ & $\begin{array}{l}26,470 \\
26,850 \\
31,800 \\
28,680 \\
25,250\end{array}$ \\
\hline $\begin{array}{l}1926 \\
1927 \\
1928 \\
1929 \\
1930\end{array}$ & $\begin{array}{l}2,760 \\
2,700 \\
2,640 \\
2,580 \\
2,740\end{array}$ & $\begin{array}{l}212 \\
212 \\
212 \\
212 \\
212\end{array}$ & $\begin{array}{l}23,169 \\
23,119 \\
22,749 \\
22,421 \\
22,369\end{array}$ & $\begin{array}{l}24,990 \\
24,930 \\
24,560 \\
24,230 \\
24,190\end{array}$ \\
\hline $\begin{array}{l}1931 \\
1932 \\
1933 \\
1934 \\
1935\end{array}$ & $\begin{array}{l}2,890 \\
3,040 \\
3,200 \\
3,360 \\
3,510\end{array}$ & $\begin{array}{l}212 \\
212 \\
212 \\
212 \\
212\end{array}$ & $\begin{array}{l}22,539 \\
22,679 \\
22,796 \\
22,373 \\
22,659\end{array}$ & $\begin{array}{l}24,370 \\
24,520 \\
24,650 \\
24,230 \\
24,530\end{array}$ \\
\hline $\begin{array}{l}1936 \\
1937 \\
1938 \\
1939 \\
1940\end{array}$ & $\begin{array}{l}3,660 \\
3,820 \\
3,480 \\
3,630 \\
3,630\end{array}$ & $\begin{array}{l}212 \\
212 \\
212 \\
212 \\
212\end{array}$ & $\begin{array}{l}22,532 \\
22,566 \\
22,586 \\
22,713 \\
22,303\end{array}$ & $\begin{array}{l}24,410 \\
24,460 \\
24,490 \\
24,620 \\
24,210\end{array}$ \\
\hline $\begin{array}{l}1941 \\
1942 \\
1943 \\
1944 \\
1945 \\
1946\end{array}$ & $\begin{array}{l}3,630 \\
3,630 \\
3,690 \\
3,980 \\
4,080 \\
4,080\end{array}$ & $\begin{array}{r}212 \\
212 \\
212 \\
212 \\
212 \\
1,492\end{array}$ & $\begin{array}{l}22,316 \\
22,308 \\
22,110 \\
21,347 \\
21,339 \\
22,646\end{array}$ & $\begin{array}{l}24,230 \\
24,220 \\
24,020 \\
23,260 \\
23,250 \\
24,560\end{array}$ \\
\hline
\end{tabular}

\section{COLUMBIA RIVER AT GRAND COULEE DAM, WASH.}

Outside the major subdivisions already described, a small amount of land is irrigated with water diverted from minor tributaries of the Columbia River and water pumped directly from the river. Very little specific information is available regarding the se tracts, and most of the data have been derived from census reports supplemented by information from residents and from the files of the Nespelem Indian Agency. This agency ororates two small irrigation projects on Hall and Stranger Creeks. The return flow from the lands irrigated in this portion of the basin enters the Columbia River main stem above Grand Coulee. The net consumptive use for lands in this portion of the basir was estimated to be 1.35 acre-feet per acre. The percentage c'epletion of the yield of the basin above Grand Coulee Dam for the Feriod 1921 through 1945 was estimated to be 0.6 percent. The following table shows irrigated area, annual runoff, and estimated depletion for the basin above Grand Coulee Dam, Wash. Pumping from Franklin D. Roosevelt Lake for irrigation of lands in the Columbia Basin project is expected to begin in 1951 . 
Irrigated area, annual runoff, and estimated depletion for Columbia River at Grand Coulee

\begin{tabular}{|c|c|c|c|}
\hline Year & $\begin{array}{l}\text { Irrigated area } \\
\text { (acres) }\end{array}$ & $\begin{array}{l}\text { Runoff } \\
\text { (acre-feet) }\end{array}$ & $\begin{array}{c}\text { Estimated } \\
\text { depletion } \\
\text { (acre-feet) }\end{array}$ \\
\hline $\begin{array}{l}1860 \\
1870 \\
1880 \\
1890 \\
1900\end{array}$ & $\begin{array}{r}1,000 \\
10,000 \\
26,000 \\
75,210 \\
191,813\end{array}$ & : & $\begin{array}{r}1,400 \\
11,500 \\
28,400 \\
78,500 \\
226,100\end{array}$ \\
\hline $\begin{array}{l}1905 \\
1910\end{array}$ & $\begin{array}{l}254,526 \\
288,442\end{array}$ & 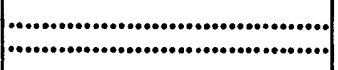 & $\begin{array}{l}305,600 \\
354,200\end{array}$ \\
\hline $\begin{array}{l}1914 \\
1915\end{array}$ & 310,682 & $\begin{array}{l}81,400,000 \\
70,100,000\end{array}$ & $\cdots \ldots \ldots \ldots \ldots$ \\
\hline $\begin{array}{l}1916 \\
1917 \\
1918 \\
1919\end{array}$ & 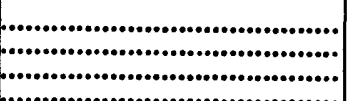 & $\begin{array}{l}98,000,000 \\
82,800,000 \\
85,700,000\end{array}$ & ( \\
\hline 1920 & 336,354 & $71,800,000$ & $\cdots \cdots \cdots \cdots$ \\
\hline $\begin{array}{l}1921 \\
1922 \\
1923 \\
1924 \\
1925\end{array}$ & $\begin{array}{l}333,244 \\
344,615 \\
334,775 \\
339,056 \\
332,732\end{array}$ & $\begin{array}{l}91,700,000 \\
74,900,000 \\
79,000,000 \\
62,600,000 \\
91,000,000\end{array}$ & $\begin{array}{l}401,300 \\
420,200 \\
408,600 \\
409,300 \\
405,400\end{array}$ \\
\hline $\begin{array}{l}1926 \\
1927 \\
1928 \\
1929 \\
1930\end{array}$ & $\begin{array}{l}331,570 \\
322,122 \\
329,646 \\
335,781 \\
336,723\end{array}$ & $\begin{array}{r}53,200,000 \\
91,400,000 \\
103,000,000 \\
58,700,000 \\
61,400,000\end{array}$ & $\begin{array}{l}402,700 \\
391,100 \\
399,400 \\
406,800 \\
411,600\end{array}$ \\
\hline $\begin{array}{l}1931 \\
1932 \\
1933 \\
1934 \\
1935\end{array}$ & $\begin{array}{l}343,544 \\
353,354 \\
351,132 \\
357,979 \\
364,460\end{array}$ & $\begin{array}{r}58,500,000 \\
82,800,000 \\
91,400,000 \\
101,400,000 \\
81,440,000\end{array}$ & $\begin{array}{l}417,300 \\
427,900 \\
426,400 \\
433,900 \\
441,100\end{array}$ \\
\hline $\begin{array}{l}1936 \\
1937 \\
1938 \\
1939 \\
1940\end{array}$ & $\begin{array}{l}357,269 \\
372,299 \\
368,555 \\
368,701 \\
367,245\end{array}$ & $\begin{array}{l}70,830,000 \\
57,500,000 \\
81,960,000 \\
69,280,000 \\
\$ 67,800,000\end{array}$ & $\begin{array}{l}432,400 \\
451,800 \\
450,600 \\
449,200 \\
446,000\end{array}$ \\
\hline $\begin{array}{l}1941 \\
1942 \\
1943 \\
1944 \\
1945 \\
1946\end{array}$ & $\begin{array}{l}365,771 \\
361,313 \\
367,975 \\
370,043 \\
373,014 \\
379,670\end{array}$ & 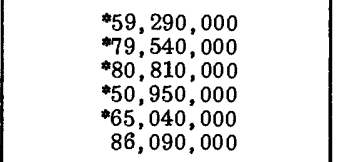 & $\begin{array}{l}443,800 \\
441,700 \\
447,500 \\
450,500 \\
454,100 \\
462,500\end{array}$ \\
\hline
\end{tabular}

* Adjusted for change in contents in Franklin D. Roosevelt Lake.

\section{COLUMBIA RIVER, GRAND COULEE DAM TO SNAKE RIVER}

\section{OKANOGAN RIVER}

The land currently irrigated in the United States portion of the Okanogan River basin totals about 22, 000 acres, a large part lying in narrow, discontinuous strips along the main river channel.

Three irrigation districts provide water by gravity to approximately half the irrigated area. The Whitestone Project, near Loomis, Wash., which includes about 2,000 acres, is an offstream development and obtains water from Toats Coulee Creek. 
Blue, Spectacle, and Whitestone Lakes provide storage for this project. Originally it was planned to develop over 10,000 acres of irrigated land but due to insufficient water the project shrank to its present size. The Okanogan Project of the Bureau of Reclamation includes about 3,800 acres of land between Johnson Creek and Salmon Creek. Water is diverted from Salmon Creek, and storage is provided by Conconully Lake. At one time, a.bout 8,000 acres were irrigated, but owing to an inadequate water supply, the area has been reduced to its present size. The largest irrigation project in the basin is the Oroville-Tonasket Irrigation District, which diverts water from the Similkameen River between Nighthawk and Oroville, Wash. The irrigated land lies on both sides of Okanogan River from the international boundary to and just south of Tonasket, Washington, and includes about 7,000 acres. Many small private and company-owned pumping plants supply water to the bench lands along the Okanogan River.

Irrigation in the Similkameen River basin is located around Palmer Lake and the Sinlahekin Valley. The Canada Yearbook for 1940 shows 34,848 acres of irrigated land in the Canadian portion of the Okanogan River basin. Most of this land is irrigated by direct diversion from the Okanogan River. Wa ter stored in Okanagan Lake is used to supplement the late summer streamflows for the irrigated area near Oliver, British Colurnbia.

The following table shows the irrigated area in the United States portion of the Okanogan River basin. These data wer? obtained from irrigation district reports, census reports, records of watermasters, and from pioneer residents. All return flow is believed to enter the Okanogan River above its mouth except that from about 500 acres of the Monse Indian project, which enters the Columbia River directly. The average net consumptive use for all lands in this subdivision was estimated to be 1.5 acre-feet per acre. 
Irrigated area, in acres, for Okanogan River basin in the United Stater

\begin{tabular}{|c|c|c|c|}
\hline Year & $\begin{array}{l}\text { Similkameen River } \\
\text { near Nighthawk, } \\
\text { Wash. }\end{array}$ & $\begin{array}{l}\text { Okanogan River } \\
\text { near Tonasket, } \\
\text { Wash. }\end{array}$ & $\begin{array}{l}\text { Okanogan River } \\
\text { at mouth }\end{array}$ \\
\hline $\begin{array}{l}1860 \\
1870 \\
1880 \\
1890 \\
1900\end{array}$ & $\begin{array}{r}205 \\
470 \\
1,480\end{array}$ & $\begin{array}{r}20 \\
20 \\
225 \\
490 \\
1,560\end{array}$ & $\begin{array}{r}20 \\
20 \\
225 \\
960 \\
2,100\end{array}$ \\
\hline $\begin{array}{l}1905 \\
1910 \\
1915 \\
1920\end{array}$ & $\begin{array}{l}1,500 \\
1,580 \\
1,580 \\
2,580\end{array}$ & $\begin{array}{r}1,670 \\
1,760 \\
2,760 \\
10,800\end{array}$ & $\begin{array}{r}2,230 \\
7,940 \\
12,500 \\
18,850\end{array}$ \\
\hline $\begin{array}{l}1921 \\
1922 \\
1923 \\
1924 \\
1925\end{array}$ & $\begin{array}{l}2,780 \\
2,880 \\
2,980 \\
3,020 \\
3,100\end{array}$ & $\begin{array}{l}11,000 \\
11,100 \\
11,200 \\
11,200 \\
11,100\end{array}$ & $\begin{array}{l}19,300 \\
19,300 \\
19,100 \\
19,700 \\
19,800\end{array}$ \\
\hline $\begin{array}{l}1926 \\
1927 \\
1928 \\
1929 \\
1930\end{array}$ & $\begin{array}{l}3,100 \\
3,060 \\
2,980 \\
2,940 \\
2,940\end{array}$ & $\begin{array}{l}11,100 \\
11,000 \\
10,900 \\
11,000 \\
10,900\end{array}$ & $\begin{array}{l}20,000 \\
19,400 \\
19,400 \\
19,800 \\
19,700\end{array}$ \\
\hline $\begin{array}{l}1931 \\
1932 \\
1933 \\
1934 \\
1935\end{array}$ & $\begin{array}{l}2,940 \\
3,000 \\
3,000 \\
3,040 \\
3,040\end{array}$ & $\begin{array}{l}10,800 \\
10,800 \\
10,700 \\
10,700 \\
10,700\end{array}$ & $\begin{array}{l}19,600 \\
19,300 \\
19,300 \\
19,300 \\
19,400\end{array}$ \\
\hline $\begin{array}{l}1936 \\
1937 \\
1938 \\
1939 \\
1940\end{array}$ & $\begin{array}{l}3,040 \\
3,200 \\
3,160 \\
3,120 \\
3,180\end{array}$ & $\begin{array}{l}10,700 \\
10,900 \\
10,800 \\
10,700 \\
10,700\end{array}$ & $\begin{array}{l}19,200 \\
19,300 \\
19,600 \\
19,100 \\
19,300\end{array}$ \\
\hline $\begin{array}{l}1941 \\
1942 \\
1943 \\
1944 \\
1945 \\
1946\end{array}$ & $\begin{array}{l}3,050 \\
3,010 \\
2,970 \\
2,970 \\
2,930 \\
2,930\end{array}$ & $\begin{array}{l}10,600 \\
10,600 \\
10,600 \\
10,600 \\
10,700 \\
10,700\end{array}$ & $\begin{array}{l}19,100 \\
19,400 \\
19,800 \\
20,200 \\
20,500 \\
22,100\end{array}$ \\
\hline
\end{tabular}

\section{METHOW RIVER}

The earlier inhabitants of the Methow Valley were primerily interested in mining and in raising livestock. Some ditches were first constructed in connection with mining, and after the turn of the century they were converted to agricultural purposes. Most of the irrigated lands lie along the river in small tracts, and water is supplied by small ditche maintained under individual or partner ownership. The majority of the expansion took place between 1905 and 1910 .

The China Ditch, near Pateros, was the first known diversion in the Methow Valley. It was originally used for placer mining, but irrigation probably started about 1900. There was little expansion in this area until a district was organized in 1922. Since that time water has been supplied to about 330 acres, along the 
Columbia River downstream from Methow River. The Pateros Ditch was built in 1903, and in 1904 delivered water to 300 acres, which later was reduced to about 250 acres. The district was formed in 1916 to include land lying along the north side of Methow River and the Columbia River upstream from the confluence of these streams.

The Methow Canal Co. diverted water from Twisp and Methow Rivers to irrigate land along the lower 4 miles of Trisp River and the 10 miles immediately downstream from the confluence of that stream with the Methow River. The first irrigation water was delivered in 1909. In 1919 the Methow Canal Co. became the Methow Valley Irrigation District, which presently irrigates about 1,900 acres.

The Wolf Creek Irrigation District was formed in 1922 to irrigate landin the vicinity of Winthrop. Water is diverted from Wolf Creek and stored in Patterson Lake for about 650 acres.

The discharge records of the gaging station on Methow River at Twisp do not represent the yield of the basin at that point. Two canals of the Methow Valley Irrigation District and Risley ditch divert water around the station. The gaging station on Methow River at Pateros, is essentially at the mouth of the stream, and except as has been indicated all return flow is believed to enter the stream above this point.

The irrigated area in this basin, shown in the following table, is based primarily on records of existing irrigation districts, adjudicated water rights, and census reports. The distribution of private irrigated lands above and below Twisp was kased on a survey made by the Washington State Department of Conservation and Development in 1924. Field investigations by the Geological Survey in 1915 and 1947 were also used. The net consumptive use for this basin was estimated to be 1.75 acre-feet per acre. 
Irrigated area, annual runoff, and estimated depletion for Methow River basin

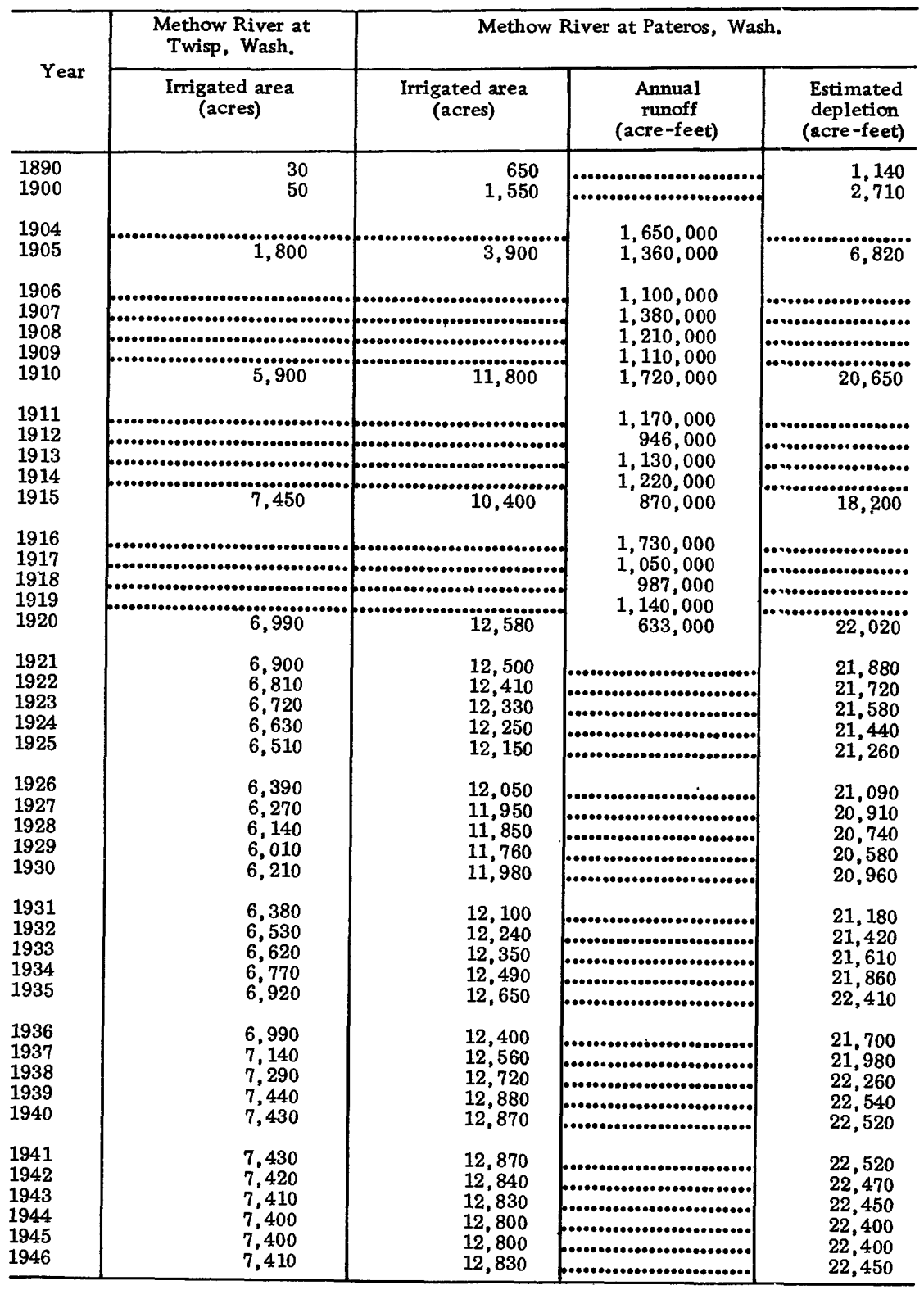




\section{CHELAN RIVER}

Owing to the rugged terrain surrounding Lake Chelan and to the use of the lake to store water for generating hydroelectric power the amount of irrigation within the Chelan River basin is relatively small. Most of the irrigated lands are in organized districts. The earliest irrigation was on the south side of Lake Chelan near Manson; water was diverted from First Creek, and about 100 acres have been under irrigation since 1907. This area was organized in 1922 as the First Creek Irrigation District.

The Chelan Falls Irrigation District started the following year on 300 acres on the west bank of the Columbia River with water diverted from Lake Chelan.

The Lake Chelan Reclamation District, on the north side of Lake Chelan near Manson, constitute the largest block of irrigated land in the basin. The districtwas started with about 400 acres in 1909. The increase was gradual up to about 2, 000 acres in 1926 , then 1,000 acres were added in 1927 to make a total of 3,000 acres. At the present time about 3,600 acres are irrigated from the waters of seven mountain streams, storage being provided by several small lakes.

The Isenhart Irrigation District was started in 1931 and irrigates 250 acres just northeast of Lake Chelan. Water is pumped from the lake.

The irrigated acreages shown in the following table have been estimated on the basis of records of the irrigation districts and by field investigations, which included the collection of information from pioneers of the area. Officials of the Washington Water Power Co. supplied information concerning the amount of land served since the development of their hydroelectric plant at Chelan Falls. The netconsumptive use for this basin was estimated to be 1. 75 acre-feet per year.

279538 o $-53-4$ 
Irrigated area, and estimated depletion for Chelan River basin, including land irrigated with Chelan River water

\begin{tabular}{l|r|r}
\hline Year & $\begin{array}{c}\text { Irrigated area } \\
\text { (acres) }\end{array}$ & $\begin{array}{r}\text { Estimated } \\
\text { depletion } \\
\text { (acre-feet) }\end{array}$ \\
\hline 1910 & 1,610 & $\mathbf{2}, 820$ \\
1915 & 2,500 & 4,380 \\
1920 & 3,360 & 5,880 \\
1921 & 3,500 & 6,120 \\
1922 & 3,650 & 6,390 \\
1923 & 3,790 & 6,630 \\
1924 & 3,930 & 6,880 \\
1925 & 4,080 & 7,140 \\
1926 & 4,220 & 7,380 \\
1927 & 5,260 & 9,200 \\
1928 & 5,330 & 9,330 \\
1929 & 5,380 & 9,420 \\
1930 & 5,430 & 9,500 \\
1931 & 5,730 & 10,030 \\
1932 & 5,760 & 10,080 \\
1933 & 5,800 & 10,150 \\
1934 & 5,840 & 10,220 \\
1935 & 5,870 & 10,270 \\
1936 & 5,920 & 10,360 \\
1937 & 5,960 & 10,430 \\
1938 & 6,020 & 10,540 \\
1939 & 6,060 & 10,600 \\
1940 & 6,110 & 10,690 \\
1941 & 6,140 & 10,740 \\
1942 & 6,160 & 10,780 \\
1943 & 6,190 & 10,830 \\
1944 & 6,220 & 10,880 \\
1945 & 6,280 & 10,940 \\
1946 & & 10,990 \\
\hline
\end{tabular}

\section{ENTIAT RIVER}

Owing to its mountainous nature there has been relatively little irrigation development in the Entiat River basin. Most of the land is irrigated from privately owned ditches and lies along the lower reaches of the river above the gaging station at Entiat, which is essentially at the mouth of the river. The exception is the Entiat Irrigation District, formed in 1920, where water is diverted from the river above the gaging station at Entiat to irrigate about 600 acres lying below the gaging station along the west kank of the Columbia River. Past records of this diversion are not available, and the canal flow is not included in the gaging-staticn record.

The following table shows data for the Entiat River. The return flow from all lands except the Entiat Irrigation District is assumed to reach the river above its confluence with the Columbia River. The net consumptive use for this basin was estimated to be 1.75 acre-feet per acre. 
Irrigated area, annual runoff, and estimated depletion, for Entiat River at Entiat, Wash.

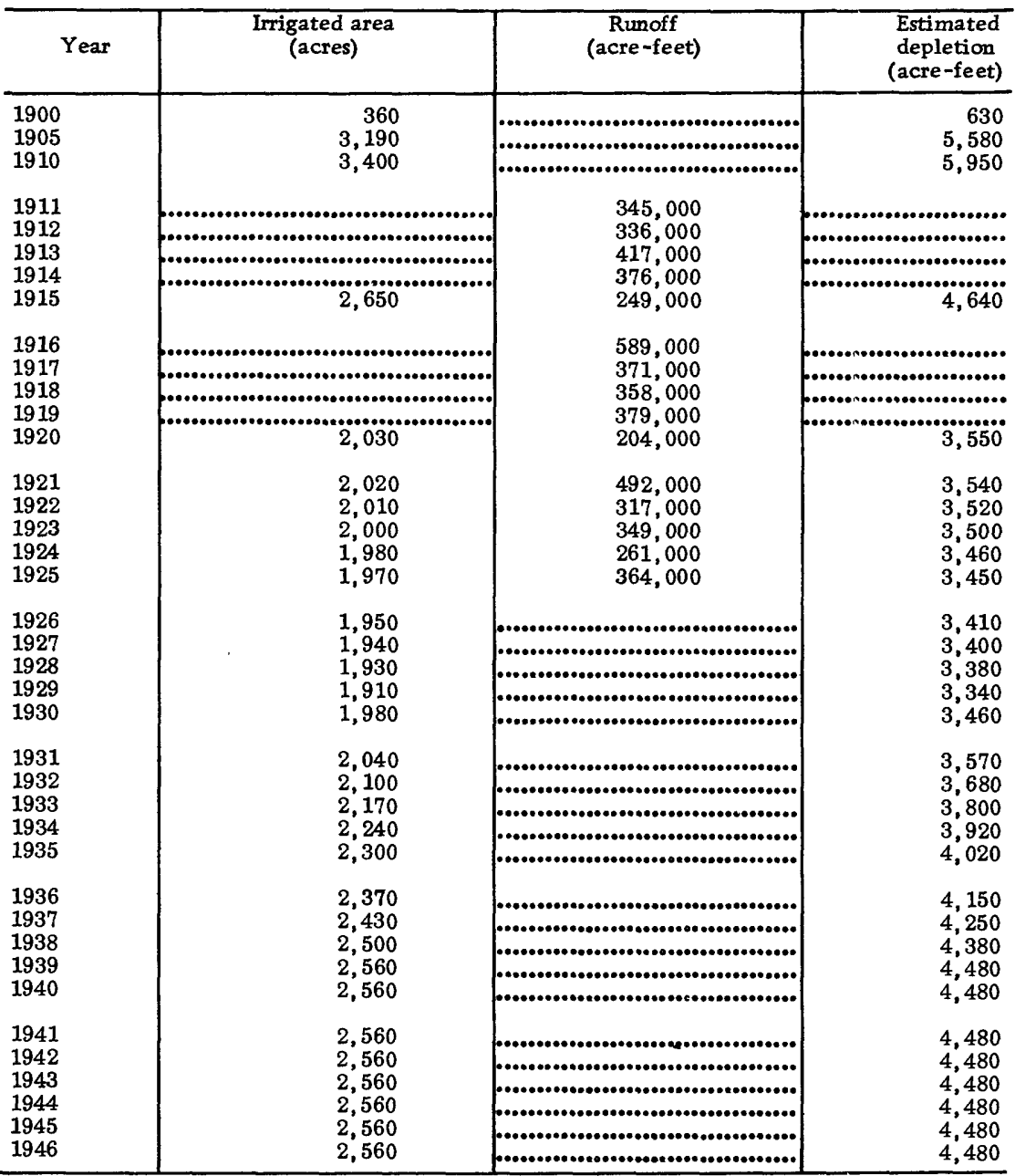

\section{WENATCHEE RIVER}

Irrigation has been practiced in the Wenatchee River valley of Washington from the time of the earliest white settlers, beginning on a small scale with the construction of Miller Ditch about 1870 , to divert water from Squillchuck Creek. During the next few years several other small ditches were constructed on tributary streams, but no large ditches were built until 1891, when work was started on the Gunn Ditch, which diverts water from Wenatchee River about 1 mile above Monitor. By 1898, this ditch had beon extended as far as Wenatchee and served about 1,000 acres including some lands along the Columbia River above and below the mouth of the Wenatchee River. 
The Peshastin Ditch was built about 1898 to irrigate about 1,900 acres of land near the mouth of the Peshastin Creek and on the south side of Wenatchee River between Dryden and Cashmere. The Peshastin Irrigation District took over the operation of this canal in 1917, and now serves about 3,600 acres.

The largest project in the basin is the Wenatchee Feclamation District which was formed in 1915. Hiline Canal, tro principal canal of this district, was constructed during the period 1902 to 1907. Water is diverted above Dryden, and carried down the north bank of the river to a point near the mouth of the Wenatchee River; there the canal divides, one branch extending a short distance up the west bank of the Columbia and the other extending cownstream along the Columbia into Douglas County. About 11,500 acres are served by this canal.

The Jones-Shotwell ditch, completed in 1903, has its intake 1 mile above Cashmere, and serves about 700 acres alc $n g$ the east side of Wenatchee River. The Icicle Irrigation District was formed in 1917, although water had been delivered by the Icicle Canal Co. since 1910. With about 1,000 acres in the beginning, tre company now serves about 3,000 acres on both sides of Wenatchee River near Leavenworth, and downstream as far as Mission Creek.

There has been comparatively little new development in irrigation since about 1915 except for the Wenatchee-Chiwawa Irrigation District, which was formed in 1923, and now irrigates about 1,400 acres near Plain.

The following table shows the data on Wenatchee River basin. The irrigated areas were determined from reports of irrigation districts, supplemented by information from local picneer residents, county tax records, and census reports. The return flow from the irrigated lands in this subdivision is partly direct to the Wenatchee River and part directly to the Columbia, above the dam at Rock Island. The net consumptive use as computed by LowryJohnson method is 2.0 acre-feet per acre. 
Irrigated area and estimated depletion for Wenatchee River basin

\begin{tabular}{|c|c|c|c|}
\hline \multirow{2}{*}{ Year } & \multirow{2}{*}{$\begin{array}{c}\begin{array}{c}\text { Wenatchee River } \\
\text { at Peshastin, Wash. }\end{array} \\
\begin{array}{c}\text { Irrigated area } \\
\text { (acres) }\end{array}\end{array}$} & \multicolumn{2}{|c|}{$\begin{array}{l}\text { Wenatchee River } \\
\text { at mouth }\end{array}$} \\
\hline & & $\begin{array}{l}\text { Irrigated area } \\
\text { (acres) }\end{array}$ & $\begin{array}{l}\text { Estimated } \\
\text { depletion } \\
\text { (acre-feet) }\end{array}$ \\
\hline 1900 & [................................................... & 3,100 & 6,200 \\
\hline $\begin{array}{l}1905 \\
1910 \\
1915 \\
1920\end{array}$ & $\begin{array}{r}650 \\
760 \\
1,430 \\
1,740\end{array}$ & $\begin{array}{l}12,850 \\
19,210 \\
23,470 \\
23,180\end{array}$ & $\begin{array}{l}25,700 \\
38,420 \\
46,940 \\
46,360\end{array}$ \\
\hline $\begin{array}{l}1921 \\
1922 \\
1923 \\
1924 \\
1925\end{array}$ & $\begin{array}{l}1,770 \\
1,820 \\
3,160 \\
3,190 \\
3,230\end{array}$ & $\begin{array}{l}23,150 \\
23,180 \\
24,520 \\
24,530 \\
24,550\end{array}$ & $\begin{array}{l}46,300 \\
46,360 \\
49,030 \\
49,060 \\
49,100\end{array}$ \\
\hline $\begin{array}{l}1926 \\
1927 \\
1928 \\
1929 \\
1930\end{array}$ & $\begin{array}{l}3,280 \\
3,320 \\
3,360 \\
3,350 \\
3,350\end{array}$ & $\begin{array}{l}24,560 \\
24,590 \\
24,630 \\
24,600 \\
24,650\end{array}$ & $\begin{array}{l}49,120 \\
49,180 \\
49,260 \\
49,200 \\
49,300\end{array}$ \\
\hline $\begin{array}{l}1931 \\
1932 \\
1933 \\
1934 \\
1935\end{array}$ & $\begin{array}{l}3,310 \\
3,260 \\
3,220 \\
3,170 \\
3,130\end{array}$ & $\begin{array}{l}24,700 \\
24,700 \\
24,740 \\
24,760 \\
24,790\end{array}$ & $\begin{array}{l}49,400 \\
49,400 \\
49,480 \\
49,520 \\
49,580\end{array}$ \\
\hline $\begin{array}{l}1936 \\
1937 \\
1938 \\
1939 \\
1940\end{array}$ & $\begin{array}{l}3,060 \\
3,120 \\
3,120 \\
3,180 \\
3,200\end{array}$ & $\begin{array}{l}24,780 \\
24,910 \\
24,980 \\
25,020 \\
25,190\end{array}$ & $\begin{array}{l}49,560 \\
49,820 \\
49,960 \\
50,040 \\
50,380\end{array}$ \\
\hline $\begin{array}{l}1941 \\
1942 \\
1943 \\
1944 \\
1945 \\
1946\end{array}$ & $\begin{array}{l}3,230 \\
3,250 \\
3,290 \\
3,280 \\
3,260 \\
3,240\end{array}$ & $\begin{array}{l}25,300 \\
25,370 \\
25,490 \\
25,500 \\
25,490 \\
25,470\end{array}$ & $\begin{array}{l}50,600 \\
50,740 \\
50,980 \\
51,000 \\
50,980 \\
50,940\end{array}$ \\
\hline
\end{tabular}

COLUMBIA RIVER AT TRINIDAD, WASH.

In addition to the major tributary basins between Grand Coulee Dam and the gaging station on Columbia River at Trinidad, already discussed, there is additional irrigation along the main stem of the Columbia River, utilizing water from minor tributaries or pumping directly from the Columbia River. These tracts vary in size from a few hundred acres to about 1,500 acres and are fairly well distributed along the narrow river valley below the Okanogan River. The return flow from all of these lands enters the Columbia River above the gaging station at Trinidad. The net consumptive use for this portion of the basin was estimated to bo 2.0 acrefeet per acre, the same as for the Wenatchee River basin.

The following table shows data for the United States portion of the Columbia River basin above the gaging station at Trinidad, Wash. The percentage depletion, based upon the pariod 1921 through 1945 was computed to be 0.7 percent and has shown only a slight increase during this period. 
Irrigated area, annual runoff, and estimated depletion for Columbia River at Trinidad, Wash.

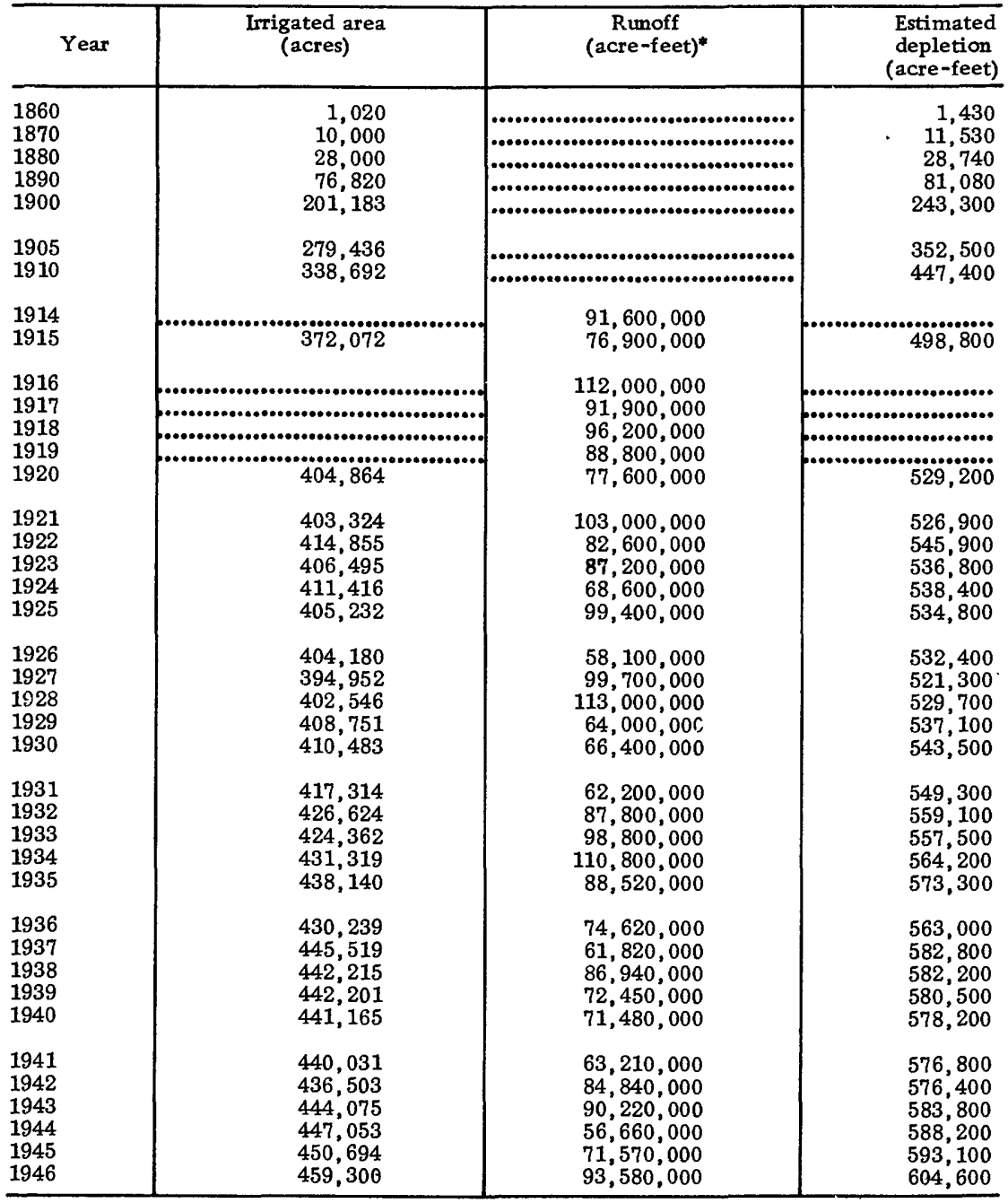

* Adjusted for storage in Franklin D. Roosevelt Lake since October 1939.

\section{CRAB CREEK}

Crab Creek drains a semiaridarea having very low runoff. The stream is discontinuous or intermittent except in certain reaches. The lower portion of the basin encompasses much of the area included in the Columbia Basin Project to be irrigated by pumping from Franklin D. Roosevelt Lake. At present extensive areas in the basinare cultivated by dry-farming methods and profuce grain crops. 
Irrigation in the basin has been limited to small scattered tracts, Moses Lake Irrigation District being the only organized enterprise. This district stores water in Moses Lake and member farmers pump directly from the lake. Other lands are irrigated by water from small tributary streams or springs. The irrigated areas shown in the following table have been compiled from data furnished by pioneers and other local residents, supplemented by census reports.

Movement of ground water in the area is not well known, but it is believed that a considerable amount escapes directly to the Columbia River. The depletion of Crab Creek by irrigation is therefore not estimated in connection with any of the gaging stations within the basin. The net consumptive use for lands in this basin was estimated to be 1.75 acre-feet per acre.

Irrigated area, in acres, at indicated years for Crab Creek basin

\begin{tabular}{|c|c|}
\hline Year & $\begin{array}{l}\text { d area } \\
\text { es) }\end{array}$ \\
\hline $\begin{array}{l}1890 . \\
1900 .\end{array}$ & $\begin{array}{r}330 \\
1,670\end{array}$ \\
\hline $\begin{array}{l}1905 . \\
1910 . \\
1915 . \\
1920 .\end{array}$ & $\begin{array}{l}2,180 \\
3,090 \\
6,110 \\
6,560\end{array}$ \\
\hline $\begin{array}{l}1921 . \\
1922 . \\
1923 . \\
1924 . \\
1925 .\end{array}$ & $\begin{array}{l}6,500 \\
6,440 \\
6,380 \\
6,320 \\
6,260\end{array}$ \\
\hline $\begin{array}{l}1926 . \\
1927 . \\
1928 . \\
1929 . \\
1930 .\end{array}$ & $\begin{array}{l}6,000 \\
5,940 \\
5,880 \\
5,740 \\
5,680\end{array}$ \\
\hline $\begin{array}{l}1931 . \\
1932 . \\
1933 . \\
1934 . \\
1935 .\end{array}$ & $\begin{array}{l}5,620 \\
5,550 \\
5,490 \\
5,430 \\
5,370\end{array}$ \\
\hline $\begin{array}{l}1936 \\
1937 \\
1938 \\
1939 \\
1940\end{array}$ & $\begin{array}{l}5,610 \\
5,850 \\
6,090 \\
6,340 \\
6,640\end{array}$ \\
\hline $\begin{array}{l}1941 . \\
1942 . \\
1943 . \\
1944 . \\
1945 . \\
1946 .\end{array}$ & $\begin{array}{r}6,940 \\
7,240 \\
7,430 \\
9,130 \\
12,130 \\
13,430\end{array}$ \\
\hline
\end{tabular}


YAKMA RIVER

Irrigation agriculture has played a dominant role in the development of the Yakima Valley of central Washington, the largest irrigated area in the Columbia River basin above the Snake River. The earliest irrigation was reputedly started prior to 1860 by Indians, who had been taught the advantage of irrigation by missionaries a few years earlier. There were very few white settlers in the valley before this time. The first authentic report. of irrigation by white men was of the diversion of water from the Naches River through a small ditch constructed by George Nelson in 1867. Through the formation of a small stock company, this ditch was later enlarged to the present Union Canal. The Lauber and Schanno ditch was constructed in 1873 and was extended in 1875 as far as Yakima.

The earliest of the large-scale undertakings was made by the Northern Pacific Railway, which had its western terminus at Yakima for 2 years. In 1890 the company began construstion of the Sunnyside Project to irrigate some 40,000 acres. In 1905 the U. S. Bureau of Reclamation purchased all rights from the Northern Pacific Railway and has extended this district to 105,000 acres, of which about 83,000 acres are currently irrigated. T'is was one of the first projects constructed under the Reclamation Act.

The failure of several private and stock companies during the 1890 's made the financing of storage projects difficult, thus limiting development to land easily accessible to natural river flow. There were 55 principal canals between Cle Elum and the mouth of the Yakima River, including those on the Naches River, plus innumerable small canals on the minor tributaries. This basin has been the focus of intensive development by governmental agencies, until at the present time the projects operated by the Bureau of Reclamation and the Yakima Indian Reservation comprise about 80 percent of all irrigated lands in this basin.

Because of the complex pattern of the irrigation wcrks, no attempt will be made to analyze in detail the projects which have been developed. The Yakima Project, of the Bureau cf Reclamation is comprised of five operating divisions: Sunnyside, Tieton, Kittitas, Roza, and Kennewick divisions. Of these the Sunnyside and Kittitas are the largest. Water is stored in six upstream reservoirs operated by the Bureau of Reclamation to provide irrigation water during low-water seasons. The Wapato Incian Project consists of land which lies mostly on the Indian reservation and which is served by minor tributaries and by the Yakima River. 
The values for irrigated acreage shown in this report have been compiled from the records of the Yakima Project, Wapato Indian Project, and irrigation districts which receive water from these agencies. Records available through the Yakima office of the $\mathrm{Bu}-$ reau of Reclamation were supplemented by data collected by the Geological Survey. These records do not compare clcsely with census figures for the earlier years, probably owing to differences in classification of irrigated land. This was particularly true in the Kittitas Valley where the census figure indicated 30,000 acres irrigated in the earlier years, though Water-Supply Paper 369 indicates that the irrigated lands in this basin received only about one-third of their water requirement. In this report it is assumed that 10,000 acres have been adequately irrigated during the early stage of development. In recent years the irrigated acreage has been based on the records of irrigation districts and irrigation companies, which agree substantially with census figur

The irrigated area above the gaging station on Yakima River near Umtanum, consists mainly of lands in the Kittitas Valley near Ellensburg. These lands receive their water supply principally from the Kittitas Reclamation District and the Cascade Canal Co. supplemented by small district and private irrigation systems. There is a small amount of irrigated land located near Cle Elum adjacent to both the Yakima and Teanaway Rivers. The diversion and return flow from all these lands is assumed to reach the Yakima River above the gaging station near Umtanum.

The irrigated lands above the gaging station on Yakima River at Parker, are those in the main Yakima Valley and the low or portion of the Naches River valley. They are served primarily by works constructed by the Bureau of Reclamation, the Tieton division being the largest single supplier of water in this area. Many small irrigation districts also serve this area. The return flow from all these lands is assumed to reach the Yakima River at or above Union Gap. The discharge measured at the gaging station near Union Gap includes the flow of canals diverting at this point.

The irrigated lands below the gaging station on Yakima River near Parker, and above the gaging station at Kiona corstitute the largest such tract in the Yakima Valley. South. of the river are the lands of the Wapato Indian Project and others to which water is supplied by the Indian Service. The Sunnyside division and the newly created Roza division form the majority of the remaining irrigated area in this portion of the basin. The return flow for all these lands is assumed to reach the Yakima River abore the gaging station at Kiona. 
In addition, a small amount of land is irrigated along the lower reaches of the Yakima River and along the Columbia River near its confluence with the Yakima. These are comprised mainly of the Kennewick division and the Columbia Irrigation District. The return flow from these particular lands is assumed to reach the Columbia River above the mouth of the Snake.

The average net consumptive use for this basin has been estimated to be 2.0 acre-feet per acre and was based upon computations by Lowry-Johnson method, Blaney-Criddle metrod and results of inflow-outflow studies for a portion of the basin. The depletion shown in the following table represents an average depletion of 11. 6 percent of the yield of the watershed above Parker. This is for the period 1921 through 1945. A gradual growth has taken place during this period and in 1946 the depletion represents about 13.6 percent of the long-term yield. Considering the 13year period 1934 through 1946, the percentage depletion above Kiona has averaged 27.2 percent and in 1946 it represents 28. 9 percent of the long-term yield. 


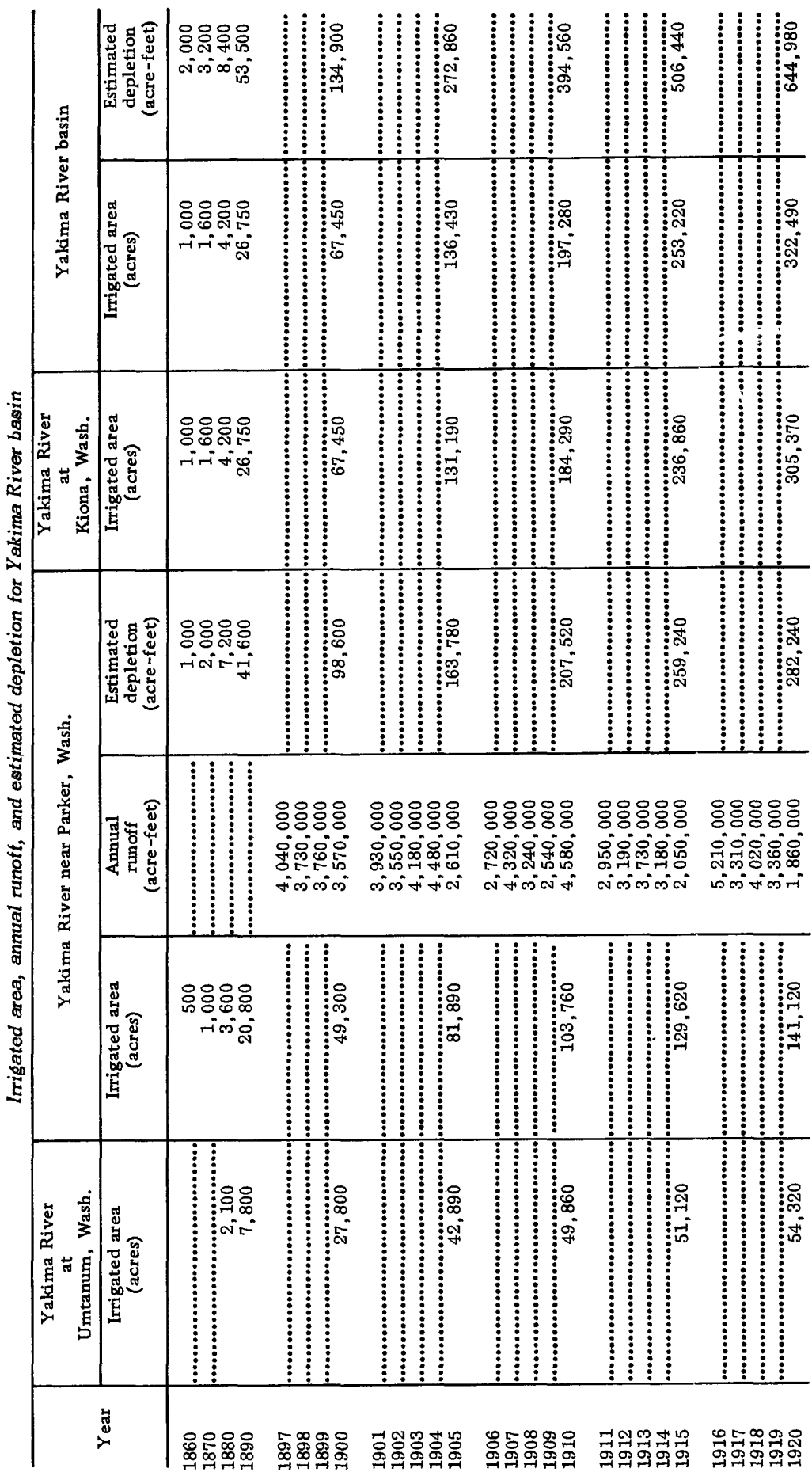




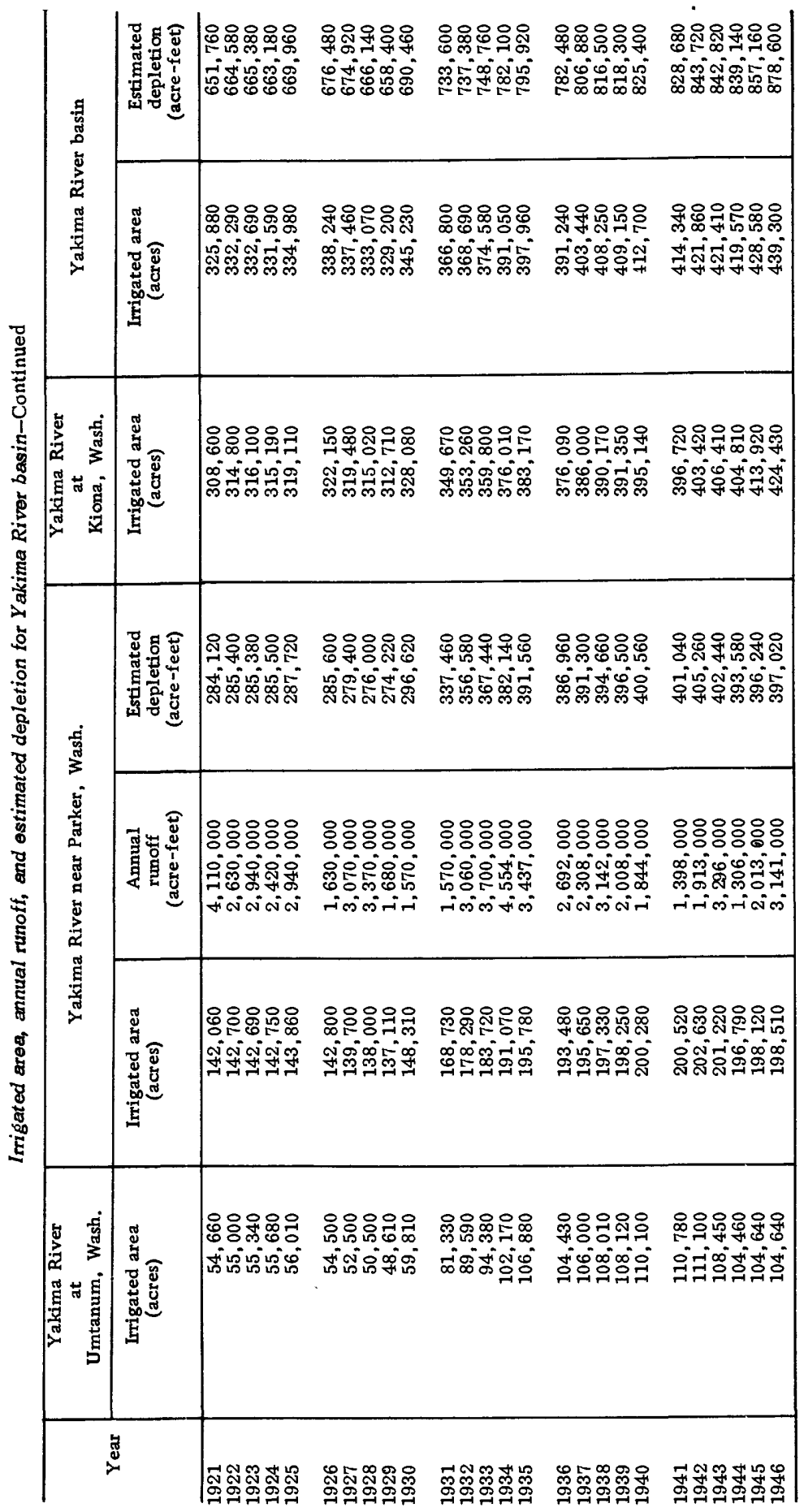




\section{COLUMBIA RIVER, BETWEEN YAKIMA RIVER AND SNAKE RIVEF}

There is a small amount of irrigation along the Columbia River between the Yakima and Snake River, of which the Franl-lin County Irrigation District serves the largest part. The remainder consists of small isolated tracts. The water supply is obtained by pumping from the Columbia River. The return flow from these lands is assumed to reach the Columbia River above the mouth of the Snake.

The irrigated area and estimated depletion for that portion of the Columbia River above the Snake River is summarized in the following table.

Irrigated area and estimated depletion for the Columbia River above mouth of Snake River

\begin{tabular}{|c|c|c|}
\hline Year & $\begin{array}{l}\text { Irrigated area } \\
\text { (acres) }\end{array}$ & $\begin{array}{c}\text { Estimated } \\
\text { depletion } \\
\text { (acre-feet) }\end{array}$ \\
\hline $\begin{array}{l}1860 \\
1870 \\
1880 \\
1890 \\
1900\end{array}$ & $\begin{array}{r}2,020 \\
12,000 \\
32,000 \\
103,900 \\
270,303\end{array}$ & $\begin{array}{r}3,430 \\
14,730 \\
37,140 \\
125,200 \\
381,120\end{array}$ \\
\hline $\begin{array}{l}1905 \\
1910 \\
1915 \\
1920\end{array}$ & $\begin{array}{l}418,636 \\
541,692 \\
634,902 \\
738,134\end{array}$ & $\begin{array}{r}630,300 \\
852,600 \\
1,022,900 \\
1,194,100\end{array}$ \\
\hline $\begin{array}{l}1921 \\
1922 \\
1923 \\
1924 \\
1925\end{array}$ & $\begin{array}{l}739,914 \\
757,795 \\
749,775 \\
754,646 \\
751,592\end{array}$ & $\begin{array}{l}1,198,500 \\
1,230,100 \\
1,221,800 \\
1,223,300 \\
1,225,900\end{array}$ \\
\hline $\begin{array}{l}1926 \\
1927 \\
1928 \\
1929 \\
1930\end{array}$ & $\begin{array}{l}753,740 \\
743,692 \\
746,846 \\
749,051 \\
766,813\end{array}$ & $\begin{array}{l}1,230,000 \\
1,217,300 \\
1,216,800 \\
1,216,300 \\
1,254,700\end{array}$ \\
\hline $\begin{array}{l}1931 \\
1932 \\
1933 \\
1934 \\
1935\end{array}$ & $\begin{array}{l}795,214 \\
806,404 \\
810,102 \\
833,509 \\
847,240\end{array}$ & $\begin{array}{l}1,303,700 \\
1,317,300 \\
1,327,600 \\
1,367,200 \\
1,390,200\end{array}$ \\
\hline $\begin{array}{l}1936 \\
1937 \\
1938 \\
1939 \\
1940\end{array}$ & $\begin{array}{l}832,949 \\
860,779 \\
862,635 \\
863,761 \\
866,595\end{array}$ & $\begin{array}{l}1,367,000 \\
1,411,900 \\
1,421,500 \\
1,422,000 \\
1,427,400\end{array}$ \\
\hline $\begin{array}{l}1941 \\
1942 \\
1943 \\
1944 \\
1945 \\
1946\end{array}$ & $\begin{array}{l}867,451 \\
871,673 \\
878,985 \\
881,803 \\
897,524 \\
918,340\end{array}$ & $\begin{array}{l}1,429,500 \\
1,444,900 \\
1,451,800 \\
1,455,400 \\
1,483,700 \\
1,519,300\end{array}$ \\
\hline
\end{tabular}




\section{SNAKE RIVER BASIN}

The Snake River is a major tributary of the Columbia River and has a drainage area of 109, 000 square miles. Rising in Yellowstone National Park in western Wyoming, it traverses southern Idaho westward in a broad arc and then flows almost due north to form a part of the boundaries between Idaho, Oregon, and Washington. Near Lewiston, Idaho it turns west to join the Columbia River near Pasco, Wash. The Snake River drains the southeastern part of the Columbia River basin including portions of the following States: Wyoming, Idaho, Utah, Nevada, Oregon, and Washington.

Mountain ranges and high plateaus enclose the Snake River basin. On the east are the Rockies, on the north are the Centennial Mountains and Beaverhead Range, to the west are the Blue Mountains and the high plateau of eastern Oregon, and to the south the barren mountains and plateaus of northern Nevadc. and Utah.

Within the Snake River basin lies one of the largest and most highly developed irrigated areas in the United States. The early settlers soon found the advantages of irrigation for crop production. The earlier irrigation projects were established by individuals who diverted the waters of small tributaries directly upon the land. As these smaller tributaries became fully utilized, large groups and companies were formed to draw upon the waters of the Snake River and its larger tributaries. Additional impetus was furnished by Federal legislation. These factors, combined with favorable natural conditions account for the remarkable growth which took place between 1890 and 1910. Recent development has been more gradual and generally limited to extension and intensified use of early projects, to provision of more storage facilities, or to evolution into multipurpose projects. From the early beginnings of nearly a century ago the irrigated area has grown until at present there are about $2,400,000$ acres irrigated abore Weiser, Idaho. In the pages that follow, each subdivision is treated in more detail.

\section{SNAKE RIVER ABOVE HEISE}

The drainage area above the gaging station on Snake River near Heise, Idaho, is about 5,700 square miles of which about 80 percent is in northwest Wyoming and the remainder is in east-central Idaho. The area is generally mountainous and its perimeter includes portions of the Continental Divide and of the Teton, Gros Ventre, Salt River and Caribou Ranges.

Snake River heads in Yellowstone National Park, thence flows southward about 65 miles before turning westward through the 
so-called Grand Canyon to the Wyoming-Idaho State line, and there veers generally northwest to and beyond Heise.

In Wyoming the main tributaries above Jackson Lake are the Lewis and Heart Rivers, which are fed primarily by geysers; those below this lake are the Buffalo Fork, Gros Ventre, Hoback, and Salt Rivers.

The drainage area is generally mountainous, with only a small portion suitable for agriculture and only a small fraction of this arable land is irrigated. Hay and forage crops predominate and are fed for the most part to livestock locally.

Irrigation in this area was developed primarily by private and cooperative enterprises, which had reached most of their growth before 1910. The principal diversions are from the Gros Ventre, Hoback, and Salt Rivers; minor amounts are diverted from the Snake River. There are three active storage reservcirs in the area with an aggregate capacity of 852,000 acre-feet, but very little of the stored water is used within the tributary kasin. All return flow from the irrigated area in this tributary basin is assumed to have returned to the Snake River above the ge.ging station at Heise. The net consumptive use was estimated to be 0.8 acre-feet per acre and was based upon computations by the LowryJohnson method. The irrigated area, estimated annual depletion and annual runoff for the gaging station at Heise are shown in the following table. The estimated depletion, expressed as a percentage of the yield of the watershed is 1.7 percent for the period 1921 through 1945. 
Irrigated area, annual runoff, and estimated depletion for Snake River above Haise, Idaho

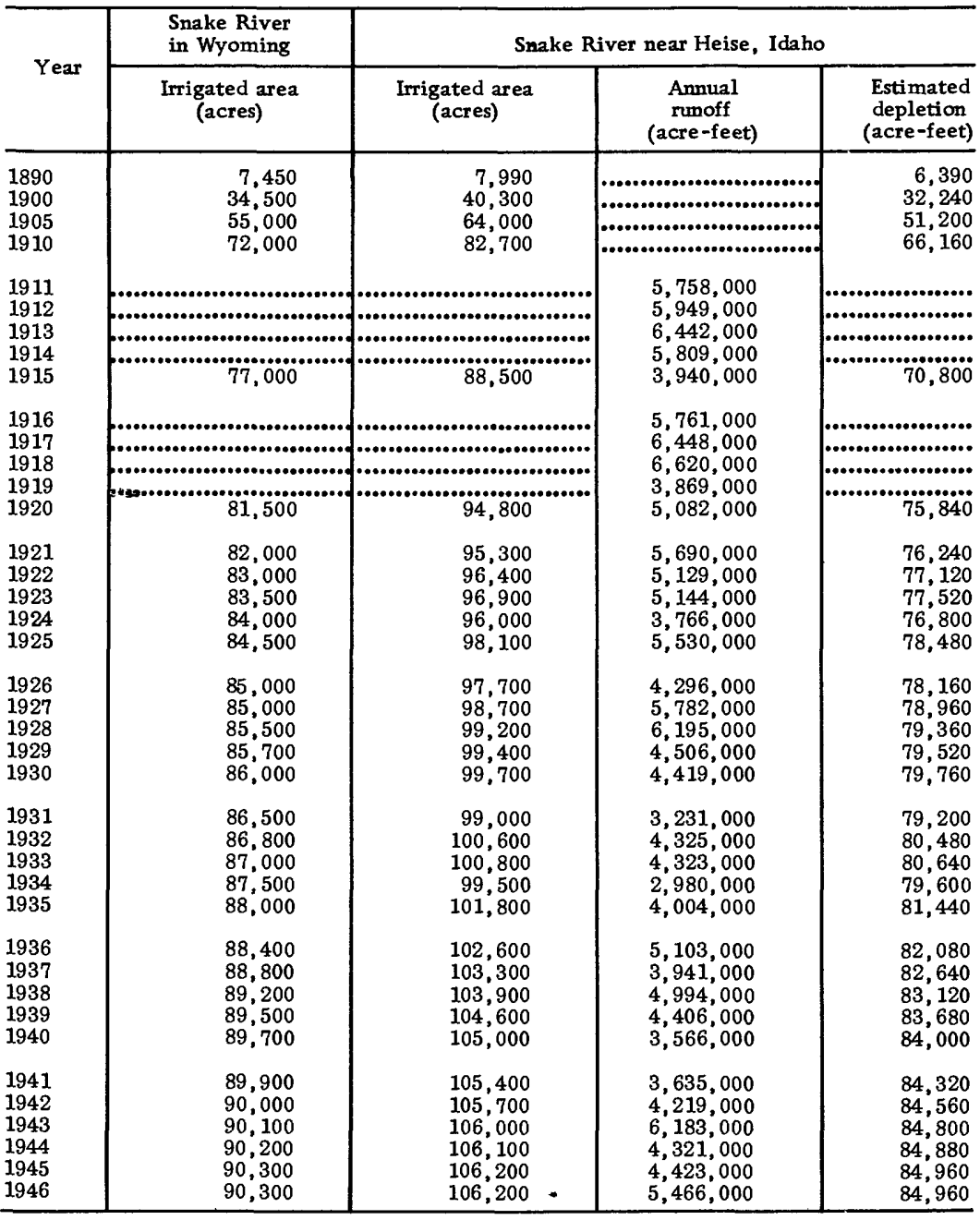

SNAKE RIVER, HEISE TO KING HILL

Along the main stem of Snake River from Heise to King Hill, lies one of the most intricate and highly developedirrigation systems in the United States. Most of the irrigated lands in this reach are in two compact bodies along either bank of the river.

In the upper reach--that is, from Heise. downstream tc American Falls irrigation was financed by private capital, excepting on the Fort Hall Indian Reservation lands. Canal companies, irrigation districts and water-user associations were organized to 
expand the irrigated area. Some of the earliest water rights in the upper Snake River basin exist here. Development was very rapid from 1890 to 1910 , with only a moderate subsequent expansion. Numerous canals serve this area; some of the larger ones are: Anderson, Eagle Rock, Harrison, Burgess, Butte, and Market Lake, Idaho, Snake River Valley, Great Western and Porter, Blackfoot, Peoples and Aberdeen-Springfield canals. Waters from Willow Creek and Blackfoot River are used interchangeably with water from Snake River in serving some of the lands. The Fort Hall irrigation system was developed for use by the Ind:an Service for lands under their jurisdiction.

In contrast to this area above American Falls, the lower area-from Lake Walcot to Bliss--largely was developed subsequent to 1900 as projects under the Carey Act and Bureau of Reclamation.

The South Side Twin Falls project is one of the largest and most successful of the Carey Act enterprises. It surrounds the towns of Twin Falls and Buhl south of the Snake River. Water for this area is diverted at Milner Dam, which has been in operation since 1905.

The North Side Twin Falls project is another very successful Carey Act enterprise. This is located north of the Srake River near Jerome and Bliss, opposite the South Side Twin Falls area. Water was first delivered during 1908 but the project was not substantially completed until 1916.

The Minidoka project, next downstream, was developed by the Bureau of Reclamation and includes three divisions - North Side Minidoka, South Side Minidoka, and Gooding. Part of this area has been turned over by the Bureau of Reclamation to irrigation districts. The development started as early as 1907 for the North and South Side divisions. The Gooding division was started in 1927, and delivered its first water to the lower Wood River area in 1931, thereby supplementing the water supply of that basin.

The netconsumptive use for this entire area between Heise and King Hill has been estımated to be 1.8 acre-feet per csere, based upon studies by the Bureau of Reclamation and others for several component projects. The return flow from all this area is assumed to be recaptured by the Snake River above the gaging station at King Hill, but in considerable part within a relatively short reach just upstream from that station.

\section{HENRYS FORK}

The Henrys Fork basin drains about 3,010 square miles of eastern Idaho and northwestern Wyoming. The Centennial Mountains, 
Teton and Snake River Ranges for $m$ the northern, eastern and southern boundaries.

Most of the irrigated lands are in the lower valley, chiefly between Ashton and Rexburg, although there are some irrigated meadows in the mountain valleys. The land in the lower valley was favorably situated and was among the first areas to be extensively developed in the upper Snake River basin. There are 36 canals diverting water from the Henrys Fork below Ashton and from the lower reaches of its larger tributaries. Of these the St. Anthony Union, Egin, Marysville, and Falls River Canals are the largest and on the average serve more than 8,000 acres each. The table on page 63 shows the estimated distribution $c$ the irrigated lands in this basin.

Owing to the porous nature of the soil, large quantities of water are applied. In some areas it is common practice to apply sufficient quantities to raise the water table to the root zone of the crops. However, the net consumptive use has been estimated 1.0 and 1.4 acre-feet per acre for the upper and lower portions of the basin respectively. In this report it is predicated that the proportionately large return flow from the irrigated area in the Henrys Fork basin is recaptured by the source streams only in small part, and in large part moves by way of the very large groundwater body which underlies the eastern and central parts of the Snake River Plain and which discharges principally in the many large springs in and near the Hagerman Valley upstream from King Hill. Accordingly, this report does not estimate depletion for the Henrys Fork and other subdivisions of the Snake River basin between Heise and King Hill. Depletion for all the area above King Hill is estimated in the table on page 70 . 
Irrigated area, in acres, at indicated years for Henrys Fork basin

\begin{tabular}{|c|c|c|c|}
\hline & $\begin{array}{c}\text { Henrys Fork } \\
\text { near Aston, Idaho }\end{array}$ & $\begin{array}{c}\text { Fall River } \\
\text { near Squirrel, Idaho }\end{array}$ & $\begin{array}{l}\text { Tetcn River above } \\
\text { St. Anthony, Idaho }\end{array}$ \\
\hline & $\begin{array}{l}\text { Irrigated area } \\
\text { (acres) }\end{array}$ & $\begin{array}{l}\text { Irrigated area } \\
\text { (acres) }\end{array}$ & $\begin{array}{l}\text { Irrigated area } \\
\text { (acres) }\end{array}$ \\
\hline $\begin{array}{l}1890 \\
1900\end{array}$ & $\begin{array}{r}960 \\
8,800\end{array}$ & 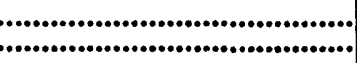 & $\begin{array}{r}920 \\
20,700\end{array}$ \\
\hline $\begin{array}{l}1905 \\
1910 \\
1915 \\
1920\end{array}$ & $\begin{array}{l}14,200 \\
17,100 \\
17,800 \\
18,100\end{array}$ & $\begin{array}{r}4,300 \\
6,500 \\
8,000 \\
10,000\end{array}$ & $\begin{array}{l}30,300 \\
34,300 \\
36,100 \\
37,100\end{array}$ \\
\hline $\begin{array}{l}1921 \\
1922 \\
1923 \\
1924 \\
1925\end{array}$ & $\begin{array}{l}18,200 \\
18,300 \\
18,400 \\
17,200 \\
18,500\end{array}$ & $\begin{array}{r}10,000 \\
10,200 \\
10,500 \\
8,500 \\
10,500\end{array}$ & $\begin{array}{l}37,300 \\
37,400 \\
37,500 \\
32,200 \\
37,700\end{array}$ \\
\hline $\begin{array}{l}1926 \\
1927 \\
1928 \\
1929 \\
1930\end{array}$ & $\begin{array}{l}18,000 \\
18,600 \\
18,600 \\
18,600 \\
18,600\end{array}$ & $\begin{array}{r}9,000 \\
10,500 \\
10,700 \\
11,000 \\
11,000\end{array}$ & $\begin{array}{l}34,200 \\
37,900 \\
38,000 \\
38,100 \\
38,200\end{array}$ \\
\hline $\begin{array}{l}1931 \\
1932 \\
1933 \\
1934 \\
1935\end{array}$ & $\begin{array}{l}16,500 \\
18,600 \\
18,600 \\
16,000 \\
18,200\end{array}$ & $\begin{array}{r}6,400 \\
10,000 \\
10,500 \\
8,000 \\
10,000\end{array}$ & $\begin{array}{l}33,200 \\
36,300 \\
38,300 \\
31,300 \\
37,300\end{array}$ \\
\hline $\begin{array}{l}1936 \\
1937 \\
1938 \\
1939 \\
1940\end{array}$ & $\begin{array}{l}18,600 \\
18,600 \\
18,600 \\
17,500 \\
17,500\end{array}$ & $\begin{array}{l}10,000 \\
12,200 \\
12,200 \\
13,200 \\
13,800\end{array}$ & $\begin{array}{l}38,300 \\
38,300 \\
38,300 \\
38,300 \\
38,300\end{array}$ \\
\hline $\begin{array}{l}1941 \\
1942 \\
1943 \\
1944 \\
1945 \\
1946\end{array}$ & $\begin{array}{l}17,500 \\
17,500 \\
17,500 \\
17,500 \\
17,500 \\
17,500\end{array}$ & $\begin{array}{l}13,800 \\
13,800 \\
14,000 \\
15,000 \\
15,500 \\
16,000\end{array}$ & $\begin{array}{l}38,300 \\
38,400 \\
38,400 \\
38,400 \\
38,400 \\
38,400\end{array}$ \\
\hline
\end{tabular}

\section{PORTNEUF RIVER}

The Portneuf River drains about 1,700 square miles south and southeast of Pocatello, Idaho. The stream heads on the Fort Hall Indian Reservation and flows south to the divide between the Snake and Bear Rivers; and then north to join the Snake River near Pocatello. 
The 33,700 irrigated acres in this basin are in two main areas-one just downstream from Portneuf Reservoir and the other near McCammon. These areas are under private management and are served by small ditches diverting water from tributary creeks and the main river. Hay and grains are the principal crops grown.

The growth of irrigated area within this tributary basin is shown in the following table. The net consumptive use for tre Portneuf River basin has been estimated at 1.6 acre-feet per acre. The return flow is believed to be through porous underbeds which drain towards the Snake River, and to sustain part of the discharge of the Portneuf springs. These springs are about 4 miles downstream from Pocatello.

MINOR TRIBUTARIES, NEELEY TO KING HILL

The minor tributaries joining the Snake River from the south, downstream from the Portneuf River, head in the barren plateaus which form the divide between the drainage basins of the Snake River and the Great Salt Lake. These tributaries are Paft River, Goose, Rock, and Salmon Falls Creeks. Most of the area irrigated by the water of these creeks lies along the south rim of the plain near Minidoka and South Side Twin Falls Projects. However there are scattered tracts along these creeks and near Rockland and Albion, Oakley and Kenyon, and Hollister and Amsterdam. Approximately 4,700 acres are in Utah and 11,500 acres in Nevada.

Net consumptive use for the lands irrigated by the tributaries has been estimated at 1.6 acre-feet per acre. Return flow has been assumed to be captured by the Snake River before it reaches King Hill. 


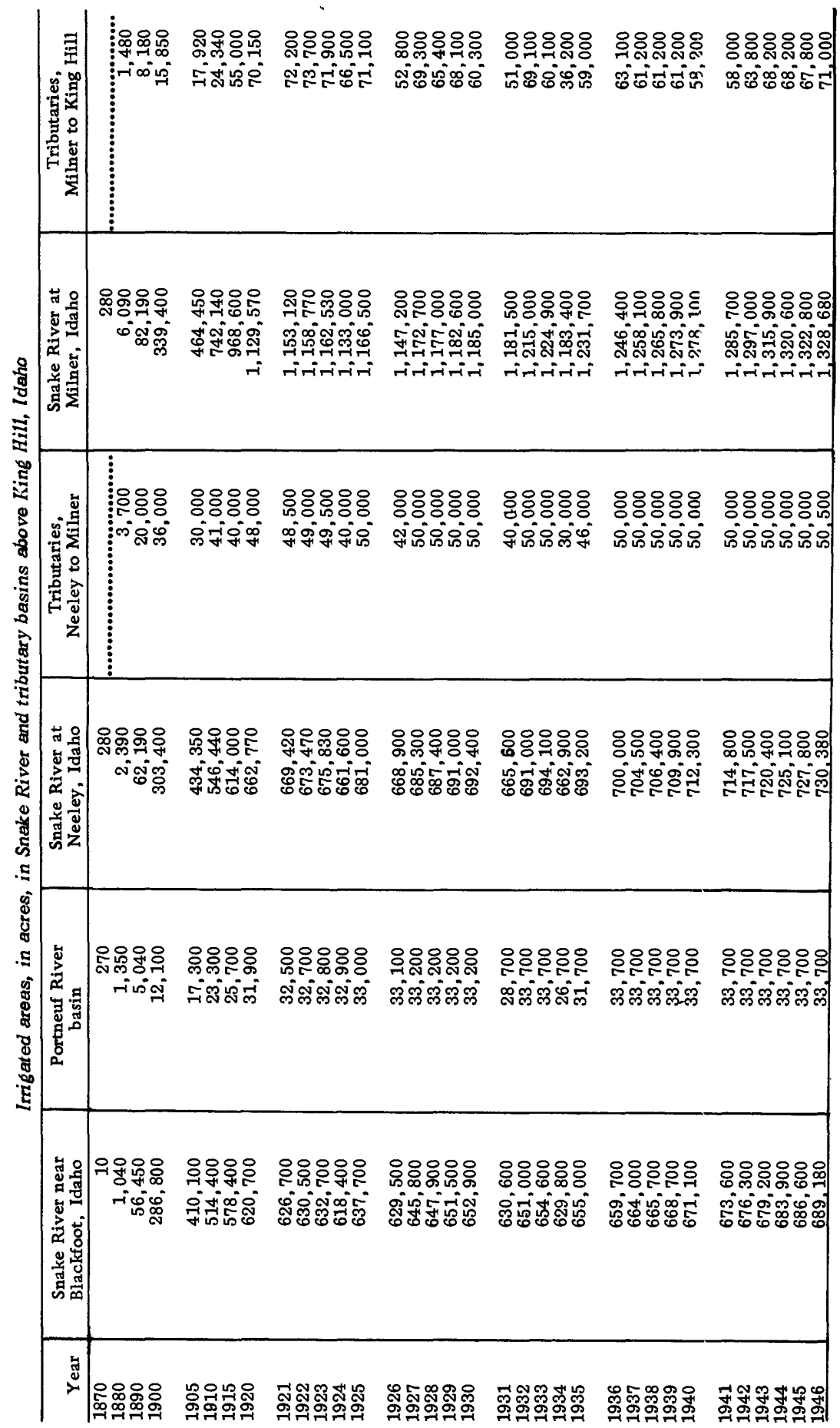

279538 O $-53-6$ 
MUD LAKE AREA

The Mud Lake area lies at the foot of the Beaverhead Range and Centennial Mountains of eastern Idaho and is adjacent to the Henrys Fork basin. It includes the basins of Camas, Beaver, Medicine Lodge, and Birch Creeks, and the adjacent lakes.

The irrigated land of this area is in the intermountain valleys along the several creeks just cited and around the south side of Mud Lake. Irrigation along the creeks previously mentioned began about 1870 in connection with stock ranches but it was not until 1908 that development using water from Mud Lake was started. At one time developments in this area were projected to include about 150,000 acres of which approximately 30,000 acres were to be in Carey Act projects. However at present time only about 47,000 are being irrigated. (See table on p. 69.) The water supply for these lands is largely pumped from Mud Lake and from adjacent lakes and sloughs and from shallow wells.

The net consumptive use on these lands has been estimated at 1.6 acre-feet per acre. Return flow is believed to be tributary to the ground-water body which sustains the Thousand Springs and other ground-water discharge in and near Hagerman Valley, far to the west.

\section{LITTLE LOST RIVER}

The Little Lost River basin is an intermontane valley between the Lemhi and Lost River Range and is just west of Rirch Creek. The river flows southward from these mountains and sinks into the north margin of the permeable Snake River Plain.

Settlement of the valley began about 1880; irrigaticn was introduced gradually until slightly over 11,000 acres wer? being irrigated by about 1910. Very little change in irrigated aroa has taken place subsequently except during extremely dry years when the available water has been insufficient for all lands. The irrigated area shown in the table on page 69 is mainly devoted to hay and forage crops and depends almost wholly upon the natural flow of the streams because there is no satisfactory storage of surplus water.

Net consumptive use for the lands of the Little Lost River basin has been estimated at 1.3 acre-feet per acre. Return flow has been assumed to be captured by the Snake River near Hagerman, far to the west. 
The Big Lost River drains about 2,000 square miles of central Idaho, between the Salmon and Snake Rivers. It heads in the Lost River Range and Sawtooth Mountains and flows southward to the margin of the Snake River Plain. Then it swings to the east and northeast and gradually "sinks" into the gravel-lined channel through which it flows.

As in adjacent basins, the agricultural lands are in the valleys near the mountains and are devoted largely to hay and other forage crops. Settlement began quite early, and by 1911 approximately 40,000 acres were being irrigated (see table on p. 69). Mackay Dam was constructed in 1916 and the reservoir now affords about 38,000 acre-feet of storage space. It was contemplated that this reservoir would be raised to a higher elevation but excessive leakage prevented this. By 1923 the irrigated area had increased to 50,000 acres, but during ensuing years of low runoff some lands could not be supplied with water.

The storage unit and water rights of the Utah Construction Co. were purchased by the Big Lost River Irrigation District prior to 1937. Thereafter the water previously applied to lands of the Carey Act project was used to supplement supplies available for the irrigation district lands. Currently about 42,000 acres, between Mackay Reservoir and Arco, are being irrigated.

Netconsumptive use has been estimated to be 1.3 acre-feet per acre. Return flow is assumed, like that of the Mud Lake area and the Little Lost River, to be captured by the Snake River just above King Hill.

\section{BIG WOOD RIVER}

The Big Wood River heads in the southern slopes of the Sawtooth Mountains of central Idaho and flows generally south and west to its confluence with the Snake River near Hagerman. Belor Henrys Fork, it is the first major tributary to join the Snake River from the north. Its basin is timbered in the upper reaches, kut in the lower reaches sage and cactus are the native vegetation.

The agricultural lands are in the upper valleys of the Pig Wood River and on the Snake River Plain. Hay and forage crops are dominate, although some diversified farming is practiced in the lower reaches.

Most of the irrigation projects in the Big Wood River basin were under the Carey Act but as such did not have the early rapid growth which characterized the areas further upstream. The water supply 
was plentiful in early years and overexpansion took place. This reached a climax about 1920-21, when approximately 117,000 acres were irrigated (see table on $p$. 69). The succession of dry years that followed 1921 showed the necessity of abantoning part of the acreage or developing a new source of water. To alleviate this condition, the Bureau of Reclamation in 1931 constructed the Milner-Gooding Canal, which diverts water from the Snake River at Milner, 75 miles to the southeast. This canal currently supplies water to 49,000 acres, of which 32,000 acres formerly were supplied from the Big Wood River.

The main irrigated areas extend from Ketchum to and below Bellevue, and center around Carey, Shoshone, Gooding, and Richfield. There is also some irrigated lands above Magic Reservoir in the Camas Creek basin and in the Fish Creek basin. This scattering of irrigated areas is due in part to excessively permeable soils which cover some portions of the sub-basin, and which cause excessive losses to ground water.

Net consumptive use for the lands of the Big Wood River basin has been estimated at 1.7 acre-feet per acre. Return flow has been assumed to reach the Snake River above King Hill, possibly via Malad Springs in whole or in part. 
Imigated area in acres for indicated years

\begin{tabular}{|c|c|c|c|c|c|}
\hline Year & Mud Lake basin & $\begin{array}{c}\text { Little Lost } \\
\text { River basin }\end{array}$ & $\begin{array}{l}\text { Big Lost River } \\
\text { above Mackay } \\
\text { Reservoir, Idaho }\end{array}$ & $\begin{array}{c}\text { Big Lost } \\
\text { River basin }\end{array}$ & $\begin{array}{l}\text { Big Wood } \\
\text { River basin }\end{array}$ \\
\hline $\begin{array}{l}1880 \\
1890 \\
1900\end{array}$ & $\begin{array}{r}10 \\
2,300 \\
9,200\end{array}$ & $\begin{array}{r}50 \\
1,540 \\
4,920\end{array}$ & $\begin{array}{r}130 \\
1,790 \\
5,100\end{array}$ & $\begin{array}{r}130 \\
1,790 \\
20,500\end{array}$ & $\begin{array}{r}14,810 \\
40,200\end{array}$ \\
\hline $\begin{array}{l}1905 \\
1910 \\
1915 \\
1920\end{array}$ & $\begin{array}{l}12,200 \\
13,300 \\
15,000 \\
26,200\end{array}$ & $\begin{array}{r}6,100 \\
7,900 \\
9,000 \\
11,400\end{array}$ & $\begin{array}{l}5,500 \\
8,300 \\
8,500 \\
8,000\end{array}$ & $\begin{array}{l}25,500 \\
38,300 \\
37,500 \\
42,000\end{array}$ & $\begin{array}{r}52,800 \\
71,400 \\
90,400 \\
115,100\end{array}$ \\
\hline $\begin{array}{l}1921 \\
1922 \\
1923 \\
1924 \\
1925\end{array}$ & $\begin{array}{l}27,400 \\
27,700 \\
28,900 \\
24,900 \\
27,600\end{array}$ & $\begin{array}{r}11,600 \\
11,800 \\
11,900 \\
9,200 \\
11,900\end{array}$ & $\begin{array}{r}9,000 \\
10,000 \\
10,000 \\
8,500 \\
9,500\end{array}$ & $\begin{array}{l}48,200 \\
49,700 \\
50,200 \\
43,800 \\
48,500\end{array}$ & $\begin{array}{l}117,000 \\
111,300 \\
113,200 \\
103,400 \\
111,900\end{array}$ \\
\hline $\begin{array}{l}1926 \\
1927 \\
1928 \\
1929 \\
1930\end{array}$ & $\begin{array}{l}24,800 \\
29,300 \\
29,900 \\
29,300 \\
29,600\end{array}$ & $\begin{array}{r}9,400 \\
11,900 \\
11,900 \\
11,900 \\
11,900\end{array}$ & $\begin{array}{l}8,700 \\
9,500 \\
9,500 \\
9,500 \\
9,200\end{array}$ & $\begin{array}{l}44,000 \\
46,500 \\
47,300 \\
47,300 \\
47,100\end{array}$ & $\begin{array}{l}108,700 \\
113,300 \\
110,800 \\
106,000 \\
101,300\end{array}$ \\
\hline $\begin{array}{l}1931 \\
1932 \\
1933 \\
1934 \\
1935\end{array}$ & $\begin{array}{l}24,000 \\
28,000 \\
30,000 \\
25,000 \\
31,000\end{array}$ & $\begin{array}{r}8,500 \\
11,900 \\
11,900 \\
8,000 \\
11,000\end{array}$ & $\begin{array}{l}8,000 \\
8,500 \\
8,700 \\
7,000 \\
8,000\end{array}$ & $\begin{array}{l}39,000 \\
43,500 \\
45,500 \\
33,000 \\
45,800\end{array}$ & $\begin{array}{l}74,500 \\
79,200 \\
82,200 \\
73,800 \\
77,700\end{array}$ \\
\hline $\begin{array}{l}1936 \\
1937 \\
1938 \\
1939 \\
1940\end{array}$ & $\begin{array}{l}34,000 \\
37,700 \\
39,200 \\
40,700 \\
41,500\end{array}$ & $\begin{array}{l}11,900 \\
11,900 \\
11,900 \\
11,900 \\
11,900\end{array}$ & $\begin{array}{l}9,000 \\
9,000 \\
9,000 \\
9,000 \\
9,000\end{array}$ & $\begin{array}{l}43,000 \\
42,000 \\
42,000 \\
42,000 \\
42,000\end{array}$ & $\begin{array}{l}81,900 \\
83,700 \\
82,900 \\
82,800 \\
83,100\end{array}$ \\
\hline $\begin{array}{l}1941 \\
1942 \\
1943 \\
1944 \\
1945 \\
1946\end{array}$ & $\begin{array}{l}42,000 \\
42,500 \\
42,700 \\
43,000 \\
43,200 \\
43,200\end{array}$ & $\begin{array}{l}11,900 \\
11,900 \\
11,900 \\
11,900 \\
11,900 \\
11,900\end{array}$ & $\begin{array}{l}9,000 \\
9,000 \\
9,000 \\
9,000 \\
9,000 \\
9,000\end{array}$ & $\begin{array}{l}42,000 \\
42,000 \\
42,000 \\
42,000 \\
42,000 \\
42,000\end{array}$ & $\begin{array}{l}83,100 \\
82,100 \\
85,100 \\
88,100 \\
88,600 \\
88,800\end{array}$ \\
\hline
\end{tabular}

* Beginning in 1931, a portion of lands formerly irrigated from Big Wood River was irrigated thereafter from Snake River through the Milner-Gooding Canal.

SNAKE RIVER AT KIING HILL, IDAHO

This is the first gaging station on the Snake River downstream from Heise for which an estimate depletion and yield would not be complicated by unmeasured flow bypassing the gaging station. The irrigated area shown in the following table is an accumulation of all irrigated area above this point. Likewise the estimated depletion is the summation of the depletion in each of the sub-basins above here. The estimated average depletion, during the period 1921 through 1945 represents 25.4 percent of the yield of the basin. Owing to the increase in irrigated area, the estimated depletion has gradually been increasing, and by 1946 it ropresented 27.7 percent of the long-term yield. The depletion due to irrigation between Heise and King Hill during the same period of time, represents 48.7 percent of the estimated yield of this subdivision. The estimated depletion for 1947 represents 53 percent of the long term yield of this subdivision. 
Irrigated area, annual runoff, and estimated depletion for Snake River at King Hill, Idaho

\begin{tabular}{|c|c|c|c|}
\hline Year & $\begin{array}{l}\text { Irrigated area } \\
\text { (acres) }\end{array}$ & $\begin{array}{c}\text { Runoff } \\
\text { (acre-f eet) }\end{array}$ & $\begin{array}{c}\text { Estimated } \\
\text { depletion } \\
\text { (acre-feet) }\end{array}$ \\
\hline $\begin{array}{l}1870 \\
1880 \\
1890 \\
1900\end{array}$ & $\begin{array}{r}280 \\
7,760 \\
117,710 \\
432,140\end{array}$ & 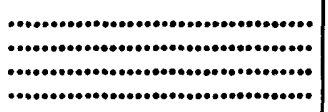 & $\begin{array}{r}450 \\
13,000 \\
176,000 \\
660,000\end{array}$ \\
\hline $\begin{array}{l}1905 \\
1910\end{array}$ & $\begin{array}{l}581,100 \\
899,740\end{array}$ & $9,370,000$ & $\begin{array}{r}878,000 \\
1,403,000\end{array}$ \\
\hline $\begin{array}{l}1911 \\
1912 \\
1913 \\
1914 \\
1915\end{array}$ & 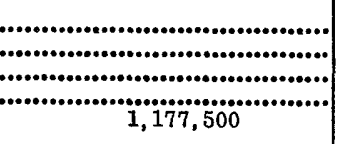 & $\begin{array}{r}8,940,000 \\
9,840,000 \\
10,400,000 \\
9,690,000 \\
7,420,000\end{array}$ & 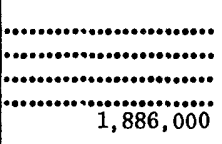 \\
\hline $\begin{array}{l}1916 \\
1917 \\
1918 \\
1919 \\
1920\end{array}$ & & $\begin{array}{r}8,990,000 \\
11,000,000 \\
9,610,000 \\
7,770,000 \\
7,100,000\end{array}$ & 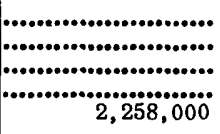 \\
\hline $\begin{array}{l}1921 \\
1922 \\
1923 \\
1924 \\
1925\end{array}$ & $\begin{array}{l}1,432,420 \\
1,435,870 \\
1,441,530 \\
1,382,800 \\
1,440,400\end{array}$ & $\begin{array}{l}9,470,000 \\
8,650,000 \\
7,970,000 \\
6,850,000 \\
8,260,000\end{array}$ & $\begin{array}{l}2,280,000 \\
2,320,000 \\
2,329,000 \\
2,242,000 \\
2,326,000\end{array}$ \\
\hline $\begin{array}{l}1926 \\
1927 \\
1928 \\
1929 \\
1930\end{array}$ & $\begin{array}{l}1,389,000 \\
1,445,900 \\
1,445,200 \\
1,448,100 \\
1,438,100\end{array}$ & $\begin{array}{l}7,240,000 \\
7,120,000 \\
9,440,000 \\
7,540,000 \\
6,690,000\end{array}$ & $\begin{array}{l}2,248,000 \\
2,336,000 \\
2,334,000 \\
2,339,000 \\
2,322,000\end{array}$ \\
\hline $\begin{array}{l}1931 \\
1932 \\
1933 \\
1934 \\
1935\end{array}$ & $\begin{array}{l}1,380,500 \\
1,449,600 \\
1,457,500 \\
1,361,200 \\
1,458,400\end{array}$ & $\begin{array}{l}5,880,000 \\
5,660,000 \\
5,820,000 \\
5,411,000 \\
5,070,000\end{array}$ & $\begin{array}{l}2,246,000 \\
2,348,000 \\
2,360,000 \\
2,219,000 \\
2,363,000\end{array}$ \\
\hline $\begin{array}{l}1936 \\
1937 \\
1938 \\
1939 \\
1940\end{array}$ & $\begin{array}{l}1,483,200 \\
1,497,500 \\
1,505,900 \\
1,515,400 \\
1,517,500\end{array}$ & $\begin{array}{l}6,209,000 \\
5,874,000 \\
7,233,000 \\
6,663,000 \\
5,687,000\end{array}$ & $\begin{array}{l}2,403,000 \\
2,354,000 \\
2,440,000 \\
2,456,000 \\
2,459,000\end{array}$ \\
\hline $\begin{array}{l}1941 \\
1942 \\
1943 \\
1944 \\
1945 \\
1946\end{array}$ & $\begin{array}{l}1,525,400 \\
1,542,100 \\
1,568,700 \\
1,576,700 \\
1,579,200 \\
1,588,480\end{array}$ & $\begin{array}{l}5,646,000 \\
6,434,000 \\
9,002,000 \\
7,592,000 \\
6,963,000 \\
8,684,000\end{array}$ & $\begin{array}{l}2,473,000 \\
2,502,000 \\
2,548,000 \\
2,561,000 \\
2,565,000 \\
2,581,000\end{array}$ \\
\hline
\end{tabular}

SNAKE RIVER, KING HILL TO MOUTH

\section{BRUNEAU RIVER}

The Bruneau River heads in the Copper Mountains of northern Nevada and drains about 3,200 square miles in that ste te and in southern Idaho. The terrain is moderately rugged and there is little agricultural enterprise other than stock raising. The small irrigated area in this basin, 39,000 acres is in scattered tracts along the main river and tributaries. (See the followirg table.) Except for that adjacent to the Snake River most of the irrigated area is hay and forage crops. 
The Grandview Irrigation District currently irrigates 5, 400 acres and is the largest single block of irrigated land in the basin. This tract is south of the Snake River downstream from the confluence of the Bruneau River; it uses water from both rivers. The net consumptive use has been estimated to be 1.8 acre-feet per acre. Return flow has beenassumed to be recaptured by the Snake River above the gaging station near Murphy.

Irrigated area and estimated depletion for Bruneau River basin

\begin{tabular}{|c|c|c|c|}
\hline \multirow{2}{*}{ Year } & \multirow{2}{*}{$\begin{array}{c}\begin{array}{c}\text { Brumeau River } \\
\text { near }\end{array} \\
\text { Hot Spring, Idaho } \\
\begin{array}{c}\text { Irrigated area } \\
\text { (acres) }\end{array}\end{array}$} & \multicolumn{2}{|c|}{ Bruneau River basin } \\
\hline & & $\begin{array}{l}\text { Irrigated area } \\
\text { (acres) }\end{array}$ & $\begin{array}{c}\text { Estimated } \\
\text { depletion } \\
\text { (acre-feet) }\end{array}$ \\
\hline $\begin{array}{l}1870 \\
1880 \\
1890 \\
1900\end{array}$ & $\begin{array}{c}2,600 \\
4,700\end{array}$ & $\begin{array}{r}18 \\
460 \\
4,590 \\
8,660\end{array}$ & $\begin{array}{r}30 \\
830 \\
8,260 \\
15,590\end{array}$ \\
\hline $\begin{array}{l}1905 \\
1910 \\
1915 \\
1920\end{array}$ & $\begin{array}{l}4,900 \\
5,220 \\
5,870 \\
6,100\end{array}$ & $\begin{array}{l}10,870 \\
14,430 \\
19,410 \\
19,810\end{array}$ & $\begin{array}{l}19,570 \\
25,970 \\
39,940 \\
35,660\end{array}$ \\
\hline $\begin{array}{l}1921 \\
1922 \\
1923 \\
1924 \\
1925\end{array}$ & $\begin{array}{l}6,290 \\
6,470 \\
6,650 \\
6,830 \\
7,020\end{array}$ & $\begin{array}{l}20,000 \\
20,690 \\
20,380 \\
20,580 \\
20,780\end{array}$ & $\begin{array}{l}36,000 \\
37,240 \\
36,680 \\
37,040 \\
37,400\end{array}$ \\
\hline $\begin{array}{l}1926 \\
1927 \\
1928 \\
1929 \\
1930\end{array}$ & $\begin{array}{l}7,200 \\
7,380 \\
7,560 \\
7,740 \\
7,820\end{array}$ & $\begin{array}{l}19,970 \\
20,610 \\
20,810 \\
20,460 \\
21,040\end{array}$ & $\begin{array}{l}35,950 \\
37,100 \\
37,460 \\
36,830 \\
37,870\end{array}$ \\
\hline $\begin{array}{l}1931 \\
1932 \\
1933 \\
1934 \\
1935\end{array}$ & $\begin{array}{l}7,890 \\
7,960 \\
8,040 \\
8,110 \\
8,180\end{array}$ & $\begin{array}{l}21,130 \\
21,220 \\
21,280 \\
21,360 \\
21,440\end{array}$ & $\begin{array}{l}38,030 \\
38,200 \\
38,300 \\
38,450 \\
38,590\end{array}$ \\
\hline $\begin{array}{l}1936 \\
1937 \\
1938 \\
1939 \\
1940\end{array}$ & $\begin{array}{l}8,260 \\
8,330 \\
8,410 \\
8,480 \\
8,480\end{array}$ & $\begin{array}{l}21,530 \\
21,700 \\
21,790 \\
21,860 \\
21,860\end{array}$ & $\begin{array}{l}38,750 \\
39,060 \\
39,220 \\
39,350 \\
39,350\end{array}$ \\
\hline $\begin{array}{l}1941 \\
1942 \\
1943 \\
1944 \\
1945 \\
1946\end{array}$ & $\begin{array}{l}8,480 \\
8,480 \\
8,480 \\
8,480 \\
8,480 \\
8,480\end{array}$ & $\begin{array}{l}21,860 \\
21,860 \\
21,860 \\
21,860 \\
21,860 \\
21,860\end{array}$ & $\begin{array}{l}39,350 \\
39,350 \\
39,350 \\
39,350 \\
39,350 \\
39,350 \\
\end{array}$ \\
\hline
\end{tabular}


SNAKE RIVER, KING HILL TO MURPHY

In addition to the area irrigated from Bruneau River there are about 17,000 irrigated acres within this subdivision. The miscellaneous creeks furnish most of the water for these lands. The area near Mountain Home is an exception. It receives part of its water supply from the South Fork Boise River drainage via a canal from Little Camas Reservoir to Long Tom Reservoir. This area was developed by private enterprise starting about 1895 , was consolidated in 1925 to the Mountain Home Irrigation District, and includes about 5,000 acres. However, only about 4,400 acres are currently being irrigated. These lands and the resulting streamflow depletions are included in this subdivision rather than under the Boise River basin. With the intermingling of water from two sources it becomes very difficult to segregate the acreage on the basis of the source of the irrigation water. Consequently in most cases the irrigated area and the estimated depletion of streamflow have been tabulated according to the geographic location, with cross-references to properly identify the areas involved.

The net consumptive use for all irrigated land in this subdivision was taken at 2.0 acre-feet per acre. Return flow from this area is assumed to be captured by the Snake River above the gaging station near Murphy.

The average streamflow depletion above the gaging station (see the following table) during the period 1921 through 1945 represents 25.5 percent of the yield of the basin. The irrigated area has been increasing slightly during the period and the estimated depletion for 1946 amounts to 27 percent of the long-term yield. In the subdivision between King Hill and Murphy the average depletion for the period 1921 through 1945 represents 18.2 percent of the yield of this subdivision, and it has remained essentially constant during the base period. 
Irrigated area, annual runoff, and estimated depletion for Snake River neav Murphy, Idaho

\begin{tabular}{|c|c|c|c|}
\hline Year & $\begin{array}{l}\text { Irrigated area } \\
\text { (acres) }\end{array}$ & $\begin{array}{c}\text { Runoff } \\
\text { (acre-feet) }\end{array}$ & $\begin{array}{l}\text { Estimated } \\
\text { depletion } \\
\text { (acre-feet) }\end{array}$ \\
\hline $\begin{array}{l}1870 \\
1880 \\
1890 \\
1900\end{array}$ & $\begin{array}{r}1,000 \\
12,280 \\
129,470 \\
451,400\end{array}$ & \multirow{4}{*}{ 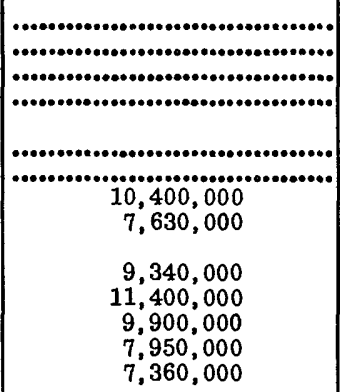 } & \multirow{5}{*}{$\begin{array}{r}1,890 \\
21,930 \\
198,600 \\
696,790 \\
919,220 \\
1,453,700 \\
1,959,400 \\
\\
\ldots \ldots \ldots \ldots \ldots \ldots \ldots \ldots \ldots \\
\ldots \ldots \ldots \ldots \ldots \ldots \ldots \\
\ldots \ldots \ldots \ldots \ldots \ldots \\
2,338,900 \\
2,361,400 \\
2,401,800 \\
2,409,700 \\
2,322,900 \\
2,404,850\end{array}$} \\
\hline 1905 & $\begin{array}{l}602,800 \\
926,520\end{array}$ & & \\
\hline $\begin{array}{l}1914 \\
1915\end{array}$ & $1,216,100$ & & \\
\hline $\begin{array}{l}1916 \\
1917 \\
1918 \\
1919 \\
1920\end{array}$ & 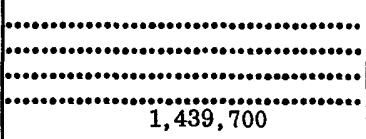 & & \\
\hline $\begin{array}{l}1921 \\
1922 \\
1923 \\
1924 \\
1925\end{array}$ & $\begin{array}{l}1,475,100 \\
1,478,900 \\
1,483,900 \\
1,425,300 \\
1,481,900\end{array}$ & $\begin{array}{l}9,970,000 \\
9,020,000 \\
8,250,000 \\
7,090,000 \\
8,630,000\end{array}$ & \\
\hline $\begin{array}{l}1926 \\
1927 \\
1928 \\
1929 \\
1930\end{array}$ & $\begin{array}{l}1,429,100 \\
1,486,300 \\
1,485,800 \\
1,488,300 \\
1,478,400\end{array}$ & $\begin{array}{l}7,480,000 \\
7,430,000 \\
9,760,000 \\
7,790,000 \\
6,940,000\end{array}$ & $\begin{array}{l}2,324,200 \\
2,412,600 \\
2,411,000 \\
2,415,400 \\
2,398,500\end{array}$ \\
\hline $\begin{array}{l}1931 \\
1932 \\
1933 \\
1934 \\
1935\end{array}$ & $\begin{array}{l}1,420,700 \\
1,490,200 \\
1,497,600 \\
1,401,000 \\
1,497,700\end{array}$ & $\begin{array}{l}6,110,000 \\
6,120,000 \\
6,020,000 \\
5,523,000 \\
5,337,000\end{array}$ & $\begin{array}{l}2,322,110 \\
2,424,900 \\
2,436,000 \\
2,294,300 \\
2,437,200\end{array}$ \\
\hline $\begin{array}{l}1936 \\
1937 \\
1938 \\
1939 \\
1940\end{array}$ & $\begin{array}{l}1,522,200 \\
1,536,900 \\
1,545,900 \\
1,555,500 \\
1,557,600\end{array}$ & $\begin{array}{l}6,682,000 \\
6,105,000 \\
7,690,000 \\
6,979,000 \\
5,988,000\end{array}$ & $\begin{array}{l}2,476,700 \\
2,428,400 \\
2,515,600 \\
2,531,700 \\
2,534,700\end{array}$ \\
\hline $\begin{array}{l}1941 \\
1942 \\
1943 \\
1944 \\
1945 \\
1946\end{array}$ & $\begin{array}{l}1,565,400 \\
1,581,700 \\
1,608,400 \\
1,615,800 \\
1,618,200 \\
1,627,400\end{array}$ & $\begin{array}{l}5,910,000 \\
6,870,000 \\
9,788,000 \\
8,051,000 \\
7,452,000 \\
9,043,000\end{array}$ & $\begin{array}{l}2,548,700 \\
2,576,800 \\
2,623,100 \\
2,634,800 \\
2,638,700 \\
2,654,600 \\
\end{array}$ \\
\hline
\end{tabular}

\section{OWYHEE RIVER}

The Owyhee River is the principal tributary of the Snake River from the south, and drains about 12,000 square miles. It heads in the mountains of northern Nevada and flows generally northwest and north across southwestern Idaho and southeastern Oregon toits confluence with the Snake River just upstream from the Boise River. The drainage area is primarily rough to rolling terrain, and has a native cover almost exclusively of sagebrush.

The irrigated lands are located in three general a reas -- the Western Shoshone Indian Reservation in Nevada, the valley of Jordan Creek in Idaho and Oregon, and the Owyhee. Project in Oregon. Other irrigated areas are scattered throughort the basin. 
Above Owyhee Reservoir stock raising is the principal agricultural enterprise and most of the irrigated land is in hay or forage crops. In contrast, the area below Owyhee Dam is primarily devoted to row and specialty crops.

In large part the irrigated lands in the upper basin are within the Shoshone Indian Reservation; these have been irrigated gradually by the Bureau of Indian Affairs. Wild Horse Reservoir in Nevada, on the South Fork Owyhee River, was completed in 1937 and furnishes water for about 9,200 acres of land in the Western Shoshone Indian Reservation. This reservoir has an active capacity of 32,000 acre-feet. Other irrigated tracts in this portion of the basin are scattered along the South Fork and East Fork of the Owyhee River.

The Jordan Valley Irrigation District was formed in 1926 by consolidating some of the old water rights of that valley in Oregon. Antelope Reservoir, an off-stream storage development, supplies water for the 8,000 acres in this district. In addition, about 18,000 acres of irrigated land within the Jordan Creek basin is irrigated from natural streamflow.

The largest and most productive block of irrigated land is 1ocated below Owyhee Dam and comprises about 105, 000 acres in eight operating divisions. Of this about 32,000 to $3 \$, 000$ acres are estimated to be within the natural drainage basin of the Malheur River. Earlier projects irrigated by the Owyhee Canal, Gem Irrigation District, Shoestring Ditch, Kingman Colony Irrigation Co. and the Riverview Irrigation System have been integrated into the Owyhee Project or are being supplied with supplemental water by it. Of these only the Owyhee Canal originally diverted water from Owyhee River; the others pumped their water from the Snake River. The Gem Irrigation District is designe- to utilize water from either the Snake or the Owyhee Rivers, and since 1937 has drawn from both those sources. In this district the water from Owyhee Reservoir has been used to irrigate new lands while that pumped from the Snake River has served the old lands.

The irrigated areas shown in the following table are based upon adjudicated rights, Water-Supply Paper 657, and reports and records of Bureau of Census, Bureau of Reclamation, irrigation districts, and irrigation companies. Data from these several sources were adjusted to common values, which are believed to be reasonably dependable. 
Net consumptive use values of 1.2 and 1.9 acre-feet per acre were used for lands above and below Owyhee Reservoir, respectively. Return flow from all lands in Nevada was assumed to reach the East Fork or South Fork within that State; that from lands in Jordan Creek valley and above Owyhee Reservioir, to be recaptured above the gaging stations in those two subcivisions. Return flow from the lands served by the Owyhee project was assumed to be captured by the Snake River above the gaging station at Weiser. The average streamflow depletion for the portion of the sub-basin above Owyhee Reservoir during the period 1931 through 1946 amounts to 13 percent of the yield of the basin. The irrigated area below Owyhee Reservoir has been expanding rapidly during the period and consequently the average depletion over the period would have little meaning. The average depletion for 1945-46 for the entire basin amounts to 27 percent of the longterm yield of the gaging station above Owyhee Reservoir. 


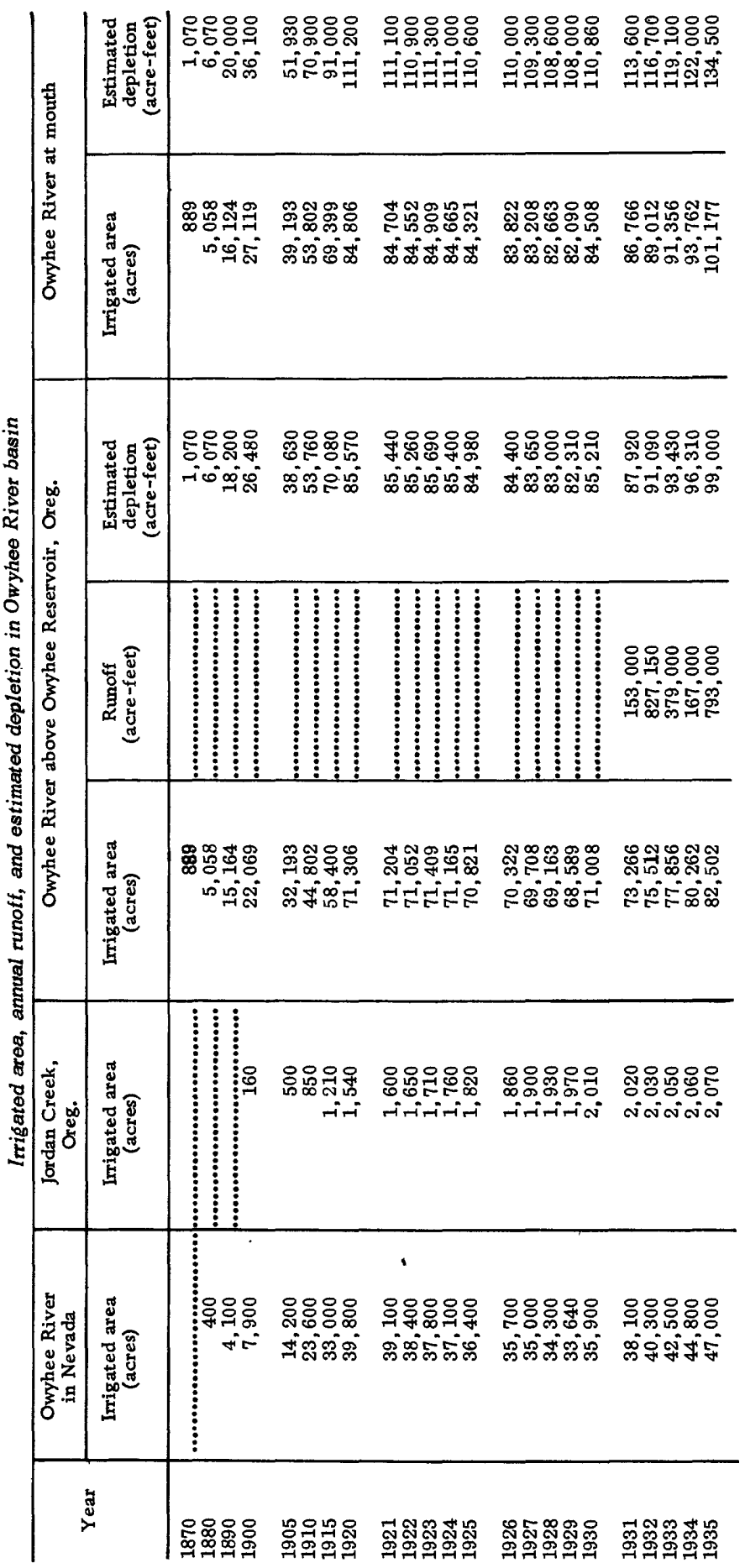


궁유윰 윰융유

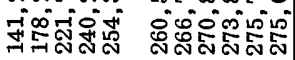

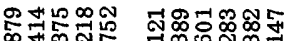

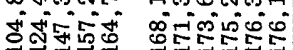

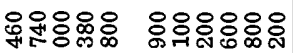

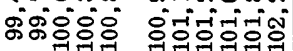

위융유유 융융ํํ유

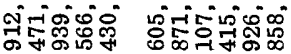

-i

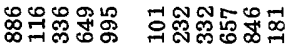

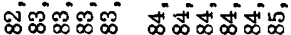

ㅇㅇㅇ유유 융유유

niñona ninanañ

유윰융 양유유윰

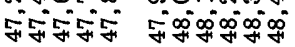

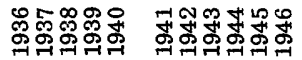




\section{BOISE RIVER}

The Boise River rises in the Sawtooth Mountains of central Idaho and flows generally westward to its confluence with the Snake River near Parma. It drains about 4,100 square miles of which only about 30 percent are below an altitude of 5,000 feet. Its mountainous area is generally forest covered and fairly rugged. The lower valley contains a highly developed block of irrigated land extending from Boise west and southwest to the Snake River.

Some of the earliest water rights in the Snake River basin are in the Boise valley. According to the "Steward Decree" the se extend back to 1864. Early development was by private enterprise, but recent development has been primarily sponsored by the $\mathrm{Bu}-$ reau of Reclamation, which started work in this basin in 1905 and up to the present has continued to assist in irrigation development. The Arrowrock division of the Boise Project furnishes full or supplemental water supplies for most of the irrigated lands in the basin. (See tables on pp. 80 and 81.)

The acreage irrigated in the headwater and tributar basins of the Boise River was computed from data in court decrees, State water licenses, and census reports. The Boise River watermaster has very complete reports for the main valley for eacl year, beginning about 1915; extensive use was made of these reports and of reports by the U. S. Bureau of Reclamation. Accurate figures were available from surveys relating to litigation in 1903 and the census of 1902. Reliable data for several of the larger irrigation ditches for 1911 and 1912 were obtained from reports of the State Engineer. Reports of the U. S. Bureau of Reclamatior list acreages for others for each year back to 1906 . The relation between actual irrigated area and decreed water rights for the ditches with accurate records, was applied to the rest of the mcre than $\mathbf{5 0}$ ditches, estimates of irrigated area were made that are believed to be reliable for the years since about 1902 .

The amount of land irrigated prior to 1902 is subject to considerable doubt. All water rights are known from State records, and the earliest dates from 1865. At first it was possible to establish a water right as soon as one-eighth of the land was in cultivation. Many ditch companies were slow to cultivate all the land for which they had water rights. Thus it was impossible to derive reliable estimates of irrigated area from data on water-right decrees. Reports of the State Engineer gave estimates of irrigated acreage for 1896 and 1900. Acreages for all intervening periods were obtained by interpolation. In the lower valley the system of canc.ls, lateral and drains is so complex that only the total acreage has any significance. 
Considerable information on the principal Boise River canals is given in Water-Supply Paper 657, and on all canals in the annual reports of the watermaster for the Boise River. The tabulated figures for the Boise River include a small acreage in Oregon lying east of the Snake River opposite Adrian, Oreg., and irrigated from the Boise River.

Water is diverted from the South Fork Boise River for irrigation of land near Mountain Home, which was previously described. This irrigated area and the resulting depletion was not included in the totals for Boise River.

Diversified crops are generally grown throughout the basin at present, although in early days most of the irrigated land was devoted to grain and forage and hay crops. Considerable land in the lower areas are waterlogged and drainage has become a major problem locally.

The net consumptive use was taken as 1.0 and 2.2 acr?-feet per acre for the upper and lower portions of the Boise River basin respectively. The value for the lower basin was based upon the inflow-outflow studies by Steward and Paul on the Pioneer and Nampa-Meridian Irrigation Districts in 1917. The return flow is recaptured by the Boise and Snake Rivers and is included in the flow passing the gaging station on Snake River at Weiser. In some parts of the lower valley the return flow from upstream areas is reused during the late summer. The average depletion in the upper basin is negligible. The average depletion for entire basin for the period 1921 through 1945 represents 28.6 percent of the yield of the Boise River basin above Dowling Ranch plus Moore Creek. The depletion has been increasing slightly during recent years and for 1946 it was 29.3 percent of the long-term yield. 
Irrigated area, annual runoff and estimated depletion in the upper Boise River basin

\begin{tabular}{|c|c|c|c|c|c|}
\hline \multirow{2}{*}{ Year } & \multirow{2}{*}{\begin{tabular}{|c|}
$\begin{array}{c}\text { South Fork Boise } \\
\text { River near } \\
\text { Featherville, Idaho }\end{array}$ \\
$\begin{array}{c}\text { Irrigated area } \\
\text { (acres) }\end{array}$ \\
\end{tabular}} & \multirow{2}{*}{$\begin{array}{c}\begin{array}{c}\text { South Fork Boise } \\
\text { River at Anderson } \\
\text { Ranch Dam, Idaho }\end{array} \\
\begin{array}{c}\text { Irrigated area } \\
\text { (acres) }\end{array}\end{array}$} & \multicolumn{3}{|c|}{$\begin{array}{c}\text { Boise River at Dowling Ranch } \\
\text { near Arrowrock, Idaho }\end{array}$} \\
\hline & & & $\begin{array}{l}\text { Irrigated area } \\
\text { (acres) }\end{array}$ & $\begin{array}{c}\text { Runoff } \\
\text { (acre-feet }\end{array}$ & $\begin{array}{c}\text { Estimated } \\
\text { depletion } \\
\text { (acre-feet) }\end{array}$ \\
\hline $\begin{array}{l}1890 \\
1900 \\
1905 \\
1910\end{array}$ & $\begin{array}{l}110 \\
160 \\
170\end{array}$ & $\begin{array}{l}170 \\
650 \\
630 \\
650\end{array}$ & $\begin{array}{r}170 \\
960 \\
1,760 \\
2,520\end{array}$ & 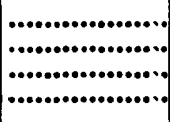 & $\begin{array}{r}170 \\
960 \\
1,760 \\
2,520\end{array}$ \\
\hline $\begin{array}{l}1912 \\
1913\end{array}$ & & & & $\begin{array}{l}2,080,000 \\
1,790,000\end{array}$ & - \\
\hline 1914 & & & & $1,890,000$ & \\
\hline 1915 & 250 & 810 & 3,100 & $1,040,000$ & 3,100 \\
\hline $\begin{array}{l}1916 \\
1917\end{array}$ & & & & $\begin{array}{l}2,190,000 \\
2,070,000\end{array}$ & \\
\hline $\begin{array}{l}1918 \\
1919\end{array}$ & & & & $\begin{array}{l}1,740,000 \\
1,470,000\end{array}$ & •.e...... \\
\hline 1920 & 490 & 1,320 & 3,710 & $1,360,000$ & 3,710 \\
\hline $\begin{array}{l}1921 \\
1922 \\
1923 \\
1924 \\
1925\end{array}$ & $\begin{array}{l}550 \\
600 \\
650 \\
700 \\
740\end{array}$ & $\begin{array}{l}1,440 \\
1,540 \\
1,640 \\
1,740 \\
1,840\end{array}$ & $\begin{array}{l}3,790 \\
3,860 \\
3,930 \\
4,000 \\
4,070\end{array}$ & $\begin{array}{r}2,500,000 \\
2,070,000 \\
1,560,000 \\
827,000 \\
2,040,000\end{array}$ & $\begin{array}{l}3,790 \\
3,860 \\
3,930 \\
4,000 \\
4,070\end{array}$ \\
\hline $\begin{array}{l}1926 \\
1927 \\
1928 \\
1929 \\
1930\end{array}$ & $\begin{array}{l}750 \\
750 \\
750 \\
750 \\
750\end{array}$ & $\begin{array}{l}1,840 \\
1,840 \\
1,850 \\
1,850 \\
1,850\end{array}$ & $\begin{array}{l}4,040 \\
4,020 \\
3,990 \\
3,960 \\
3,930\end{array}$ & $\begin{array}{r}999,000 \\
2,270,000 \\
2,120,000 \\
1,190,000 \\
1,200,000\end{array}$ & $\begin{array}{l}4,040 \\
4,020 \\
3,990 \\
3,960 \\
3,930\end{array}$ \\
\hline $\begin{array}{l}1931 \\
1932 \\
1933 \\
1934 \\
1935\end{array}$ & $\begin{array}{l}750 \\
750 \\
750 \\
750 \\
750\end{array}$ & $\begin{array}{l}1,850 \\
1,850 \\
1,850 \\
1,850 \\
1,850\end{array}$ & $\begin{array}{l}3,930 \\
3,930 \\
3,930 \\
3,930 \\
3,930\end{array}$ & $\begin{array}{r}861,000 \\
1,700,000 \\
1,430,000 \\
962,500 \\
1,408,000\end{array}$ & $\begin{array}{l}3,930 \\
3,930 \\
3,930 \\
3,930 \\
3,930\end{array}$ \\
\hline $\begin{array}{l}1936 \\
1937 \\
1938 \\
1939 \\
1940\end{array}$ & $\begin{array}{l}750 \\
750 \\
750 \\
750 \\
750\end{array}$ & $\begin{array}{l}1,850 \\
1,850 \\
1,850 \\
1,850 \\
1,850\end{array}$ & $\begin{array}{l}3,930 \\
3,930 \\
3,930 \\
3,930 \\
3,930\end{array}$ & $\begin{array}{l}1,737,00 C \\
1,015,00 C \\
2,254,00 C \\
1,226,00 C \\
1,386,00 C\end{array}$ & $\begin{array}{l}3,930 \\
3,930 \\
3,930 \\
3,930 \\
3,930\end{array}$ \\
\hline $\begin{array}{l}1941 \\
1942 \\
1943 \\
1944 \\
1945 \\
1946\end{array}$ & $\begin{array}{l}750 \\
750 \\
750 \\
750 \\
750 \\
750\end{array}$ & $\begin{array}{l}1,850 \\
1,850 \\
1,850 \\
1,850 \\
1,850 \\
1,850\end{array}$ & $\begin{array}{l}3,930 \\
3,930 \\
3,930 \\
3,930 \\
3,930 \\
3,930\end{array}$ & $\begin{array}{l}1,261,00 \mathrm{C} \\
1,499,00 \mathrm{C} \\
3,006,00 \mathrm{C} \\
1,198,00 \mathrm{C} \\
1,449,00 \mathrm{C} \\
2,025,00 \mathrm{C}\end{array}$ & $\begin{array}{l}3,930 \\
3,930 \\
3,930 \\
3,930 \\
3,930 \\
3,930\end{array}$ \\
\hline
\end{tabular}


Irrigated area and estimated depletion in the Boise River basin

\begin{tabular}{|c|c|c|c|}
\hline \multirow{2}{*}{ Year } & $\begin{array}{c}\text { Moore Creek } \\
\text { near } \\
\text { Arrowrock, Idaho }\end{array}$ & \multicolumn{2}{|c|}{ Boise River basin } \\
\hline & $\begin{array}{l}\text { Irrigated area } \\
\text { (acres) }\end{array}$ & $\begin{array}{l}\text { Imigated area } \\
\text { (acres) }\end{array}$ & $\begin{array}{l}\text { Estimated } \\
\text { depletion } \\
\text { (acre-feet) }\end{array}$ \\
\hline $\begin{array}{l}1870 \\
1880 \\
1890 \\
1900\end{array}$ & 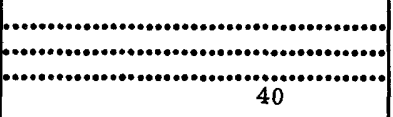 & $\begin{array}{r}6,000 \\
16,000 \\
26,000 \\
56,000\end{array}$ & $\begin{array}{r}13,200 \\
35,200 \\
57,200 \\
122,000\end{array}$ \\
\hline $\begin{array}{l}1905 \\
1910 \\
1915 \\
1920\end{array}$ & $\begin{array}{l}150 \\
290 \\
510 \\
680\end{array}$ & $\begin{array}{l}122,590 \\
173,435 \\
255,384 \\
295,776\end{array}$ & $\begin{array}{l}267,320 \\
378,180 \\
557,510 \\
645,440\end{array}$ \\
\hline $\begin{array}{l}1921 \\
1922 \\
1923 \\
1924 \\
1925\end{array}$ & $\begin{array}{l}690 \\
700 \\
700 \\
710 \\
720\end{array}$ & $\begin{array}{l}295,979 \\
295,143 \\
295,314 \\
305,142 \\
304,934\end{array}$ & $\begin{array}{l}645,780 \\
643,840 \\
644,130 \\
665,660 \\
665,110\end{array}$ \\
\hline $\begin{array}{l}1926 \\
1927 \\
1928 \\
1929 \\
1930\end{array}$ & $\begin{array}{l}720 \\
730 \\
740 \\
750 \\
750\end{array}$ & $\begin{array}{l}319,870 \\
329,808 \\
330,774 \\
334,684 \\
337,122\end{array}$ & $\begin{array}{l}698,000 \\
719,880 \\
722,030 \\
730,650 \\
736,05 r\end{array}$ \\
\hline $\begin{array}{l}1931 \\
1932 \\
1933 \\
1934 \\
1935\end{array}$ & $\begin{array}{l}760 \\
770 \\
780 \\
790 \\
800\end{array}$ & $\begin{array}{l}337,494 \\
329,472 \\
337,380 \\
329,232 \\
328,127\end{array}$ & $\begin{array}{l}736,860 \\
719,200 \\
736,580 \\
718,650 \\
716,200\end{array}$ \\
\hline $\begin{array}{l}1936 \\
1937 \\
1938 \\
1939 \\
1940\end{array}$ & $\begin{array}{l}800 \\
810 \\
820 \\
830 \\
840\end{array}$ & $\begin{array}{l}329,265 \\
332,199 \\
331,154 \\
331,497 \\
337,166\end{array}$ & $\begin{array}{l}718,710 \\
725,150 \\
722,840 \\
723,580 \\
736,040\end{array}$ \\
\hline $\begin{array}{l}1941 \\
1942 \\
1943 \\
1944 \\
1945 \\
1946\end{array}$ & $\begin{array}{l}850 \\
860 \\
870 \\
890 \\
900 \\
910\end{array}$ & $\begin{array}{l}334,088 \\
333,615 \\
331,122 \\
335,610 \\
335,081 \\
334,329\end{array}$ & $\begin{array}{l}729,260 \\
728,210 \\
722,710 \\
732,560 \\
731,380 \\
729,720\end{array}$ \\
\hline
\end{tabular}

\section{MALHEUR RIVER}

The Malheur River rises in Strawberry Mountain of eastern Oregon and flows eastward to its confluence with the Sncke River near Ontario, Oreg. It has a drainage area of about 4,700 square miles, of which the higher parts are sparsely covered by coniferous trees, and the lower parts originally were covered by desert vegetation.

The Malheur River valley was known to the early explorers and fur trader's and is reputed to have been named by Peter Skene Ogden, a Hudson's Bay Co. trader. Early settlers who were headed for the Oregon coast, sometimes traversed it via Bend to Salem and Portland. It was natural that some of the earliest pioneers settled in this broadvalley and took up farming, raising stock and 
forage crops, which were the most profitable ventures of the day. With increase in population specialty crops and orchards have become the important products of the region.

Most of the early irrigation projects were financed by private interests and some of the early canals have rights dating back to 1881-1885. These canals include the McLaughlin, Neveda, Linebarger, Gellerman and Froman, Farmer, J. H., Canyon and Sand Hollow, Mill, Vines, and Brosman. The possibilities of expanding irrigation in this valley have been investigated on seviral occasions by Federal and State agencies, most intensively in 19031905, 1914 and 1927. During the early investigations an association of water users was formed by some of the irrigators in the valley but satisfactory arrangements could not be reached between this association and the Reclamation Service.

Following the investigations of 1914, the Warmsprings Irrigation District was formed, by consolidating some of the existing irrigation units. This district constructed the Warm Springs Reservoir in 1919 to a capacity of 170,000 acre-feet. In 1930 the capacity was increased to 190,000 acre-feet and half of this was sold to the Bureau of Reclamation to serve the Vale project. In return the Bureau of Reclamation constructed a series of drain ditches throughout the lands of the Warmsprings Irrigation District to reclaim some lands which had been abandoned because of waterlogging. Later, in 1935, the Bureau built the Agency Valley Reservoir on the North Fork Malheur River for use of the Vale project. The Vale project is currently serving about 31,000 acrss and the Warmsprings Irrigation District about 18,000 acres.

The Willow Creek No. 3 dam was constructed in $1910-11$ by the Willow River Land and Irrigation Co, as a part of the Brogan project. The promoters expected to irrigate a large area around and below the town of Brogan in the lower valley of Willow Creek, and the reservoir was constructed with a capacity of 50,800 acre-feet as the major storage supply for the project lands. Discharge records for Willow Creek were not available at the time, except for a part of the flood of 1910 . This record was extremely misleading as it covered a period during one of the greatest floods in the stream's history. The reservoir has never filled. An irrigation distribution system was constructed to serve about 25,010 acres, a large part of the land was sold to prospective settlers, and scattered tracts were irrigated. However after much litigation the project was reduced to about 3,000 acres, for which the water supply is still inadequate. 
The remainder of the irrigated area shown in the following table is scattered throughout the basin, primarily in connention with ranches on the upper tributaries.

The net consumptive use for this basin has been estirr ated to be 1.0 acre-foot per acre for the South Fork and main stem, 1.6 acre-feet per acre for the North Fork and the middle reaches of Willow Creek, and 1.9 acre-feet per acre for the lower basin including Bully Creek. The return flow from the lands irrigated above Drewsey all returns to the main stem above the gaging station near that place; there is no irrigation and no additional return flow between this point and the Warmsprings Reservoir.

There is no gaging station in the lower valley that can be used for a depletion evaluation, inasmuch as all stations are complicated by ungaged flow bypassing them.

In addition to the lands irrigated from the Malheur River and tributaries, a large block of land is supplied from the Owyhee River. The return.flow from this and from all lands in the lower valley is assumed to be captured by the Snake River above the gaging station at Weiser, Idaho. 
Irrigated area and estimated depletion for Malheur River basin

\begin{tabular}{|c|c|c|c|}
\hline \multirow{2}{*}{ Year } & \multirow{2}{*}{$\begin{array}{c}\text { Malheur River } \\
\text { below Warm Springs } \\
\text { Reservoir, Oreg. } \\
\begin{array}{c}\text { Irrigated area } \\
\text { (acres) }\end{array}\end{array}$} & \multicolumn{2}{|c|}{ Malheur River basin } \\
\hline & & $\begin{array}{l}\text { Irrigated area } \\
\text { (acres) }\end{array}$ & $\begin{array}{l}\text { Estimated } \\
\text { depletion } \\
\text { (acre-feet) }\end{array}$ \\
\hline $\begin{array}{l}1870 \\
1880 \\
1890 \\
1900\end{array}$ & $\begin{array}{r}46 \\
3,573 \\
7,191\end{array}$ & $\begin{array}{r}196 \\
2,019 \\
28,099 \\
35,521\end{array}$ & $\begin{array}{r}300 \\
3,300 \\
46,500 \\
56,600\end{array}$ \\
\hline $\begin{array}{l}1905 \\
1910 \\
1915 \\
1920\end{array}$ & $\begin{array}{r}8,156 \\
8,905 \\
10,205 \\
12,137\end{array}$ & $\begin{array}{l}40,687 \\
45,055 \\
48,532 \\
52,016\end{array}$ & $\begin{array}{l}64,000 \\
70,900 \\
75,700 \\
80,200\end{array}$ \\
\hline $\begin{array}{l}1921 \\
1922 \\
1923 \\
1924 \\
1925\end{array}$ & $\begin{array}{l}12,153 \\
12,283 \\
12,284 \\
12,284 \\
12,284\end{array}$ & $\begin{array}{l}53,312 \\
53,799 \\
56,079 \\
56,208 \\
57,599\end{array}$ & $\begin{array}{l}82,600 \\
83,400 \\
87,800 \\
88,000 \\
90,600\end{array}$ \\
\hline $\begin{array}{l}1926 \\
1927 \\
1928 \\
1929 \\
1930\end{array}$ & $\begin{array}{l}12,284 \\
12,284 \\
12,284 \\
12,363 \\
12,456\end{array}$ & $\begin{array}{l}59,339 \\
63,106 \\
63,134 \\
63,217 \\
64,624\end{array}$ & $\begin{array}{r}93,900 \\
100,100 \\
100,200 \\
100,300 \\
102,800\end{array}$ \\
\hline $\begin{array}{l}1931 \\
1932 \\
1933 \\
1934 \\
1935\end{array}$ & $\begin{array}{l}12,501 \\
12,501 \\
12,501 \\
12,501 \\
12,501\end{array}$ & $\begin{array}{l}65,261 \\
67,440 \\
67,613 \\
68,842 \\
70,210\end{array}$ & $\begin{array}{l}103,900 \\
108,000 \\
108,300 \\
110,600 \\
113,200\end{array}$ \\
\hline $\begin{array}{l}1936 \\
1937 \\
1938 \\
1939 \\
1940\end{array}$ & $\begin{array}{l}12,561 \\
12,561 \\
12,561 \\
12,561 \\
12,561\end{array}$ & $\begin{array}{l}72,955 \\
77,102 \\
80,622 \\
82,085 \\
83,557\end{array}$ & $\begin{array}{l}118,400 \\
126,300 \\
133,000 \\
135,800 \\
138,600\end{array}$ \\
\hline $\begin{array}{l}1941 \\
1942 \\
1943 \\
1944 \\
1945 \\
1946\end{array}$ & $\begin{array}{l}12,561 \\
12,561 \\
12,561 \\
12,583 \\
12,583 \\
12,743\end{array}$ & $\begin{array}{l}83,910 \\
86,522 \\
88,195 \\
88,652 \\
89,090 \\
89,716\end{array}$ & $\begin{array}{l}139,220 \\
144,200 \\
147,400 \\
148,200 \\
149,000 \\
150,100 \\
\end{array}$ \\
\hline
\end{tabular}

\section{PAYETTE RIVER}

The Payette River drains about 3, 300 square miles, including some of the high mountainous terrain of central Idah?. Its confluence with the Snake River is near Ontario, Oreg. The upper basin, above Black Canyon Dam, is mountainous and ir large part timbered. Most agricultural lands are in the lower kasin on the valley floor from Black Canyon Dam westward to the Srake River.

For the upper basin, irrigated acreages were computed from water-right decrees, reports of irrigation districts, and the census reports. In the lower basin most of the irrigation enterprises are cooperative ditch companies, and water is distrikuted on the basis of shares rather than acreage, thus reliable data was scarce. 
Accurate records are available for the Emmett Irrigaticn District and Black Canyon unit No. 2, both of which divert water at Black Canyon Dam, but for the lands further downstream it was necessary to interpolate on basis of the meager data available. For 1900 the acreage was obtained from the report of the State Engineer, for 1902 from the census report, for 1938 from a report by the Bureau of Reclamation, and for 1939 from the census report. The Geological Survey report "Storage and Power Possibilities, Payette River basin, Idaho" by W. G. Hoyt, dated Janue ry, 1927, and later incorporated in Water-Supply Paper No. 657, stated that the irrigated area below Black Canyon Dam was betweon 80, 000 and 85,000 acres. Since most of the field work for that report was done during 1925 , it was assumed that the figures applied to that year and 82,000 acres was adopted for this study. The census figure for 1919 appeared to be rather large, so it was assumed that the ratio between actual irrigation and that reported was the same as for the Boise River. This gave a figure consistent with known information.

From George Knowles, engineer and ex-officio watermaster, it was learned that the increase in acreage was gradual between 1902 and 1919, but that later a large amount of land was abandoned because of waterlogging. He reported that construction of drain ditches was started in 1929 and that since about 1940 some 10,000 acres had been returned to irrigation. The above information, the Bureau of Reclamation report of 1938, and the 1939 irrigation census were sufficient to establish accurately the irrig ted acreage after 1938. For the intervening years back to 1902 the acreage was interpolated between the few computed values. The second annual report of the U. S. Reclamation Service (1902-03) stated that practically all development had been made during the preceding 10 years. On this basis, the irrigated acreage prior to 1900 was computed back to the beginning in 1865. (See tables on pp. 86, 87.) Over 800,000 acre-feet of storage capacity has been developed in four major reservoirs in the upper basin. These are the Payette Lake, Little Payette Lake, Deadwood, and Cascade Reservoirs. It is ironic that in this, one of the most intensely irrigated areas in the State, records of irrigated acreage should be so fragmentary. Because of the abundance of water, the water rights have never been adjudicated and many users have not bothered to register their water rights.

The net consumptive use for lands in this basin have been estimated at 1.0 acre-feet per acre for those above Bleck Canyon Dam and at 2.2 acre-feet per acre for all lands below that dam. The return flow is assumed to be recaptured by Payette and Snake Rivers, and all of it passes the gaging station on Snate River at Weiser. The average depletion for the upper basin during the period 1921 through 1945 amounts to 2.1 percent of the yield of 
the basin as measured at the gaging station near Horseshoe Bend. The streamflow depletion above here has remained essentially constant during the period. The average depletion for the entire basin for the same period, amounts to 10.2 percent of the yield of the basin at the gaging station near Horseshoe Berid. Because additional lands are being irrigated, the depletion has been increasing during recent years and in 1946 amounted to 13.2 percent of the long-term yield.

Irrigated area in upper Payette River basin

\begin{tabular}{|c|c|c|c|c|}
\hline Year & $\begin{array}{c}\text { South Fork Payette } \\
\text { River near } \\
\text { Banks, Idaho }\end{array}$ & $\begin{array}{c}\text { Lake Fork } \\
\text { below Lake } \\
\text { Irrigation District } \\
\text { canal, near } \\
\text { McCall, Idaho } \\
\end{array}$ & $\begin{array}{l}\text { North Fork } \\
\text { Payette R. } \\
\text { at Cascade, } \\
\text { Idaho }\end{array}$ & $\begin{array}{c}\text { North Fork Payette } \\
\text { River near } \\
\text { Banks, Idaho* }\end{array}$ \\
\hline $\begin{array}{l}1890 \\
1900\end{array}$ & $\begin{array}{l}1,880 \\
2,770\end{array}$ & 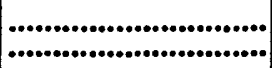 & 3,000 & $\begin{array}{r}390 \\
3,700\end{array}$ \\
\hline $\begin{array}{l}1905 \\
1910 \\
1915 \\
1920\end{array}$ & $\begin{array}{l}2,770 \\
2,770 \\
2,780 \\
2,820\end{array}$ & {$\left[\begin{array}{c}\ldots \\
\cdots\end{array}\right]$} & $\begin{array}{r}5,000 \\
7,600 \\
10,400 \\
14,000\end{array}$ & $\begin{array}{r}6,100 \\
9,300 \\
12,700 \\
17,200\end{array}$ \\
\hline $\begin{array}{l}1921 \\
1922 \\
1923 \\
1924 \\
1925\end{array}$ & $\begin{array}{l}2,830 \\
2,840 \\
2,850 \\
2,870 \\
2,880\end{array}$ & 3,000 & $\begin{array}{l}16,200 \\
18,100 \\
23,600 \\
25,900 \\
32,500\end{array}$ & $\begin{array}{l}19,900 \\
22,200 \\
28,900 \\
31,800 \\
39,800\end{array}$ \\
\hline $\begin{array}{l}1926 \\
1927 \\
1928 \\
1929 \\
1930\end{array}$ & $\begin{array}{l}2,880 \\
2,890 \\
2,900 \\
2,900 \\
2,910\end{array}$ & $\begin{array}{l}5,000 \\
5,000 \\
5,500 \\
6,000 \\
6,000\end{array}$ & $\begin{array}{l}35,000 \\
35,300 \\
36,500 \\
38,100 \\
38,100\end{array}$ & $\begin{array}{l}43,000 \\
43,300 \\
44,700 \\
46,700 \\
46,700\end{array}$ \\
\hline $\begin{array}{l}1931 \\
1932 \\
1933 \\
1934 \\
1935\end{array}$ & $\begin{array}{l}2,910 \\
2,910 \\
2,910 \\
2,910 \\
2,910\end{array}$ & $\begin{array}{l}6,000 \\
6,000 \\
6,000 \\
6,000 \\
6,000\end{array}$ & $\begin{array}{l}35,900 \\
35,900 \\
36,600 \\
36,800 \\
37,000\end{array}$ & $\begin{array}{l}44,000 \\
44,000 \\
44,900 \\
45,100 \\
45,400\end{array}$ \\
\hline $\begin{array}{l}1936 \\
1937 \\
1938 \\
1939 \\
1940\end{array}$ & $\begin{array}{l}2,910 \\
2,910 \\
2,910 \\
2,910 \\
2,910\end{array}$ & $\begin{array}{l}6,000 \\
6,000 \\
6,000 \\
6,000 \\
6,000\end{array}$ & $\begin{array}{l}36,000 \\
36,000 \\
36,800 \\
36,820 \\
36,800\end{array}$ & $\begin{array}{l}44,000 \\
44,000 \\
45,100 \\
45,140 \\
45,100\end{array}$ \\
\hline $\begin{array}{l}1941 \\
1942 \\
1943 \\
1944 \\
1945 \\
1946\end{array}$ & $\begin{array}{l}2,910 \\
2,910 \\
2,910 \\
2,910 \\
2,910 \\
2,910\end{array}$ & $\begin{array}{l}6,000 \\
6,000 \\
6,000 \\
6,000 \\
6,000 \\
6,000\end{array}$ & $\begin{array}{l}36,800 \\
36,800 \\
36,800 \\
37,200 \\
37,200 \\
37,400\end{array}$ & $\begin{array}{l}45,100 \\
45,100 \\
45,100 \\
45,700 \\
45,700 \\
45,900\end{array}$ \\
\hline
\end{tabular}

- This figure also represents irrigation above gage on North Fork Payette River near Smiths Ferry, Idaho, (discontinued) and total for North Fork Payette River. 
Irrigated area, annual runoff, and estimated depletion for the Payette River basin

\begin{tabular}{|c|c|c|c|c|c|}
\hline \multirow[b]{2}{*}{ Year } & \multicolumn{3}{|c|}{ Payette River near Horseshoe Bend, Idaho } & \multicolumn{2}{|c|}{ Payette River at mouth } \\
\hline & $\begin{array}{l}\text { Irrigated area } \\
\text { (acres) }\end{array}$ & $\begin{array}{c}\text { Runoff } \\
\text { (acre-feet) }\end{array}$ & $\begin{array}{l}\text { Estimated } \\
\text { depletion } \\
\text { (acre-feet) }\end{array}$ & $\begin{array}{l}\text { Irrigated area } \\
\text { (acres) }\end{array}$ & $\begin{array}{c}\text { Estimated } \\
\text { depletion } \\
\text { (acre-feet) }\end{array}$ \\
\hline $\begin{array}{l}1870 \\
1880 \\
1890 \\
1900\end{array}$ & $\begin{array}{r}40 \\
860 \\
3,140 \\
7,380\end{array}$ & 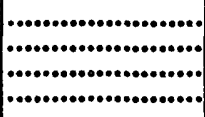 & $\begin{array}{r}40 \\
860 \\
3,140 \\
7,380\end{array}$ & $\begin{array}{r}1,650 \\
5,890 \\
15,050 \\
39,350\end{array}$ & $\begin{array}{r}3,090 \\
9,730 \\
25,850 \\
72,950\end{array}$ \\
\hline $\begin{array}{l}1905 \\
1908\end{array}$ & 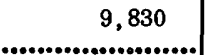 & $\because 2,170,000$ & 9,830 & 56,980 & 107,980 \\
\hline 1909 & & $3,050,000$ & & & 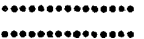 \\
\hline 1910 & 13,160 & $3,160,000$ & 13,160 & 73,180 & 139,180 \\
\hline $\begin{array}{l}1911 \\
1912\end{array}$ & & $\begin{array}{l}2,860,000 \\
2,540,000\end{array}$ & & & \\
\hline 1913 & & $2,510,000$ & . & & \\
\hline $\begin{array}{l}1915 \\
1920\end{array}$ & $\begin{array}{l}16,650 \\
21,190\end{array}$ & $\begin{array}{l}1,560,000 \\
1,840,000\end{array}$ & $\begin{array}{l}16,650 \\
21,190\end{array}$ & $\begin{array}{r}92,010 \\
109,950\end{array}$ & $\begin{array}{l}176,370 \\
210,390\end{array}$ \\
\hline $\begin{array}{l}1921 \\
1922 \\
1923 \\
1924 \\
1925\end{array}$ & $\begin{array}{l}23,900 \\
26,200 \\
32,920 \\
35,840 \\
43,850\end{array}$ & $\begin{array}{l}3,250,000 \\
2,390,000 \\
2,110,000 \\
1,110,000 \\
2,450,000\end{array}$ & $\begin{array}{l}23,900 \\
26,200 \\
32,920 \\
35,840 \\
43,850\end{array}$ & $\begin{array}{l}113,760 \\
115,270 \\
121,680 \\
123,700 \\
130,910\end{array}$ & $\begin{array}{l}215,520 \\
216,070 \\
222,120 \\
227,240 \\
229,310\end{array}$ \\
\hline $\begin{array}{l}1926 \\
1927 \\
1928 \\
1929 \\
1930\end{array}$ & $\begin{array}{l}47,050 \\
47,360 \\
48,770 \\
50,780 \\
50,780\end{array}$ & $\begin{array}{l}1,290,000 \\
3,140,000 \\
2,880,000 \\
1,440,000 \\
1,560,000\end{array}$ & $\begin{array}{l}47,050 \\
47,360 \\
48,770 \\
50,780 \\
50,780\end{array}$ & $\begin{array}{l}131,620 \\
130,420 \\
130,330 \\
130,840 \\
129,840\end{array}$ & $\begin{array}{l}227,020 \\
224,020 \\
222,130 \\
220,840 \\
218,640\end{array}$ \\
\hline $\begin{array}{l}1931 \\
1932 \\
1933 \\
1934 \\
1935\end{array}$ & $\begin{array}{l}48,080 \\
48,080 \\
48,980 \\
49,180 \\
49,479\end{array}$ & $\begin{array}{l}1,060,000 \\
2,290,000 \\
1,960,000 \\
1,635,000 \\
1,654,000\end{array}$ & $\begin{array}{l}48,080 \\
48,080 \\
48,980 \\
49,180 \\
49,479\end{array}$ & $\begin{array}{l}127,160 \\
127,000 \\
128,350 \\
129,050 \\
130,760\end{array}$ & $\begin{array}{l}215,980 \\
215,630 \\
217,520 \\
218,820 \\
222,220\end{array}$ \\
\hline $\begin{array}{l}1936 \\
1937 \\
1938 \\
1939 \\
1940\end{array}$ & $\begin{array}{l}48,090 \\
48,110 \\
49,228 \\
49,280 \\
49,260\end{array}$ & $\begin{array}{l}2,103,000 \\
1,364,000 \\
2,859,000 \\
1,608,000 \\
2,192,000\end{array}$ & $\begin{array}{l}48,090 \\
48,110 \\
49,228 \\
49,280 \\
49,260\end{array}$ & $\begin{array}{l}129,360 \\
129,690 \\
131,320 \\
133,700 \\
139,190\end{array}$ & $\begin{array}{l}220,800 \\
221,490 \\
223,720 \\
228,880 \\
240,970\end{array}$ \\
\hline $\begin{array}{l}1941 \\
1942 \\
1943 \\
1944 \\
1945 \\
1946\end{array}$ & $\begin{array}{l}49,280 \\
49,300 \\
49,320 \\
49,930 \\
49,950 \\
50,150\end{array}$ & $\begin{array}{l}1,938,000 \\
2,283,000 \\
3,478,000 \\
1,465,000 \\
1,958,000 \\
2,644,000\end{array}$ & $\begin{array}{l}49,280 \\
49,300 \\
49,320 \\
49,930 \\
49,950 \\
50,150\end{array}$ & $\begin{array}{l}143,150 \\
148,470 \\
156,040 \\
161,280 \\
164,710 \\
167,610\end{array}$ & $\begin{array}{l}249,650 \\
261,320 \\
277,950 \\
288,720 \\
296,140 \\
302,370\end{array}$ \\
\hline
\end{tabular}


WEISER RIVER

The Weiser River is the last downstream tributary entering the right bank of the Snake River before that stream flows through another canyon section. Thus the Weiser River subdivision is the downstream or nor thernmost segment of the intensively irrigated area in the Snake River basin. It drains about 1,600 scuare miles. The mountainous part of the basin is generally forested. The valleys and adjacent bench land constitute that portion of the basin which is devoted to agriculture. The irrigated tracts are small, scattered throughout the basin, and were developed by private capital. Very little storage capacity is available in the basin, and many of the projects would benefit from supplemental water late in the growing season: The irrigated lands are concentrated generally in three main areas -- an upper area near Council, near Mesa, and on Hornet Creek; a middle area near Cambridge and Midvale; and a lower area including adjacent benches along the Snake River near Weiser.

In general, the values of irrigated acreage for this subdivision basin were based upon water-right adjudications, reports of irrigation districts, Idaho State Engineer, Bureau of Reclamation, and Bureau of the Census; also on Water-Supply Paper 657. A Bureau of Reclamation report gave substantially higher figures than the 1939 Census andwere given more weight in the determination of values for this report. (See tables on pp. 8:, 90.)

In this report, the estimated net consumptive use within the Weiser River basin was 1.5 acre-feet per acre for the area above the gaging station on Weiser River above Crane Creek, and 2.0 acre-feet per acre for the area below Crane Creek. The return flow from the area above the gaging station, Weiser River above Crane Creek, was assumed to have been recaptured by the river above that station. That from the area below Crane Creek was assumed, for convenience, to have been intercepted ky the Snake River above the gaging station at Weiser. This may not be strictly true because some of the return flow from the irrigated bench lands near Weiser may reach the Snake River below the gage. However its effect on the Snake River flow would b? relatively minor. The average streamflow depletion during the period 1922 through 1946 amounts to 5 percent of the yield of the basin above the gaging station Weiser River near Crane Creek. It has remained comparatively constant during the period. 
Irrigated area in upper Weiser River basin, Idaho

[Note: No irrigation above gages on Weiser River st Tamarack, Idaho, and Lost Creek near Tamarack, Idaho]

\begin{tabular}{|c|c|c|c|c|}
\hline Year & $\begin{array}{c}\text { Weiser River } \\
\text { at } \\
\text { Starkey, Idaho }\end{array}$ & $\begin{array}{c}\text { Weiser River } \\
\text { near } \\
\text { Council, Idaho }\end{array}$ & $\begin{array}{c}\text { Weiser River } \\
\text { near } \\
\text { Cambridge, Idaho }\end{array}$ & $\begin{array}{l}\text { Little Weiser } \\
\text { River near } \\
\text { Indian Valley, } \\
\text { Idaho }\end{array}$ \\
\hline $\begin{array}{l}1880 \\
1890 \\
1900\end{array}$ & $\begin{array}{r}7 \\
72\end{array}$ & $\begin{array}{r}18 \\
1,370 \\
3,240\end{array}$ & $\begin{array}{r}18 \\
1,570 \\
3,610\end{array}$ & $\begin{array}{r}48 \\
80\end{array}$ \\
\hline $\begin{array}{l}1905 \\
1910 \\
1915 \\
1920\end{array}$ & $\begin{array}{l}72 \\
72 \\
72 \\
72\end{array}$ & $\begin{array}{l}4,670 \\
6,410 \\
7,050 \\
7,050\end{array}$ & $\begin{array}{l}5,170 \\
7,100 \\
8,080 \\
8,400\end{array}$ & $\begin{array}{l}80 \\
80 \\
80 \\
80\end{array}$ \\
\hline $\begin{array}{l}1921 \\
1922 \\
1923 \\
1924 \\
1925\end{array}$ & $\begin{array}{l}72 \\
72 \\
72 \\
72 \\
72\end{array}$ & $\begin{array}{l}7,050 \\
7,050 \\
7,050 \\
7,050 \\
7,050\end{array}$ & $\begin{array}{l}8,460 \\
8,510 \\
8,570 \\
8,630 \\
8,690\end{array}$ & $\begin{array}{l}80 \\
80 \\
80 \\
80 \\
80\end{array}$ \\
\hline $\begin{array}{l}1926 \\
1927 \\
1928 \\
1929 \\
1930\end{array}$ & $\begin{array}{l}72 \\
72 \\
72 \\
72 \\
72\end{array}$ & $\begin{array}{l}7,050 \\
7,050 \\
7,050 \\
7,050 \\
7,050\end{array}$ & $\begin{array}{l}8,750 \\
8,810 \\
8,870 \\
8,920 \\
8,980\end{array}$ & $\begin{array}{l}80 \\
80 \\
80 \\
80 \\
80\end{array}$ \\
\hline $\begin{array}{l}1931 \\
1932 \\
1933 \\
1934 \\
1935\end{array}$ & $\begin{array}{l}72 \\
72 \\
72 \\
72 \\
72\end{array}$ & $\begin{array}{l}7,050 \\
7,050 \\
7,050 \\
7,050 \\
7,050\end{array}$ & $\begin{array}{l}9,040 \\
9,100 \\
9,160 \\
9,220 \\
9,220\end{array}$ & $\begin{array}{l}80 \\
80 \\
80 \\
80 \\
80\end{array}$ \\
\hline $\begin{array}{l}1936 \\
1937 \\
1938 \\
1939 \\
1940\end{array}$ & $\begin{array}{l}72 \\
72 \\
72 \\
72 \\
72\end{array}$ & $\begin{array}{l}7,050 \\
7,050 \\
7,050 \\
7,050 \\
7,050\end{array}$ & $\begin{array}{l}9,220 \\
9,220 \\
9,220 \\
9,220 \\
9,220\end{array}$ & $\begin{array}{l}80 \\
80 \\
80 \\
80 \\
80\end{array}$ \\
\hline $\begin{array}{l}1941 \\
1942 \\
1943 \\
1944 \\
1945 \\
1946\end{array}$ & $\begin{array}{l}72 \\
72 \\
72 \\
72 \\
72 \\
72\end{array}$ & $\begin{array}{l}7,050 \\
7,050 \\
7,050 \\
7,050 \\
7,050 \\
7,050\end{array}$ & $\begin{array}{l}9,220 \\
9,220 \\
9,220 \\
9,220 \\
9,220 \\
9,220\end{array}$ & $\begin{array}{l}80 \\
80 \\
80 \\
80 \\
80 \\
80 \\
\end{array}$ \\
\hline
\end{tabular}


Irrigated area, annual runoff, and estimated depletion for Weiser River basin

\begin{tabular}{|c|c|c|c|c|c|}
\hline \multirow[b]{2}{*}{ Year } & \multicolumn{3}{|c|}{ Weiser River above Crane Creek near Weiser, Idaho } & \multicolumn{2}{|c|}{ Weiser River at mouth* } \\
\hline & $\begin{array}{l}\text { Irrigated area } \\
\text { (acres) }\end{array}$ & $\begin{array}{c}\text { Rumoff } \\
\text { (acrerfeet) }\end{array}$ & $\begin{array}{l}\text { Estimated } \\
\text { depletion } \\
\text { (acre-feet) }\end{array}$ & $\begin{array}{l}\text { Irrigated area } \\
\text { (acres) }\end{array}$ & $\begin{array}{l}\text { Estimated } \\
\text { depletion } \\
\text { (acre-feet) }\end{array}$ \\
\hline $\begin{array}{l}1870 \\
1880 \\
1890 \\
1900\end{array}$ & $\begin{array}{r}480 \\
7,900 \\
15,000\end{array}$ & 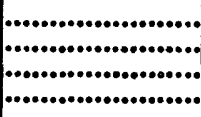 & $\begin{array}{r}720 \\
11,850 \\
22,500\end{array}$ & $\begin{array}{r}32 \\
1,340 \\
12,820 \\
23,350\end{array}$ & $\begin{array}{r}60 \\
2,440 \\
21,690 \\
39,200\end{array}$ \\
\hline $\begin{array}{l}1905 \\
1910 \\
1915 \\
1920\end{array}$ & $\begin{array}{l}16,980 \\
19,910 \\
20,950 \\
21,280\end{array}$ & 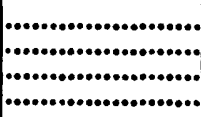 & $\begin{array}{l}25,470 \\
29,860 \\
31,420 \\
31,920\end{array}$ & $\begin{array}{l}27,500 \\
34,240 \\
37,950 \\
41,820\end{array}$ & $\begin{array}{l}46,510 \\
58,520 \\
65,420 \\
73,000\end{array}$ \\
\hline $\begin{array}{l}1921 \\
1922 \\
1923 \\
1924 \\
1925\end{array}$ & $\begin{array}{l}21,340 \\
21,400 \\
21,460 \\
21,520 \\
21,580\end{array}$ & $\begin{array}{l}794,000 \\
682,000 \\
224,000 \\
790,000\end{array}$ & $\begin{array}{l}32,010 \\
32,100 \\
32,190 \\
32,280 \\
32,370\end{array}$ & $\begin{array}{l}40,670 \\
39,840 \\
38,900 \\
38,410 \\
37,740\end{array}$ & $\begin{array}{l}70,670 \\
68,980 \\
67,070 \\
66,060 \\
64,690\end{array}$ \\
\hline $\begin{array}{l}1926 \\
1927 \\
1928 \\
1929 \\
1930\end{array}$ & $\begin{array}{l}21,630 \\
21,690 \\
21,750 \\
21,810 \\
21,870\end{array}$ & $\begin{array}{r}372,000 \\
1,060,000 \\
772,000 \\
368,000 \\
284,000\end{array}$ & $\begin{array}{l}32,440 \\
32,540 \\
32,620 \\
32,720 \\
32,800\end{array}$ & $\begin{array}{l}37,100 \\
37,100 \\
37,570 \\
38,530 \\
38,600\end{array}$ & $\begin{array}{l}63,380 \\
63,360 \\
64,260 \\
66,160 \\
66,260\end{array}$ \\
\hline $\begin{array}{l}1931 \\
1932 \\
1933 \\
1934 \\
1935\end{array}$ & $\begin{array}{l}21,930 \\
21,990 \\
22,040 \\
22,100 \\
22,100\end{array}$ & $\begin{array}{l}277,000 \\
750,000 \\
566,000 \\
358,600 \\
471,600\end{array}$ & $\begin{array}{l}32,900 \\
32,980 \\
33,060 \\
33,150 \\
33,150\end{array}$ & $\begin{array}{l}38,650 \\
38,420 \\
38,830 \\
39,250 \\
39,250\end{array}$ & $\begin{array}{l}66,340 \\
65,840 \\
66,640 \\
67,450 \\
67,450\end{array}$ \\
\hline $\begin{array}{l}1936 \\
1937 \\
1938 \\
1939 \\
1940\end{array}$ & $\begin{array}{l}22,100 \\
22,100 \\
22,100 \\
22,100 \\
22,100\end{array}$ & $\begin{array}{r}585,600 \\
362,400 \\
1,097,000 \\
431,400 \\
777,100\end{array}$ & $\begin{array}{l}33,150 \\
33,150 \\
33,150 \\
33,150 \\
33,150\end{array}$ & $\begin{array}{l}39,260 \\
39,260 \\
39,730 \\
40,180 \\
40,620\end{array}$ & $\begin{array}{l}67,470 \\
67,470 \\
68,410 \\
69,310 \\
70,190\end{array}$ \\
\hline $\begin{array}{l}1941 \\
1942 \\
1943 \\
1944 \\
1945 \\
1946\end{array}$ & $\begin{array}{l}22,100 \\
22,100 \\
22,100 \\
22,100 \\
22,100 \\
22,100\end{array}$ & $\begin{array}{r}727,800 \\
764,400 \\
1,005,000 \\
363,400 \\
683,000 \\
838,900\end{array}$ & $\begin{array}{l}33,150 \\
33,150 \\
33,150 \\
33,150 \\
33,150 \\
33,150\end{array}$ & $\begin{array}{l}40,620 \\
40,620 \\
40,620 \\
40,620 \\
40,620 \\
40,620\end{array}$ & $\begin{array}{l}70,190 \\
70,190 \\
70,190 \\
70,190 \\
70,190 \\
70,190\end{array}$ \\
\hline
\end{tabular}

- Irrigation is negligible above station on Crane Creek near Midvale, Idaho.

\section{SNAKE RIVER, MURPHY TO WEISER}

In addition to the subdivisions already discussed there are minor tracts of irrigated land supplied with water by small tributaries and by pumping directly from the Snake River.

Data on irrigated area and streamflow depletion for the entire Snake River basin above Weiser, Idaho, are given in the following table. The average streamflow depletion for the period 1921 through 1945 amounts to 24.2 percent of the yield of the basin above this point. In 1946 the estimated depletion had increased to 26.8 percent of the long-term yield. For the subdirision between Murphy and Weiser the average depletion over the base period amounted to 22.7 percent of the yield of this suldivision. In 1946 this had increased to 26.8 percent of the long-term yield. 
Irrigated area, annual runoff and estimated depletion for Snake River at Weiser, Idaho

\begin{tabular}{|c|c|c|c|}
\hline Year & $\begin{array}{l}\text { Irrigated area } \\
\text { (acres) }\end{array}$ & $\begin{array}{c}\text { Runoff } \\
\text { (acre-feet) }\end{array}$ & $\begin{array}{c}\text { Estimated } \\
\text { depletion } \\
\text { (acre-feet) }\end{array}$ \\
\hline $\begin{array}{l}1870 \\
1880 \\
1890 \\
1900\end{array}$ & $\begin{array}{r}10,287 \\
43,511 \\
229,383 \\
635,169\end{array}$ & 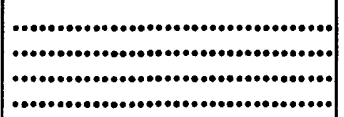 & $\begin{array}{r}20,650 \\
80,520 \\
373,480 \\
1,028,500\end{array}$ \\
\hline $\begin{array}{l}1905 \\
1910\end{array}$ & $\begin{array}{r}892,335 \\
1,312,724\end{array}$ & 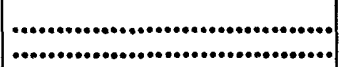 & $\begin{array}{l}1,462,130 \\
2,184,060\end{array}$ \\
\hline $\begin{array}{l}1911 \\
1912 \\
1913 \\
1914 \\
1915\end{array}$ & $\mid$ & $\begin{array}{l}15,900,000 \\
16,800,000 \\
16,200,000 \\
16,200,000 \\
10,400,000\end{array}$ & $\cdots \cdots \cdots \cdots \cdots \cdots \cdots \cdots \cdots \cdots \cdots \cdots \cdots \cdots \cdots \cdots$ \\
\hline $\begin{array}{l}1916 \\
1917 \\
1918 \\
1919 \\
1920\end{array}$ & $\mid$ & $\begin{array}{l}17,100,000 \\
18,000,000 \\
14,600,000 \\
12,200,000 \\
10,700,000\end{array}$ & 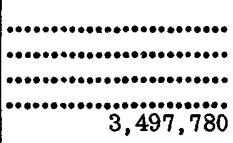 \\
\hline $\begin{array}{l}1921 \\
1922 \\
1923 \\
1924 \\
1925\end{array}$ & $\begin{array}{l}2,085,125 \\
2,090,104 \\
2,102,382 \\
2,054,345 \\
2,118,004\end{array}$ & $\begin{array}{r}18,300,000 \\
15,500,000 \\
12,800,000 \\
8,990,000 \\
14,300,000\end{array}$ & $\begin{array}{l}3,528,570 \\
3,568,390 \\
3,583,620 \\
3,521,410 \\
3,604,760\end{array}$ \\
\hline $\begin{array}{l}1926 \\
1927 \\
1928 \\
1929 \\
1930\end{array}$ & $\begin{array}{l}2,079,451 \\
2,148,542 \\
2,147,871 \\
2,155,761 \\
2,151,494\end{array}$ & $\begin{array}{r}10,200,000 \\
15,000,000 \\
16,400,000 \\
10,800,000 \\
9,500,000\end{array}$ & $\begin{array}{l}3,544,200 \\
3,665,060 \\
3,662,120 \\
3,676,200 \\
3,668,530\end{array}$ \\
\hline $\begin{array}{l}1931 \\
1932 \\
1933 \\
1934 \\
1935\end{array}$ & $\begin{array}{l}2,094,431 \\
2,160,994 \\
2,180,619 \\
2,080,736 \\
2,185,874\end{array}$ & $\begin{array}{r}7,880,000 \\
11,300,000 \\
9,840,000 \\
7,880,000 \\
8,136,000\end{array}$ & $\begin{array}{l}3,594,210 \\
3,687,690 \\
3,721,630 \\
3,569,520 \\
3,726,670\end{array}$ \\
\hline $\begin{array}{l}1936 \\
1937 \\
1938 \\
1939 \\
1940\end{array}$ & $\begin{array}{l}2,217,189 \\
2,259,635 \\
2,296,171 \\
2,320,250 \\
2,342,955\end{array}$ & $\begin{array}{r}11,320,000 \\
8,708,000 \\
14,790,000 \\
10,220,000 \\
10,520,000\end{array}$ & $\begin{array}{l}3,780,350 \\
3,785,600 \\
3,923,860 \\
3,968,060 \\
4,013,390\end{array}$ \\
\hline $\begin{array}{l}1941 \\
1942 \\
1943 \\
1944 \\
1945 \\
1946\end{array}$ & $\begin{array}{l}2,355,359 \\
2,382,386 \\
2,418,048 \\
2,437,315 \\
2,444,153 \\
2,455,892\end{array}$ & $\begin{array}{l}10,240,000 \\
12,110,000 \\
19,150,000 \\
10,970,000 \\
12,020,000 \\
15,410,000\end{array}$ & $\begin{array}{l}4,036,110 \\
4,086,010 \\
4,150,740 \\
4,186,860 \\
4,199,700 \\
4,220,570\end{array}$ \\
\hline
\end{tabular}

\section{BURNT RIVER}

The Burnt River rises in the Blue Mountains of eastern Oregon and drains about 1,100 square miles as it flows eastward to its confluence with the Snake River near Huntington. The uppor reaches of the basin are covered with timber and the lower reaches are characterized by rolling hills covered with sagebrush and grass. The bottom lands, which are devoted to agriculture, occur in three general areas: near Unity, Bridgeport, and Durkee. 
The irrigated area in the Burnt River basin is largely in small scattered tracts along the main stem; however some is along the tributary streams. (See the following table.) The main irrigation tracts are the Durkee Irrigation District and the Burnt River Irrigation District, both of which are below Unity Reservoir. The Burnt River also furnishes a small amount of water for lands within the Malheur River basin, via Eldorado Ditch.

Owing to water shortages that had shown up during years of low flow the Bureau of Reclamation, in 1937, constructed the Unity Reservoir just below the North and South Forks to supply supplemental water for lands already under irrigation. This reservoir has a usable capacity of 25,220 acre-feet.

The net consumptive use for lands within the basin has been estimated at 1.4 acre-feet per acre. The return flow from the lands above Unity Reservoir is assumed to be recaptiured by the main stem above the gaging station near Hereford. The return flow from lands below Unity Reservoir probably is recaptured by the Burnt River before it joins the Snake River, on which the next gaging station downstream is at Oxbow, Oreg., nearly 50 miles downstream. Based upon records for the period 1930 through 1946, the average depletion amounts to 19 percent of the yield above the gaging station near Hereford. 
Irrigated area, annual runoff, and estimated depletion for Burnt River basin

\begin{tabular}{|c|c|c|c|c|c|}
\hline \multirow[b]{2}{*}{ Year } & \multicolumn{3}{|c|}{ Burnt River near Hereford, Oreg. } & \multicolumn{2}{|c|}{ Burnt River at mouth } \\
\hline & $\begin{array}{l}\text { Irrigated area } \\
\text { (acres) }\end{array}$ & $\begin{array}{c}\text { Runoff } \\
\text { (acre-feet) }\end{array}$ & $\begin{array}{c}\text { Estimated } \\
\text { depletion } \\
\text { (acre-feet) }\end{array}$ & $\begin{array}{l}\text { Irrigated area } \\
\text { (acres) }\end{array}$ & $\begin{array}{l}\text { Estimated } \\
\text { depletion } \\
\text { (acre-feet) }\end{array}$ \\
\hline $\begin{array}{l}1870 \\
1880 \\
1890 \\
1900\end{array}$ & $\begin{array}{r}79 \\
501 \\
3,549 \\
5,256\end{array}$ & 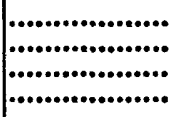 & $\begin{array}{r}110 \\
700 \\
4,970 \\
7,360\end{array}$ & $\begin{array}{r}1,050 \\
4,420 \\
14,200 \\
18,400\end{array}$ & $\begin{array}{r}1,470 \\
6,190 \\
19,880 \\
25,760\end{array}$ \\
\hline $\begin{array}{l}1905 \\
1910 \\
1915 \\
1920\end{array}$ & $\begin{array}{l}5,699 \\
6,499 \\
6,848 \\
7,706\end{array}$ & 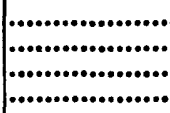 & $\begin{array}{r}7,980 \\
9,100 \\
9,590 \\
10,790\end{array}$ & $\begin{array}{l}20,000 \\
22,000 \\
24,000 \\
25,500\end{array}$ & $\begin{array}{l}28,000 \\
30,800 \\
33,600 \\
35,700\end{array}$ \\
\hline $\begin{array}{l}1921 \\
1922 \\
1923 \\
1924 \\
1925\end{array}$ & $\begin{array}{l}7,978 \\
8,270 \\
8,550 \\
8,639 \\
8,639\end{array}$ & 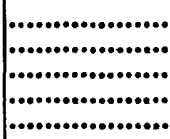 & $\begin{array}{l}11,170 \\
11,580 \\
11,970 \\
12,090 \\
12,090\end{array}$ & $\begin{array}{l}25,800 \\
26,300 \\
26,900 \\
27,100 \\
27,200\end{array}$ & $\begin{array}{l}36,120 \\
36,820 \\
37,660 \\
37,940 \\
38,080\end{array}$ \\
\hline $\begin{array}{l}1926 \\
1927 \\
1928 \\
1929 \\
1930\end{array}$ & $\begin{array}{l}8,639 \\
8,639 \\
8,639 \\
8,639 \\
8,639\end{array}$ & 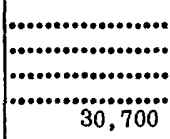 & $\begin{array}{l}12,090 \\
12,090 \\
12,090 \\
12,090 \\
12,090\end{array}$ & $\begin{array}{l}27,200 \\
27,400 \\
27,700 \\
27,900 \\
28,100\end{array}$ & $\begin{array}{l}38,080 \\
38,360 \\
38,780 \\
39,060 \\
39,340\end{array}$ \\
\hline $\begin{array}{l}1931 \\
1932 \\
1933 \\
1934 \\
1935\end{array}$ & $\begin{array}{l}8,639 \\
8,639 \\
8,659 \\
8,659 \\
8,659\end{array}$ & $\begin{array}{l}32,400 \\
66,900 \\
57,100 \\
22,460 \\
34,270\end{array}$ & $\begin{array}{l}12,090 \\
12,090 \\
12,120 \\
12,120 \\
12,120\end{array}$ & $\begin{array}{l}28,100 \\
28,300 \\
28,400 \\
28,400 \\
28,500\end{array}$ & $\begin{array}{l}39,340 \\
39,620 \\
39,760 \\
39,760 \\
39,900\end{array}$ \\
\hline $\begin{array}{l}1936 \\
1937 \\
1938 \\
1939 \\
1940\end{array}$ & $\begin{array}{l}8,659 \\
8,659 \\
8,659 \\
8,659 \\
8,659\end{array}$ & $\begin{array}{l}47,840 \\
30,770 \\
68,160 \\
39,980 \\
42,160\end{array}$ & $\begin{array}{l}12,120 \\
12,120 \\
12,120 \\
12,120 \\
12,120\end{array}$ & $\begin{array}{l}28,500 \\
28,500 \\
28,600 \\
28,600 \\
28,700\end{array}$ & $\begin{array}{l}39,900 \\
39,900 \\
40,040 \\
40,040 \\
40,180\end{array}$ \\
\hline $\begin{array}{l}1941 \\
1942 \\
1943 \\
1944 \\
1945 \\
1946\end{array}$ & $\begin{array}{l}8,659 \\
8,659 \\
8,659 \\
8,659 \\
8,659 \\
8,659\end{array}$ & $\begin{array}{r}52,090 \\
82,040 \\
107,100 \\
34,040 \\
46,450 \\
80,450\end{array}$ & $\begin{array}{l}12,120 \\
12,120 \\
12,120 \\
12,120 \\
12,120 \\
12,120\end{array}$ & $\begin{array}{l}29,000 \\
29,200 \\
29,400 \\
29,700 \\
29.700 \\
29,800\end{array}$ & $\begin{array}{l}40,600 \\
40,880 \\
41,160 \\
41,580 \\
41,580 \\
41,720\end{array}$ \\
\hline
\end{tabular}

POWDER RIVER

The Powder River heads in the Blue Mountains of eastern Oregon and flows eastward to a confluence with the Snake River near Robinette, Oreg., at the head of the Grand Canyon of the Snake River. The headwater area is generally mountainous, draining parts of the forested Elkhorn, Blue, and Wallowa Mountains. The lowland is roughly divided into a series of bowl-shaped valleys connected by successive short canyons. The principal lowland is around Baker and Haines; it contains about three-fourths of the irrigated land in all the drainage basin. 
The Powder River basin was settled comparatively early and some of its water rights date back to 1863 . Most of the irrigation works have been small and privately-financed ventures. Some lands are largely irrigated from subsurface sources. Hay, forage crops, and livestock are the principal agricultural products.

The Thief Valley Reservoir was constructed in 1932 by the $\mathrm{Bu}-$ reau of Reclamation to furnish supplemental water supply to the lands near Keating.

In the Big Creek subdivision there are approximately 3, 300 acres that are supplied by water from Catherine Creek, a tributary to the Grande Ronde River.

The areas shown in the following table are based upon adjudicated water rights, and are substantiated by a statement by $\mathrm{Mr}$. Maxwell, the watermaster for Baker County, that the areas are within 5 percent of the actual irrigated area. Ther $\epsilon$ are some late-season water shortages during years of low runoff and some lands received inadequate amounts of water in all years.

The net consumptive use for the lands in this basin has been estimated at 1.5 acre-feet per acre. For the purposes of this report, it is assumed that all return flow occurs above the gaging station on Powder River near Robinette.

Based upon the period, 1929 through 1946, the average depletion represents 33 percent of the yield of the basin above Robinette and it has remained essentially constant during this period. 
Irrigated area, annual runoff, and estimated depletion for Powder River near K: $: b i n e t t e$, Oreg.

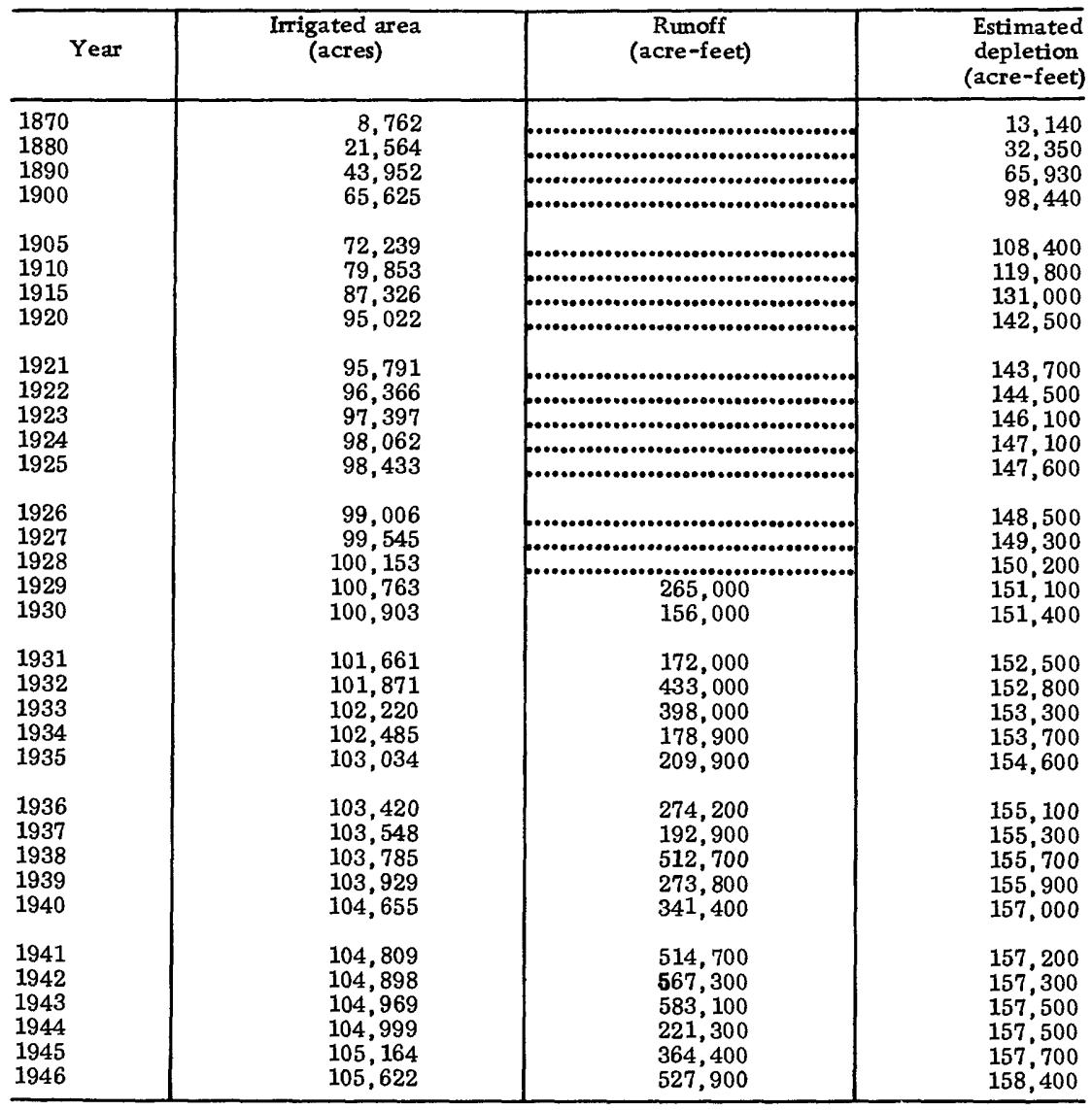

SNAKE RIVER, WEISER TO OXBOW

Other than in the Burnt and Powder River basins previously discussed, there are only minor amounts of irrigated land in this subdivision. The Snake River at Oxbow, Oreg., is below all the large, intensively irrigated areas and the return flow from all irrigated lands above this point has been recaptured by the mainstem river. The average streamflow depletion for the period, 1924 through 1946, represents 25 percent of the yield of the basin above the gaging station at Oxbow. The depletion, es shown in the following table, was increasing during the period of record and in 1946, amounted to 27.5 percent of the long-term yield. 
Irrigated area, annual runoff and estimated depletion for Snake River at Oxbow, Oreg.

\begin{tabular}{|c|c|c|c|}
\hline Year & $\begin{array}{c}\text { Irrigated area } \\
\text { (acres) }\end{array}$ & $\begin{array}{c}\text { Runoff } \\
\text { (acre-feet) }\end{array}$ & $\begin{array}{l}\text { Estimated } \\
\text { depletion } \\
\text { (acre-feet) }\end{array}$ \\
\hline $\begin{array}{l}1870 \\
1880 \\
1890 \\
1900\end{array}$ & $\begin{array}{r}20,099 \\
69,534 \\
287,815 \\
719,964\end{array}$ & 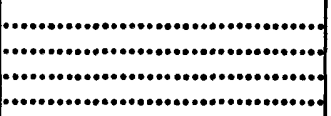 & $\begin{array}{r}35,260 \\
119,110 \\
459,690 \\
1,153,780\end{array}$ \\
\hline $\begin{array}{l}1905 \\
1910 \\
1915 \\
1920\end{array}$ & $\begin{array}{r}985,554 \\
1,415,997 \\
1,846,336 \\
2,167,260\end{array}$ & 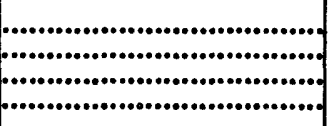 & $\begin{array}{l}1,599,900 \\
2,336,650 \\
3,119,010 \\
3,679,580\end{array}$ \\
\hline $\begin{array}{l}1921 \\
1922 \\
1923 \\
1924 \\
1925\end{array}$ & $\begin{array}{l}2,209,306 \\
2,215,390 \\
2,229,319 \\
2,182,177 \\
2,246,327\end{array}$ & $\begin{array}{r}9,420,000 \\
15,000,000\end{array}$ & $\begin{array}{l}3,712,020 \\
3,753,380 \\
3,771,080 \\
3,710,190 \\
3,794,210\end{array}$ \\
\hline $\begin{array}{l}1926 \\
1927 \\
1928 \\
1929 \\
1930\end{array}$ & $\begin{array}{l}2,208,357 \\
2,278,187 \\
2,278,424 \\
2,287,124 \\
2,283,197\end{array}$ & $\begin{array}{r}10,300,000 \\
15,500,000 \\
17,200,000 \\
11,400,000 \\
9,790,000\end{array}$ & $\begin{array}{l}3,744,560 \\
3,856,500 \\
3,854,880 \\
3,870,140 \\
3,863,050\end{array}$ \\
\hline $\begin{array}{l}1931 \\
1932 \\
1933 \\
1934 \\
1935\end{array}$ & $\begin{array}{l}2,226,892 \\
2,293,865 \\
2,313,939 \\
2,214,321 \\
2,320,108\end{array}$ & $\begin{array}{r}8,080,000 \\
11,900,000 \\
10,500,000 \\
8,197,000 \\
8,512,000\end{array}$ & $\begin{array}{l}3,789,830 \\
3,883,890 \\
3,918,470 \\
3,766,760 \\
3,924,950\end{array}$ \\
\hline $\begin{array}{l}1936 \\
1937 \\
1938 \\
1939 \\
1940\end{array}$ & $\begin{array}{l}2,351,809 \\
2,394,383 \\
2,431,256 \\
2,455,477 \\
2,479,010\end{array}$ & $\begin{array}{r}11,750,000 \\
9,058,000 \\
15,510,000 \\
10,670,000 \\
10,980,000\end{array}$ & $\begin{array}{l}3,979,130 \\
3,984,580 \\
4,123,380 \\
4,167,780 \\
4,214,350\end{array}$ \\
\hline $\begin{array}{l}1941 \\
1942 \\
1943 \\
1944 \\
1945 \\
1946\end{array}$ & $\begin{array}{l}2,491,898 \\
2,519,244 \\
2,555,207 \\
2,574,834 \\
2,581,877 \\
2,594,204\end{array}$ & $\begin{array}{l}11,010,000 \\
13,160,000 \\
19,750,000 \\
11,270,000 \\
12,420,000 \\
15,950,000\end{array}$ & $\begin{array}{l}4,237,730 \\
4,288,050 \\
4,353,310 \\
4,389,890 \\
4,402,980 \\
4,424,740\end{array}$ \\
\hline
\end{tabular}

PINE CREEK

Pine Creek drains about 280 square miles on the east slope of Wallowa Mountains in eastern Oregon, between the Powder and Imnaha drainage basins. The upper part of the basin is rugged and mountainous but the lower partincludes a fairly broad valley.

The irrigated lands (see the following table) have koen developed by private enterprise. There are numerous small lakes and reservoirs which are used for storage of irrigation water. Net consumptive use for this basin has been estimated at 1.4 acrefeet per acre. Return flow from these lands has been assumed to reach the Snake River downstream from the gaging station at Oxbow. 
Irrigated area for Pine Creek basin

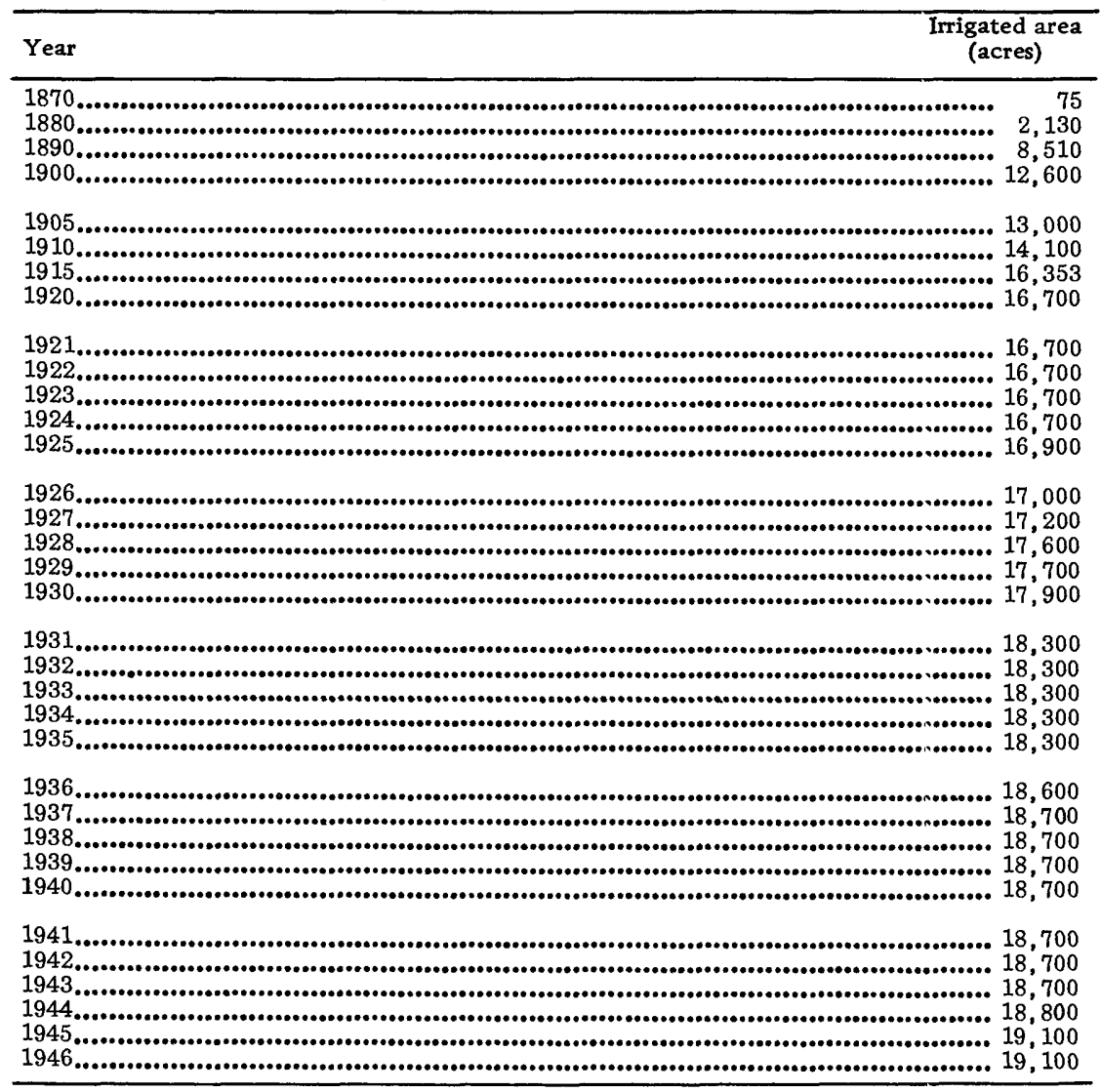

IMNAHA RIVER

The Imnaha River rises on the northern slope of the Wallowa Mountains in eastern Oregon and flows northeastward to its confluence with the Snake River, a short distance upstrear. from the mouth of the Salmon River. The drainage area is appriximately 900 square miles. The headwater area is rugged and mountainous, and to a large extent is forested. The lower portions of the basin are rolling hills covered with sagebrush and grass. There is very little agricultural land even in the narrow valley bottoms. Forage crops and stock are the chief products.

The irrigated lands are restricted to small tracts scattered throughout the basin. They have been developed by private enterprise and are not consolidated. The Wallowa Improvement District No. 1, in the Grande Ronde River basin, diverts water from Sheep Creek a tributary of the Imnaha River. The lands using this water have not been included in the following table but are included in the table on page 104 . 
It has been assumed, for the purpose of this report, that all acreage listed in the table below is above the gaging station on the Imnaha River at Imnaha and all return flow is recaptured by the stream above this point. This may not be strictly true, but in view of relatively small amounts involved it is believed that no appreciable error will result from this assumpticn. The net consumptive use for the acreages listed in the following table has been estimated at 1.2 acre-feet per acre. The average streamflow depletion over the period 1929 through 1946 amounts to 1.5 percent of the yield of the drainage basin and it has been practically constant during the period of record.

Irrigated area, annual runoff and estimated depletion for Imnana River at I mnaha, Oreg.

\begin{tabular}{|c|c|c|c|}
\hline Year & $\begin{array}{l}\text { Irrigated area } \\
\text { (acres) }\end{array}$ & $\begin{array}{c}\text { Runoff } \\
\text { (acre-feet) }\end{array}$ & $\begin{array}{l}\text { Estimated } \\
\text { depletion } \\
\text { (acre-feet) }\end{array}$ \\
\hline $\begin{array}{l}1880 \\
1890 \\
1900\end{array}$ & $\begin{array}{r}521 \\
1,480 \\
2,173\end{array}$ & 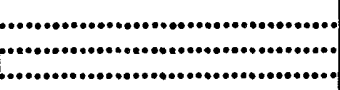 & $\begin{array}{r}620 \\
1,780 \\
2,610\end{array}$ \\
\hline $\begin{array}{l}1905 \\
1910 \\
1915 \\
1920\end{array}$ & $\begin{array}{l}2,370 \\
2,556 \\
2,727 \\
3,323\end{array}$ & 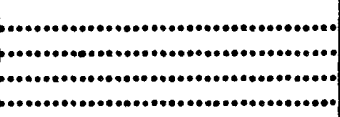 & $\begin{array}{l}2,840 \\
3,070 \\
3,270 \\
3,990\end{array}$ \\
\hline $\begin{array}{l}1921 \\
1922 \\
1923 \\
1924 \\
1925\end{array}$ & $\begin{array}{l}3,639 \\
3,647 \\
3,683 \\
3,707 \\
3,718\end{array}$ & 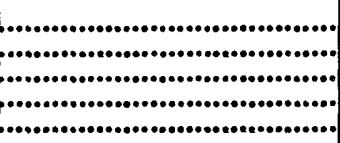 & $\begin{array}{l}4,370 \\
4,380 \\
4,420 \\
4,450 \\
4,460\end{array}$ \\
\hline $\begin{array}{l}1926 \\
1927 \\
1928 \\
1929 \\
1930\end{array}$ & $\begin{array}{l}3,718 \\
3,718 \\
3,722 \\
3,821 \\
3,837\end{array}$ & {$\left[\begin{array}{r} \\
282,000 \\
220,000\end{array}\right.$} & $\begin{array}{l}4,460 \\
4,460 \\
4,470 \\
4,580 \\
4,600\end{array}$ \\
\hline $\begin{array}{l}1931 \\
1932 \\
1933 \\
1934 \\
1935\end{array}$ & $\begin{array}{l}3,852 \\
3,852 \\
3,852 \\
3,913 \\
3,913\end{array}$ & $\begin{array}{l}179,000 \\
370,000 \\
360,000 \\
233,000 \\
266,800\end{array}$ & $\begin{array}{l}4,620 \\
4,620 \\
4,620 \\
4,700 \\
4,700\end{array}$ \\
\hline $\begin{array}{l}1936 \\
1937 \\
1938 \\
1939 \\
1940\end{array}$ & $\begin{array}{l}3,914 \\
3,914 \\
3,914 \\
3,914 \\
3,914\end{array}$ & $\begin{array}{l}226,600 \\
219,200 \\
546,100 \\
236,900 \\
290,600\end{array}$ & $\begin{array}{l}4,700 \\
4,700 \\
4,700 \\
4,700 \\
4,700\end{array}$ \\
\hline $\begin{array}{l}1941 \\
1942 \\
1943 \\
1944 \\
1945 \\
1946\end{array}$ & $\begin{array}{l}3,972 \\
3,972 \\
3,972 \\
3,972 \\
4,181 \\
4,200\end{array}$ & $\begin{array}{l}397,000 \\
496,600 \\
448,700 \\
241,600 \\
351,400 \\
394,600\end{array}$ & $\begin{array}{l}4,770 \\
4,770 \\
4,770 \\
4,770 \\
5,020 \\
5,040\end{array}$ \\
\hline
\end{tabular}


The Salmon River heads in the Sawtooth Mountains of central Idaho and flows generally north and west to join the Snake River near the lower end of its spectacular Grand Canyon. The drainage area of about 14,000 square miles, makes the Salmon River the largest of the Snake River tributaries from the standpoint of land drained. Rough, rugged terrain characterizes tris tributary basin. There is only a small amount of farm land and not much of that can be irrigated.

Irrigation was started in the Salmon River basin about 1860 in Lemhi County, near Salmon City. There was a steady increase in acreage under irrigation from 1880 to about 1920, after which time expansion has gradually tapered off. The irrication has mostly been by individuals, irrigation districts, or ccmpanies, backed by private capitol and has largely centered in four general areas; near Challis, in the Pahsimeroi Valley, in the Lemhi Valley and along the Little Salmon River near New Meadows. The Challis Irrigation District is a major organization in this area and it irrigates several thousand acres near Challis. Another large organization is the Keating Carey Land Co. which irrigates several thousand acres in the Lemhi Valley. Most of the other land in this tributary basin is irrigated from indivic'ually- or cooperatively-owned canals. The amount of irrigated area listed in the three tables following were computed primarily from data on water rights and reports by the State Engineer, Bureau of Reclamation, and Geological Survey.

Very little surface storage has been provided in this basin because the soil in the upper basin is, in general, highly permeable. The permeability factor has limited the amount of land that can be irrigated by a single canal.

A net consumptive use of 1.6 acre-feet per acre has been used for all lands in the Salmon River basin. It is assumed that neither the diversion nor the return flow bypasses any of the several gaging stations listed in the cited tables. The average streamflow depletion in this basin is relatively minor in comparison to its yield. For the area above the gaging station on Salmon River below Yankee Fork near Clayton, it amounts to only 1 fercent of long-term yield based on the period 1922 through 1946. Downstream at the gaging station near Challis it amounts to 1.5 percent of the long-term yield based upon the period 192? through 1946. The average depletion for the period 1921 through 1945 for the basinabove the gaging station at White Bird amounts to 2 percent of the yield of the basin above this point. 
Irrigated area, annual runoff and estimated depletion for upper Salmon Piver basin

\begin{tabular}{|c|c|c|c|c|c|}
\hline \multirow{2}{*}{ Year } & \multirow{2}{*}{$\begin{array}{c}\begin{array}{c}\text { Salmon River } \\
\text { near } \\
\text { Obsidian, Idaho }\end{array} \\
\begin{array}{c}\text { Irrigated area } \\
\text { (acres) }\end{array}\end{array}$} & \multirow{2}{*}{$\begin{array}{c}\text { Valley Creek } \\
\text { at } \\
\text { Stanley, Idaho } \\
\begin{array}{c}\text { Irrigated area } \\
\text { (acres) }\end{array}\end{array}$} & \multicolumn{3}{|c|}{$\begin{array}{c}\text { Salmon River below Yankee Fork } \\
\text { near Clayton, Id:aho }\end{array}$} \\
\hline & & & $\begin{array}{l}\text { Irrigated area } \\
\text { (acres) }\end{array}$ & $\underset{\text { (acre-feet) }}{\text { Runoff }}$ & $\begin{array}{l}\text { Estimated } \\
\text { depletion } \\
\text { (acre-feet) }\end{array}$ \\
\hline $\begin{array}{l}1905 \\
1910 \\
1915 \\
1920\end{array}$ & $\begin{array}{r}30 \\
510 \\
\\
\end{array}$ & $\begin{array}{r}90 \\
240 \\
340 \\
440\end{array}$ & $\begin{array}{r}90 \\
440 \\
940 \\
2,500\end{array}$ & 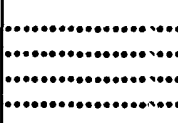 & $\begin{array}{r}140 \\
700 \\
1,500 \\
4,000\end{array}$ \\
\hline $\begin{array}{l}1921 \\
1922 \\
1923 \\
1924 \\
1925\end{array}$ & $\begin{array}{l}640 \\
770 \\
860 \\
960 \\
960\end{array}$ & $\begin{array}{l}450 \\
470 \\
480 \\
490 \\
490\end{array}$ & $\begin{array}{l}2,790 \\
2,990 \\
3,140 \\
3,280 \\
3,320\end{array}$ & 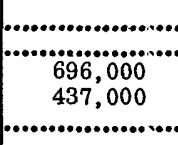 & $\begin{array}{l}4,460 \\
4,780 \\
5,020 \\
5,250 \\
5,310\end{array}$ \\
\hline $\begin{array}{l}1926 \\
1927 \\
1928 \\
1929 \\
1930\end{array}$ & $\begin{array}{l}960 \\
960 \\
960 \\
960 \\
960\end{array}$ & $\begin{array}{l}500 \\
500 \\
500 \\
500 \\
500\end{array}$ & $\begin{array}{l}3,360 \\
3,360 \\
3,360 \\
3,360 \\
3,360\end{array}$ & $\begin{array}{l}444,000 \\
848,000 \\
852,000 \\
498,000 \\
566,000\end{array}$ & $\begin{array}{l}5,380 \\
5,380 \\
5,380 \\
5,380 \\
5,380\end{array}$ \\
\hline $\begin{array}{l}1931 \\
1932 \\
1933 \\
1934 \\
1935\end{array}$ & $\begin{array}{r}960 \\
960 \\
960 \\
1,100 \\
1,200\end{array}$ & $\begin{array}{l}500 \\
500 \\
500 \\
500 \\
500\end{array}$ & $\begin{array}{l}3,360 \\
3,360 \\
3,360 \\
3,500 \\
3,700\end{array}$ & $\begin{array}{l}415,000 \\
675,000 \\
624,000 \\
532,300 \\
576,500\end{array}$ & $\begin{array}{l}5,380 \\
5,380 \\
5,380 \\
5,600 \\
5,920\end{array}$ \\
\hline $\begin{array}{l}1936 \\
1937 \\
1938 \\
1939 \\
1940\end{array}$ & $\begin{array}{l}1,300 \\
1,400 \\
1,550 \\
1,600 \\
1,600\end{array}$ & $\begin{array}{l}500 \\
500 \\
500 \\
500 \\
510\end{array}$ & $\begin{array}{l}3,900 \\
4,200 \\
4,550 \\
4,800 \\
5,110\end{array}$ & $\begin{array}{l}699,900 \\
428,800 \\
818,200 \\
493,900 \\
584,700\end{array}$ & $\begin{array}{l}6,240 \\
6,720 \\
7,280 \\
7,680 \\
8,180\end{array}$ \\
\hline $\begin{array}{l}1941 \\
1942 \\
1943 \\
1944 \\
1945 \\
1946\end{array}$ & $\begin{array}{l}1,700 \\
1,700 \\
1,700 \\
1,700 \\
1,700 \\
1,700\end{array}$ & $\begin{array}{l}520 \\
540 \\
550 \\
560 \\
570 \\
590\end{array}$ & $\begin{array}{l}5,420 \\
5,740 \\
5,950 \\
5,960 \\
5,970 \\
5,990\end{array}$ & $\begin{array}{r}553,300 \\
713,700 \\
1,139,000 \\
610,600 \\
571,300 \\
753,900\end{array}$ & $\begin{array}{l}8,670 \\
9,180 \\
9,520 \\
9,540 \\
9,550 \\
9,580\end{array}$ \\
\hline
\end{tabular}


SNAKE RIVER BASIN

Irrigated area, annual runoff and estimated depletion for central Salmon River basin

\begin{tabular}{|c|c|c|c|c|c|}
\hline \multirow{2}{*}{ Year } & \multicolumn{3}{|c|}{ Salmon River near Challis, Idaho } & \multirow{2}{*}{$\begin{array}{c}\begin{array}{c}\text { Pahsimeroi River } \\
\text { near } \\
\text { May, Idaho }\end{array} \\
\begin{array}{c}\text { Irrigated area } \\
\text { (acres) }\end{array}\end{array}$} & \multirow{2}{*}{$\begin{array}{c}\begin{array}{c}\text { North Fork Salmon } \\
\text { River at North } \\
\text { Fork, Idaho }\end{array} \\
\begin{array}{c}\text { Irigated area } \\
\text { (acres) }\end{array}\end{array}$} \\
\hline & $\begin{array}{l}\text { Irrigated area } \\
\text { (acres) }\end{array}$ & $\begin{array}{c}\text { Annual } \\
\text { runoff } \\
\text { (acre-feet) }\end{array}$ & $\begin{array}{l}\text { Estimated } \\
\text { depletion } \\
\text { (acre-feet) }\end{array}$ & & \\
\hline $\begin{array}{l}1880 \\
1890 \\
1900\end{array}$ & $\begin{array}{r}200 \\
3,270 \\
3,500\end{array}$ & & $\begin{array}{r}300 \\
5,230 \\
5,600\end{array}$ & $\begin{array}{r}4,290 \\
11,470\end{array}$ & 290 \\
\hline $\begin{array}{l}1905 \\
1910 \\
1915 \\
1920\end{array}$ & $\begin{array}{l}3,590 \\
4,040 \\
4,930 \\
6,800\end{array}$ & ................... & $\begin{array}{r}5,740 \\
6,460 \\
7,890 \\
10,900\end{array}$ & $\begin{array}{l}12,490 \\
12,490 \\
12,490 \\
12,490\end{array}$ & $\begin{array}{l}637 \\
770 \\
820 \\
820\end{array}$ \\
\hline $\begin{array}{l}1921 \\
1922 \\
1923 \\
1924 \\
1925\end{array}$ & $\begin{array}{l}7,090 \\
7,290 \\
7,440 \\
7,580 \\
7,620\end{array}$ & & $\begin{array}{l}11,300 \\
11,700 \\
11,900 \\
12,100 \\
12,200\end{array}$ & $\begin{array}{l}12,490 \\
12,490 \\
12,490 \\
12,490 \\
12,490\end{array}$ & $\begin{array}{l}820 \\
820 \\
820 \\
820 \\
820\end{array}$ \\
\hline $\begin{array}{l}1926 \\
1927 \\
1928 \\
1929 \\
1930\end{array}$ & $\begin{array}{l}7,660 \\
7,660 \\
7,660 \\
7,660 \\
7,660\end{array}$ & $\begin{array}{l}768,000 \\
878,000\end{array}$ & $\begin{array}{l}12,300 \\
12,300 \\
12,300 \\
12,300 \\
12,300\end{array}$ & $\begin{array}{l}12,490 \\
12,490 \\
12,490 \\
12,490 \\
12,490\end{array}$ & $\begin{array}{l}820 \\
820 \\
820 \\
820 \\
820\end{array}$ \\
\hline $\begin{array}{l}1931 \\
1932 \\
1933 \\
1934 \\
1935\end{array}$ & $\begin{array}{l}7,660 \\
7,660 \\
7,660 \\
7,800 \\
8,000\end{array}$ & $\begin{array}{r}619,000 \\
1,010,000 \\
910,000 \\
735,400 \\
854,800\end{array}$ & $\begin{array}{l}12,300 \\
12,300 \\
12,300 \\
12,500 \\
12,800\end{array}$ & $\begin{array}{l}12,490 \\
12,490 \\
12,490 \\
12,490 \\
12,490\end{array}$ & $\begin{array}{l}820 \\
820 \\
820 \\
820 \\
820\end{array}$ \\
\hline $\begin{array}{l}1936 \\
1937 \\
1938 \\
1939 \\
1940\end{array}$ & $\begin{array}{l}8,200 \\
8,500 \\
8,850 \\
9,100 \\
9,410\end{array}$ & $\begin{array}{r}1,015,000 \\
636,900 \\
1,218,000 \\
738,800 \\
854,800\end{array}$ & $\begin{array}{l}13,100 \\
13,600 \\
14,200 \\
14,600 \\
15,100\end{array}$ & $\begin{array}{l}12,490 \\
12,490 \\
12,490 \\
12,490 \\
12,490\end{array}$ & $\begin{array}{l}820 \\
830 \\
830 \\
830 \\
840\end{array}$ \\
\hline $\begin{array}{l}1941 \\
1942 \\
1943 \\
1944 \\
1945 \\
1946\end{array}$ & $\begin{array}{r}9,720 \\
10,040 \\
10,250 \\
10,260 \\
10,270 \\
10,290\end{array}$ & $\begin{array}{r}835,200 \\
1,100,000 \\
1,667,000 \\
967,800 \\
846,200 \\
1,092,000\end{array}$ & $\begin{array}{l}15,600 \\
16,100 \\
16,400 \\
16,400 \\
16,400 \\
16,500\end{array}$ & $\begin{array}{l}12,490 \\
12,490 \\
12,490 \\
12,490 \\
12,490 \\
12,490\end{array}$ & $\begin{array}{l}840 \\
840 \\
840 \\
840 \\
840 \\
840\end{array}$ \\
\hline
\end{tabular}


Irrigated area, annual runoff, and estimated depletion for Salmon River tasin

\begin{tabular}{|c|c|c|c|c|c|}
\hline \multirow{2}{*}{ Year } & \multirow{2}{*}{$\begin{array}{c}\begin{array}{c}\text { Salmon River } \\
\text { near } \\
\text { French Creek, Idaho }\end{array} \\
\begin{array}{c}\text { Irigated area } \\
\text { (acres) }\end{array}\end{array}$} & \multirow{2}{*}{$\begin{array}{c}\begin{array}{c}\text { Little Salmon } \\
\text { River basin }\end{array} \\
\begin{array}{c}\text { Irrigated area } \\
\text { (acres) }\end{array}\end{array}$} & \multicolumn{3}{|c|}{ Salmon River at White Bird, Idaho } \\
\hline & & & $\begin{array}{l}\text { Irrigated area } \\
\text { (acres) }\end{array}$ & $\begin{array}{c}\text { Runoff } \\
\text { (acre-feet) }\end{array}$ & $\begin{array}{c}\text { Estimated } \\
\text { depletion } \\
\text { (acre-feet) }\end{array}$ \\
\hline $\begin{array}{l}1870 \\
1880 \\
1890 \\
1900\end{array}$ & $\begin{array}{r}530 \\
5,940 \\
24,810 \\
41,140\end{array}$ & $\begin{array}{r}35 \\
990 \\
3,670\end{array}$ & $\begin{array}{r}530 \\
5,970 \\
26,000 \\
45,510\end{array}$ & ( & $\begin{array}{r}850 \\
9,550 \\
41,600 \\
72,800\end{array}$ \\
\hline $\begin{array}{l}1905 \\
1910\end{array}$ & $\begin{array}{l}48,420 \\
54,600\end{array}$ & $\begin{array}{l}4,970 \\
6,550\end{array}$ & $\begin{array}{l}54,750 \\
63,240\end{array}$ & ( & $\begin{array}{r}87,600 \\
101,200\end{array}$ \\
\hline $\begin{array}{l}1911 \\
1912 \\
1913 \\
1914 \\
1915\end{array}$ & 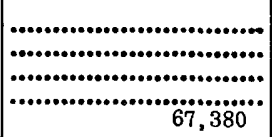 & ( & 78,060 & $\begin{array}{l}9,480,000 \\
9,260,000 \\
9,570,000 \\
7,330,000 \\
5,690,000\end{array}$ & ( \\
\hline $\begin{array}{l}1916 \\
1917\end{array}$ & 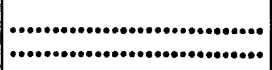 & & & $\begin{array}{r}10,600,000 \\
9,320,000\end{array}$ & \\
\hline$\ddot{1920}$ & $\cdots \cdots \cdots \cdots \cdots \cdots$ & 9,900 & $\ddot{92,960}$ & $\cdots, 6,970,000$ & $\cdots \cdots \cdots \cdots$ \\
\hline $\begin{array}{l}1921 \\
1922 \\
1923 \\
1924 \\
1925\end{array}$ & $\begin{array}{l}81,700 \\
82,630 \\
83,330 \\
84,080 \\
84,634\end{array}$ & $\begin{array}{l}10,320 \\
10,740 \\
11,160 \\
11,580 \\
12,000\end{array}$ & $\begin{array}{l}94,640 \\
95,990 \\
97,110 \\
98,280 \\
99,250\end{array}$ & $\begin{array}{r}10,800,000 \\
8,040,000 \\
7,130,000 \\
5,030,000 \\
8,190,000\end{array}$ & $\begin{array}{l}151,400 \\
153,600 \\
155,400 \\
157,200 \\
158,800\end{array}$ \\
\hline $\begin{array}{l}1926 \\
1927 \\
1928 \\
1929 \\
1930\end{array}$ & $\begin{array}{l}84,977 \\
85,301 \\
85,410 \\
85,570 \\
85,740\end{array}$ & $\begin{array}{l}12,280 \\
12,560 \\
12,840 \\
13,120 \\
13,400\end{array}$ & $\begin{array}{r}99,880 \\
100,480 \\
100,880 \\
101,330 \\
101,820\end{array}$ & $\begin{array}{l}4,960,000 \\
9,110,000 \\
9,330,000 \\
5,890,000 \\
6,190,000\end{array}$ & $\begin{array}{l}159,800 \\
160,800 \\
161,400 \\
162,100 \\
162,900\end{array}$ \\
\hline $\begin{array}{l}1931 \\
1932 \\
1933 \\
1934 \\
1935\end{array}$ & $\begin{array}{l}85,790 \\
85,920 \\
85,930 \\
86,090 \\
86,300\end{array}$ & $\begin{array}{l}13,420 \\
13,440 \\
13,460 \\
13,480 \\
13,500\end{array}$ & $\begin{array}{l}101,930 \\
102,120 \\
102,200 \\
102,440 \\
102,730\end{array}$ & $\begin{array}{l}4,200,000 \\
7,940,000 \\
7,410,000 \\
5,536,000 \\
5,827,000\end{array}$ & $\begin{array}{l}163,100 \\
163,400 \\
163,500 \\
163,900 \\
164,400\end{array}$ \\
\hline $\begin{array}{l}1936 \\
1937 \\
1938 \\
1939 \\
1940\end{array}$ & $\begin{array}{l}86,520 \\
86,940 \\
87,300 \\
87,570 \\
87,910\end{array}$ & $\begin{array}{l}13,510 \\
13,510 \\
13,520 \\
13,530 \\
13,530\end{array}$ & $\begin{array}{l}103,000 \\
103,480 \\
103,900 \\
104,200 \\
104,560\end{array}$ & $\begin{array}{l}7,106,000 \\
4,687,000 \\
8,907,000 \\
5,857,000 \\
6,460,000\end{array}$ & $\begin{array}{l}164,800 \\
165,600 \\
166,200 \\
166,700 \\
167,300\end{array}$ \\
\hline $\begin{array}{l}1941 \\
1942 \\
1943 \\
1944 \\
1945 \\
1946\end{array}$ & $\begin{array}{l}88,240 \\
88,580 \\
88,810 \\
88,850 \\
88,880 \\
88,920\end{array}$ & $\begin{array}{l}13,530 \\
13,540 \\
13,540 \\
13,550 \\
13,550 \\
13,600\end{array}$ & $\begin{array}{l}104,920 \\
105,270 \\
105,520 \\
105,580 \\
105,620 \\
105,720\end{array}$ & $\begin{array}{r}6,444,000 \\
8,786,000 \\
11,480,000 \\
6,107,000 \\
6,576,000 \\
7,596,000\end{array}$ & $\begin{array}{l}167,900 \\
168,400 \\
168,800 \\
168,900 \\
169,000 \\
169,200\end{array}$ \\
\hline
\end{tabular}

GRANDE RONDE RIVER

The Grande Ronde River drains 4,070 square miles, of which most is in northeastern Oregon and only a very small part, near the mouth of the stream, is in southeastern Washington. The stream rises on the eastern slope of the Blue Mountains and flows generally northeastward to its confluence with the Snake River. The upper and lower parts of the basin are mountainous; the agricultural land lies near the center of the basin in the valleys of the Grande Ronde and the Wallowa Rivers. 
These two valley areas were irrigated by private enterprise. Some of the adjudicated rights date back into the decac'e of 186070. By 1900 about half of the lands currently irrigated had been developed. In the valley of the Grande Ronde the irrigated land is between Union, Cove, La Grande and Elgin. Poor drainage and a consequent high water table are local problems in this basin. In this report the irrigated acreage (table p. 104) was derived after a field reconnaissance and a study of the water rights by the county agent, the watermaster for Union County, and representatives of the Survey. One of the difficulties in reaching an accurate determination of irrigated area in this valley has been the combination of relatively high precipitation and a high water table. Under these circumstances the amount of land actually irrigated in any year depends to a large extent upon variations in precipitation. Nevertheless water is used consumptively regardless of its source, and so all lands with an active water right have been classified as irrigated. Water is diverted from Catherine Creek, a tributary of the Grande Ronde, for use ir the Powder River basin. The land using this water has been included in the table on page 95 .

Irrigation in the Wallowa River valley has paralleled, to a large degree, that on the Grande Ronde River valley except that there has been very little increase in acreage of land irrigated during the past 30 years. The irrigated land, some 54,000 acres, as shown in the following table, lies between Wallowa Lake and Wallowa.

Since 1906 the Wallowa Improvement District No. 1 has received a portion of its water supply by diversion from Sheep Creek, a tributary of the Imnaha River. The lands using this water have been included in the following table. About 41,000 acre-feet storage capacity is available in Wallowa Lake.

In addition to these two valleys, a few hundred acres of land are irrigated with water from Joseph Creek. These have been included in the following table for purposes of estimating depletion for the subdivision. A netconsumptive use of 1.2 acre-feet per acre was used for the irrigated land in the entire subdivision. For purposes of this report, it was assumed that all diversion and return flow occurs above the gaging station on Grande Ronde River at Rondowa. The average depletion for period 1927 through 1946 amounts to 7.4 percent of the yield of the basin above the gaging station at Rondowa and has changed very slightly over the period of record. 
Irrigated area, anmual runoff, and estimated depletion for Grande Ronde River basin

\begin{tabular}{|c|c|c|c|c|}
\hline \multirow{2}{*}{ Year } & \multirow{2}{*}{$\begin{array}{c}\text { Wallowa River } \\
\text { basin }\end{array}$} & \multicolumn{3}{|c|}{ Grande Ronde River at Rondowa, Ore?. } \\
\hline & & $\begin{array}{l}\text { Irrigated area } \\
\quad \text { (acres) }\end{array}$ & $\begin{array}{c}\text { Runoff } \\
\text { (acre-feet) }\end{array}$ & $\begin{array}{c}\text { Estimated } \\
\text { depletion } \\
\text { (acre-feet) }\end{array}$ \\
\hline $\begin{array}{l}1870 \\
1880 \\
1890 \\
1900\end{array}$ & $\begin{array}{c}1,041 \\
10,402 \\
30,529\end{array}$ & $\begin{array}{r}7,260 \\
14,988 \\
28,495 \\
51,316\end{array}$ & | & $\begin{array}{r}8,710 \\
18,000 \\
34,200 \\
61,600\end{array}$ \\
\hline $\begin{array}{l}1905 \\
1910 \\
1915 \\
1920\end{array}$ & $\begin{array}{l}40,415 \\
42,831 \\
44,211 \\
51,173\end{array}$ & $\begin{array}{l}65,481 \\
70,855 \\
77,066 \\
84,701\end{array}$ & | & $\begin{array}{r}78,600 \\
85,000 \\
92,500 \\
101,600\end{array}$ \\
\hline $\begin{array}{l}1921 \\
1922 \\
1923 \\
1924 \\
1925\end{array}$ & $\begin{array}{l}\mathbf{5 1}, 376 \\
\mathbf{5 1}, 709 \\
\mathbf{5 1}, 830 \\
\mathbf{5 1}, 987 \\
\mathbf{5 2}, 207\end{array}$ & $\begin{array}{l}85,160 \\
85,720 \\
86,136 \\
86,517 \\
86,993\end{array}$ & 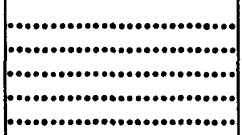 & $\begin{array}{l}102,200 \\
102,900 \\
103,400 \\
103,800 \\
104,400\end{array}$ \\
\hline $\begin{array}{l}1926 \\
1927 \\
1928 \\
1929 \\
1930\end{array}$ & $\begin{array}{l}\mathbf{5 2}, \mathbf{5 7 0} \\
\mathbf{5 2}, 698 \\
\mathbf{5 2}, 871 \\
\mathbf{5 3}, 035 \\
\mathbf{5 3}, 389\end{array}$ & $\begin{array}{l}87,682 \\
88,049 \\
88,373 \\
88,682 \\
89,213\end{array}$ & $\begin{array}{r}1,650,000 \\
2,050,000 \\
1,130,000 \\
977,000\end{array}$ & $\begin{array}{l}105,200 \\
105,700 \\
106,000 \\
106,400 \\
107,100\end{array}$ \\
\hline $\begin{array}{l}1931 \\
1932 \\
1933 \\
1934 \\
1935\end{array}$ & $\begin{array}{l}53,480 \\
53,578 \\
53,619 \\
53,686 \\
53,935\end{array}$ & $\begin{array}{l}89,347 \\
89,501 \\
89,697 \\
89,816 \\
90,168\end{array}$ & $\begin{array}{r}876,000 \\
1,850,000 \\
1,640,000 \\
1,069,000 \\
1,092,000\end{array}$ & $\begin{array}{l}107,200 \\
107,400 \\
107,600 \\
107,800 \\
108,200\end{array}$ \\
\hline $\begin{array}{l}1936 \\
1937 \\
1938 \\
1939 \\
1940\end{array}$ & $\begin{array}{l}54,011 \\
54,203 \\
54,318 \\
54,378 \\
54,501\end{array}$ & $\begin{array}{l}90,950 \\
91,762 \\
92,596 \\
92,936 \\
93,495\end{array}$ & $\begin{array}{r}1,356,000 \\
999,200 \\
1,418,000 \\
1,186,000 \\
1,121,000\end{array}$ & $\begin{array}{l}109,100 \\
110,100 \\
111,100 \\
111,500 \\
112,200\end{array}$ \\
\hline $\begin{array}{l}1941 \\
1942 \\
1943 \\
1944 \\
1945 \\
1946\end{array}$ & $\begin{array}{l}54,606 \\
54,669 \\
54,727 \\
54,769 \\
54,769 \\
54,846\end{array}$ & $\begin{array}{l}93,642 \\
93,705 \\
93,763 \\
93,805 \\
94,105 \\
94,449\end{array}$ & $\begin{array}{r}1,214,000 \\
1,682,000 \\
2,138,000 \\
925,200 \\
1,323,000 \\
1,800,000\end{array}$ & $\begin{array}{l}112,400 \\
112,400 \\
112,500 \\
112,600 \\
112,900 \\
113,300\end{array}$ \\
\hline
\end{tabular}

\section{CLEARWATER RIVER}

The Clearwater River rises on the western slope of the Bitterroot Mountains and flows generally west to its confluence with the Snake River at Lewiston, Idaho. Most of the basin is covered with timber and only a very small part of the agricultural land is irrigated. (See the following table.)

There are approximately 3,700 acres of irrigated land within this basin, of which 2,900 acres are within the Lewiston Orchard Irrigation District. The remainder is scattered, principally between Kamiah, Idaho and Clarkston, Wash.

The Lewiston Orchard Irrigation District is located on the bench just east of Lewiston, Idaho. This project was started ir 1906 by private capital. However, the Bureau of Reclamation undertook 
to rehabilitate the system for distribution of water in 1947. The project area is highly subdivided, with the average individual tract being 5 acres. Water supply, which is received from several creeks just east of the project, is delivered in pressure conduits.

The net consumptive use was estimated at 1.5 acre-feet per acre, based upon computations by Lowry-Johnson method. Return flow from these lands is captured by the Snake River above the gaging station near Clarkston.

Irrigated area, and estimated depletion for Clearwater River basin

\begin{tabular}{|c|c|c|c|}
\hline \multirow{2}{*}{ Year } & \multirow{2}{*}{$\begin{array}{c}\begin{array}{c}\text { Clearwater River } \\
\text { at Kamiah, Idaho }\end{array} \\
\begin{array}{c}\text { Irrigated area } \\
\text { (acres) }\end{array}\end{array}$} & \multicolumn{2}{|c|}{ Clearwater River at nouth } \\
\hline & & $\begin{array}{l}\text { Irrigated area } \\
\text { (acres) }\end{array}$ & $\begin{array}{c}\text { Estimated } \\
\text { depletion } \\
\text { (acre-feet) }\end{array}$ \\
\hline $\begin{array}{l}1890 \\
1900\end{array}$ & • & $\begin{array}{r}550 \\
1,750\end{array}$ & $\begin{array}{r}820 \\
2,620\end{array}$ \\
\hline $\begin{array}{l}1905 \\
1910 \\
1915 \\
1920\end{array}$ & $\begin{array}{r}19 \\
36 \\
56\end{array}$ & $\begin{array}{l}1,860 \\
3,960 \\
3,860 \\
3,860\end{array}$ & $\begin{array}{l}2,790 \\
5,940 \\
5,790 \\
5,790\end{array}$ \\
\hline $\begin{array}{l}1921 \\
1922 \\
1923 \\
1924 \\
1925\end{array}$ & $\begin{array}{r}60 \\
75 \\
90 \\
100 \\
120\end{array}$ & $\begin{array}{l}3,870 \\
3,810 \\
3,760 \\
3,690 \\
3,630\end{array}$ & $\begin{array}{l}5,800 \\
5,720 \\
5,640 \\
5,540 \\
5,440\end{array}$ \\
\hline $\begin{array}{l}1926 \\
1927 \\
1928 \\
1929 \\
1930\end{array}$ & $\begin{array}{l}140 \\
150 \\
160 \\
180 \\
200\end{array}$ & $\begin{array}{l}3,570 \\
3,480 \\
3,430 \\
3,410 \\
3,350\end{array}$ & $\begin{array}{l}5,360 \\
5,220 \\
5,140 \\
5,120 \\
5,020\end{array}$ \\
\hline $\begin{array}{l}1931 \\
1932 \\
1933 \\
1934 \\
1935\end{array}$ & $\begin{array}{l}210 \\
220 \\
240 \\
260 \\
280\end{array}$ & $\begin{array}{l}3,370 \\
3,380 \\
3,400 \\
3,410 \\
3,450\end{array}$ & $\begin{array}{l}5,060 \\
5,070 \\
5,100 \\
5,120 \\
5,180\end{array}$ \\
\hline $\begin{array}{l}1936 \\
1937 \\
1938 \\
1939 \\
1940\end{array}$ & $\begin{array}{l}300 \\
310 \\
330 \\
340 \\
360\end{array}$ & $\begin{array}{l}3,490 \\
3,500 \\
3,550 \\
3,560 \\
3,460\end{array}$ & $\begin{array}{l}5,180 \\
5,250 \\
5,320 \\
5,340 \\
5,190\end{array}$ \\
\hline $\begin{array}{l}1941 \\
1942 \\
1943 \\
1944 \\
1945 \\
1946\end{array}$ & $\begin{array}{l}370 \\
380 \\
390 \\
400 \\
410 \\
420\end{array}$ & $\begin{array}{l}3,470 \\
3,480 \\
3,500 \\
3,510 \\
3,520 \\
3,630\end{array}$ & $\begin{array}{l}5,200 \\
5,220 \\
5,250 \\
5,260 \\
5,280 \\
5,440\end{array}$ \\
\hline
\end{tabular}




\section{SNAKE RIVER, OXBOW TO CLARKSTON}

There are only minor areas of irrigation between the gaging stations on the Snake River at Oxbow and near Clarkston, other than in the subdivision already discussed. The station near Clarkston is the furthest downstream station on the main stem of the Snake River for which discharge records are available and as such, affords an opportunity to evaluate the aggregate streamflow depletion that can be directly attributed to the irrigation in the Snake River basin. The average depletion during the period 1929 through 1946, amounts to 12.7 percent of the yield of the basin above the gaging station near Clarkston. During this period, the irrigated area has been increasing, and the depletion for 1946 represents 13.7 percent of the long-term yield, 19 percent of the yield during 1931 , or 8.8 percent of the yield during 1943. These values indicate the percentage of the yield of the basin, during an average, a low, and a high water year respectively, which is used consumptively by the 1946 stage of irrigation development in the Snake River basin. The irrigated area and estimated depletion above the gaging station Snake River near Clarkston is shown in the following table, and for the Columbia Fiver below the Snake River in the table on page 108. 
Irrigated area, annual runoff, and estimated depletion for Snake River near Clarkston, Wash.

\begin{tabular}{|c|c|c|c|}
\hline Year & $\begin{array}{l}\text { Irrigated area } \\
\text { (acres) }\end{array}$ & $\begin{array}{c}\text { Runoff } \\
\text { (acre-feet) }\end{array}$ & $\begin{array}{c}\text { Estimated } \\
\text { depletion } \\
\text { (acre-feet) }\end{array}$ \\
\hline $\begin{array}{l}1870 \\
1880 \\
1890 \\
1900\end{array}$ & $\begin{array}{r}27,964 \\
93,143 \\
352,750 \\
833,254\end{array}$ & 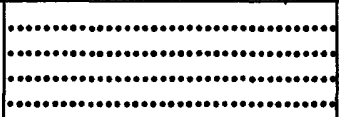 & $\begin{array}{r}44,920 \\
150,260 \\
550,650 \\
1,313,530\end{array}$ \\
\hline $\begin{array}{l}1905 \\
1910 \\
1915\end{array}$ & $\begin{array}{l}1,123,630 \\
1,573,341 \\
2,027,583\end{array}$ & ( & $\begin{array}{l}1,793,600 \\
2,556,200 \\
3,373,320\end{array}$ \\
\hline $\begin{array}{l}1916 \\
1917 \\
1918 \\
1919 \\
1920\end{array}$ & 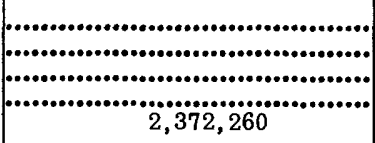 & $\begin{array}{l}46,500,000 \\
46,300,000 \\
42,600,000 \\
28,900,000 \\
30,800,000\end{array}$ & 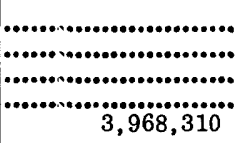 \\
\hline $\begin{array}{l}1921 \\
1922 \\
1923 \\
1924 \\
1925\end{array}$ & $\begin{array}{l}2,416,834 \\
2,424,859 \\
2,440,359 \\
2,394,828 \\
2,460,645\end{array}$ & 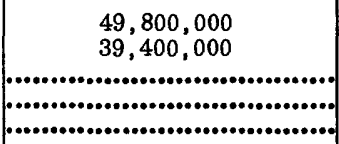 & $\begin{array}{l}4,004,540 \\
4,048,840 \\
4,068,880 \\
4,010,240 \\
4,096,760\end{array}$ \\
\hline $\begin{array}{l}1926 \\
1927 \\
1928 \\
1929 \\
1930\end{array}$ & $\begin{array}{l}2,424,107 \\
2,495,121 \\
2,496,492 \\
2,506,227 \\
2,503,463\end{array}$ & $\begin{array}{r}27,000,000 \\
25,000,000\end{array}$ & $\begin{array}{l}4,049,040 \\
4,162,750 \\
4,162,550 \\
4,179,340 \\
4,173,950\end{array}$ \\
\hline $\begin{array}{l}1931 \\
1932 \\
1933 \\
1934 \\
1935\end{array}$ & $\begin{array}{l}2,447,814 \\
2,515,129 \\
2,535,473 \\
2,436,248 \\
2,542,671\end{array}$ & $\begin{array}{l}20,600,000 \\
34,800,000 \\
32,700,000 \\
29,400,000 \\
25,020,000\end{array}$ & $\begin{array}{l}4,101,550 \\
4,196,100 \\
4,230,960 \\
4,079,900 \\
4,239,000\end{array}$ \\
\hline $\begin{array}{l}1936 \\
1937 \\
1938 \\
1939 \\
1940\end{array}$ & $\begin{array}{l}2,575,719 \\
2,619,679 \\
2,657,810 \\
2,682,664 \\
2,707,010\end{array}$ & $\begin{array}{l}31,460,000 \\
22,310,000 \\
37,540,000 \\
27,320,000 \\
28,810,000\end{array}$ & $\begin{array}{l}4,294,850 \\
4,302,330 \\
4,442,740 \\
4,488,020 \\
4,535,740\end{array}$ \\
\hline $\begin{array}{l}1941 \\
1942 \\
1943 \\
1944 \\
1945 \\
1946\end{array}$ & $\begin{array}{l}2,720,486 \\
2,748,265 \\
2,784,576 \\
2,804,425 \\
2,812,346 \\
2,825,256\end{array}$ & $\begin{array}{l}26,290,000 \\
34,490,000 \\
49,030,000 \\
25,500,000 \\
30,800,000 \\
37,860,000\end{array}$ & $\begin{array}{l}4,560,020 \\
4,610,870 \\
4,676,670 \\
4,713,570 \\
4,727,760 \\
4,750,310\end{array}$ \\
\hline
\end{tabular}


Irrigated area and estimated depletion for the Columbia River below Snake River

\begin{tabular}{|c|c|c|}
\hline Year & $\begin{array}{l}\text { Irrigated area } \\
\text { (acres) }\end{array}$ & $\begin{array}{c}\text { Estimated } \\
\text { depletion } \\
\text { (acre-feet) }\end{array}$ \\
\hline $\begin{array}{l}1860 \\
1870 \\
1880 \\
1890 \\
1900\end{array}$ & $\begin{array}{r}2,020 \\
39,964 \\
125,143 \\
457,710 \\
1,105,557\end{array}$ & $\begin{array}{r}2,300 \\
59,650 \\
187,400 \\
687,440 \\
1,697,650\end{array}$ \\
\hline $\begin{array}{l}1905 \\
1910 \\
1915 \\
1920\end{array}$ & $\begin{array}{l}1,545,186 \\
2,120,073 \\
2,669,905 \\
3,119,274\end{array}$ & $\begin{array}{l}2,428,280 \\
3,416,360 \\
4,407,350 \\
5,175,730\end{array}$ \\
\hline $\begin{array}{l}1921 \\
1922 \\
1923 \\
1924 \\
1925\end{array}$ & $\begin{array}{l}3,165,788 \\
3,191,684 \\
3,199,184 \\
3,155,214 \\
3,217,787\end{array}$ & $\begin{array}{l}5,216,600 \\
5,292,480 \\
5,304,260 \\
5,242,150 \\
5,330,980\end{array}$ \\
\hline $\begin{array}{l}1926 \\
1927 \\
1928 \\
1929 \\
1930\end{array}$ & $\begin{array}{l}3,183,197 \\
3,243,963 \\
3,248,298 \\
3,260,008 \\
3,274,716\end{array}$ & $\begin{array}{l}5,287,060 \\
5,387,770 \\
5,386,790 \\
5,402,740 \\
5,435,310\end{array}$ \\
\hline $\begin{array}{l}1931 \\
1932 \\
1933 \\
1934 \\
1935\end{array}$ & $\begin{array}{l}3,247,198 \\
3,325,323 \\
3,349,275 \\
3,273,397 \\
3,393,571\end{array}$ & $\begin{array}{l}5,411,510 \\
5,519,080 \\
5,564,110 \\
5,452,560 \\
5,634,690\end{array}$ \\
\hline $\begin{array}{l}1936 \\
1937 \\
1938 \\
1939 \\
1940\end{array}$ & $\begin{array}{l}3,412,248 \\
3,483,958 \\
3,524,225 \\
3,550,475 \\
3,577,995\end{array}$ & $\begin{array}{l}5,667,220 \\
5,719,480 \\
5,869,910 \\
5,916,100 \\
5,969,720\end{array}$ \\
\hline $\begin{array}{l}1941 \\
1942 \\
1943 \\
1944 \\
1945 \\
1946\end{array}$ & $\begin{array}{l}3,592,327 \\
3,624,328 \\
3,667,951 \\
3,690,618 \\
3,714,320 \\
3,748,046\end{array}$ & $\begin{array}{l}5,996,100 \\
6,062,350 \\
6,135,050 \\
6,175,550 \\
6,218,140 \\
6,276,290\end{array}$ \\
\hline
\end{tabular}

\section{COLUMBIA RIVER, SNAKE RIVER TO THE DALLES}

\section{WALLA WALLA RIVER}

The Walla Walla River in southern Washington and northern Oregon, is the first tributary to the Columbia River downstream from the Snake River. It drains 1,760 square miles. The river heads in the northern tip of the Blue Mountains and flows generally westward to its confluence with the Columbia River near Wallula, Wash.

Owing to its advantageous location and terrain, the Walla Walla River valley was traversed by the earliest explorers and settlers, and consequently was settled earlier than most other parts of the Oregon Territory. Marcus Whitman established a mission in this valley in 1836. He diverted water from Doan Creek to irrigate garden crops at the mission and soon interested the Indians in doing likewise. 
Irrigation in this basin has been financed by private capital, through small irrigation districts or companies and individual enterprise. The irrigated land is concentrated largely between Walla Walla, Wash. and Freewater, Oreg. and at present totals about 37,000 acres. Of this amount about 22,000 acres are in Washington and 15,000 acres are in Oregon. While most of the area shown in the following table is supplied by direct diversion from small tributaries and branches of the main stream, there is an increasing use of wells and springs for supplemertal supply. The average values in the cited table were compilec primarily from adjudicated rights and records of the irrigation districts and companies, supplemented by local inquiry. Mr. W. C. Mason, assistant watermaster for Umatilla County, Oreg., was helpful in supplying data for the Oregon portion of the valley.

The net consumptive use in the Walla Walla valley was estimated at 1.9 acre-feet per acre, based upon computations by the LowryJohnson method. Return flow from the irrigated lands was assumed to be complete only at the mouth of the river.

Irrigated area and estimated depletion for Walla Walla River basin

\begin{tabular}{l|c|r}
\hline Year & $\begin{array}{c}\text { Irrigated area } \\
\text { (acres) }\end{array}$ & $\begin{array}{r}\text { Estimated } \\
\text { depletion } \\
\text { (acre-feet) }\end{array}$ \\
\hline 1870 & 1,280 & 2,430 \\
1880 & 2,950 & 5,600 \\
1890 & 7,170 & 13,600 \\
1900 & 12,100 & 22,990 \\
1905 & 16,070 & 30,530 \\
1910 & 22,370 & 42,500 \\
1915 & 29,620 & 56,280 \\
1920 & 33,300 & 63,270 \\
1921 & 32,960 & 62,620 \\
1922 & 32,790 & 62,300 \\
1923 & 32,630 & 62,000 \\
1924 & 32,510 & 61,770 \\
1925 & 32,240 & 61,260 \\
1926 & 31,970 & 60,740 \\
1927 & 32,130 & 61,050 \\
1928 & 31,790 & 60,400 \\
1929 & 31,450 & 59,760 \\
1930 & 31,850 & 60,520 \\
1931 & 32,320 & 61,410 \\
1932 & 32,710 & 61,150 \\
1933 & 33,190 & 63,060 \\
1934 & 34,000 & 63,840 \\
1935 & 34,350 & 64,600 \\
1936 & 34,750 & 65,260 \\
1937 & 35,090 & 66,020 \\
1938 & 35,500 & 66,670 \\
1939 & 35,520 & 67,450 \\
1940 & 35,690 & 67,490 \\
1941 & 35,790 & 67,540 \\
1942 & 36,470 & 67,810 \\
1943 & 36,680 & 68,000 \\
1944 & & 68,060 \\
1945 & & 69,290 \\
1946 & & \\
\hline & & \\
\hline
\end{tabular}




\section{UMATILLA RIVER}

The Umatilla River heads in the Blue Mountains of northeastern Oregon and flows generally westward to its confluence with the Columbia River at Umatilla, Oreg. It drains 2,290 square miles. The upper reaches of this basin are heavily forested, but the middle part is largely rolling plains devoted to dry farming. The irrigated area is located primarily on the lower plains near the mouth of the river.

Irrigation in the Umatilla River basin started about 1860, expanded slowly until shortly after 1900 and at presert is about 45,000 acres. In 1905 the Bureau of Reclamation started work on its Umatilla project which comprises two operating divisions -namely, the East or Hermiston and the West Extension divisions. The project includes 13,000 acres near Umatilla. Storage of flood waters is provided in two reservoirs, Cold Springs and McKay Creek Reservoirs. The Cold Springs Reservoir was built in 1907, with a capacity of 50,000 acre-feet. It is supplied with water from the Umatilla River by means of a feeder canal which diverts water near Echo. The McKay Creek Reservoir was built during 1923-26 on McKay Creek near Pendleton, and has a capacity of 73,660 acre-feet. Besides furnishing water to the Hermiston and West Extension divisions, these reservoirs supply some water to the private irrigation districts below Pendleton.

Nine irrigation districts have been developed by private capital, the Stanfield Irrigation district and the Westland Irrigation District being the two largest. There are also smallirrigation developments on Birch and Butter Creeks. There is a small amount of water diverted from upper reaches of the John Day River into Butter Creek. The following table shows the distribution of the irrigated area within the basin. The values shown in this table were compiled largely from records of the Oregon State Engineer and the Bureau of Reclamation: according to Mr. M. B. Bennett, watermaster of Umatilla County, these should be very close to the actual irrigated acreage.

A net consumptive use of 2.0 acre-feet per acre was used for all lands in this basin. Return flow from these lands was estimated to reach the Umatilla River above its confluence with the Columbia. This may be slightly in error because of the irrigated bench lands lying along the Columbia River, but for Frurpose of this report this small bypass has been disregarded. The average net streamflow depletion for the period 1921 through 1945 amounted to 23.2 percent of the yield of this basin. It has remaired essentially constant during this 25 -year period. 
Irrigated area, annual runoff and estimated depletion for Umatilla Rivir basin

\begin{tabular}{|c|c|c|c|c|c|c|}
\hline \multirow{2}{*}{ Year } & \multirow{2}{*}{$\begin{array}{c}\text { McKay Creek } \\
\text { basin }\end{array}$} & \multirow{2}{*}{$\begin{array}{c}\begin{array}{c}\text { Birch Creek } \\
\text { basin }\end{array} \\
\begin{array}{c}\text { Irrigated area } \\
\text { (acres) }\end{array}\end{array}$} & \multirow{2}{*}{\begin{tabular}{|c|}
$\begin{array}{c}\text { Butter Creek } \\
\text { basin }\end{array}$ \\
$\begin{array}{c}\text { Irrigated area } \\
\text { (acres) }\end{array}$
\end{tabular}} & \multicolumn{3}{|c|}{ Umatilla River near Unatilla, Oreg. } \\
\hline & & & & $\begin{array}{l}\text { Irrigated area } \\
\text { (acres) }\end{array}$ & $\begin{array}{c}\text { Runoff } \\
\text { (acre-feet) }\end{array}$ & $\begin{array}{l}\text { Estimated } \\
\text { depletion } \\
\text { (acre-feet) }\end{array}$ \\
\hline $\begin{array}{l}1860 \\
1870 \\
1880 \\
1890 \\
1900\end{array}$ & $\begin{array}{r}30 \\
60 \\
834\end{array}$ & $\begin{array}{r}36 \\
691 \\
1,365 \\
2,039\end{array}$ & $\begin{array}{l}1,111 \\
2,084 \\
2,901 \\
5,150\end{array}$ & $\begin{array}{r}23 \\
1,226 \\
3,785 \\
5,669 \\
12,990\end{array}$ & • & $\begin{array}{r}50 \\
2,450 \\
7,570 \\
11,340 \\
25,980\end{array}$ \\
\hline $\begin{array}{l}1904 \\
1905 \\
1906\end{array}$ & 1,777 & 2,525 & 5,475 & 18,794 & $\begin{array}{l}805,40 ? \\
208,00 ? \\
435,00 ?\end{array}$ & 37,590 \\
\hline 1907 & $\because$ & & & & 619,00 ? & 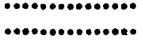 \\
\hline $\begin{array}{l}1908 \\
1909\end{array}$ & •............... & ............ & & & $299,00 ?$ & •.............. \\
\hline 1910 & 1,399 & 3,007 & 5,475 & 25,712 & $477,00 ?$ & 51,420 \\
\hline 1911 & & & & & $204,00 ?$ & "....... \\
\hline $\begin{array}{l}1912 \\
1913\end{array}$ & $\cdots \cdots \cdots \cdots \cdots$ & $\cdots \cdots \cdots \cdots \cdots \bullet$ & - & & $620,00 ?$ & $\cdots \cdots \cdots$ \\
\hline 1914 & & & & & $\begin{array}{l}631,00 ? \\
327,00 \text { ? }\end{array}$ & 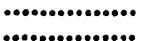 \\
\hline 1915 & 1,627 & 3,612 & 5,475 & 36,491 & 169,007 & 72,980 \\
\hline 1916 & & & & & $667,00 ?$ & a.... \\
\hline $\begin{array}{l}1917 \\
1918\end{array}$ & & $\cdots \cdot \cdots$ & $\cdots$ & & $770,00^{\wedge}$ & $\bullet \cdots \cdots \cdots \cdots \cdots \cdots$ \\
\hline 1919 & & & & & $\begin{array}{l}456,00 ? \\
265,00 ?\end{array}$ & "............... \\
\hline 1920 & 1,686 & $\ddot{3}, 707$ & 5,618 & 43,096 & $445,00 ?$ & 86,190 \\
\hline $\begin{array}{l}1921 \\
1922 \\
1923 \\
1924 \\
1925\end{array}$ & $\begin{array}{l}1,686 \\
1,686 \\
1,710 \\
1,819 \\
1,937\end{array}$ & $\begin{array}{l}3,717 \\
3,722 \\
3,722 \\
3,750 \\
3,771\end{array}$ & $\begin{array}{l}5,618 \\
5,618 \\
5,618 \\
5,618 \\
5,618\end{array}$ & $\begin{array}{l}43,132 \\
43,189 \\
43,244 \\
43,416 \\
43,555\end{array}$ & $\begin{array}{l}578,00 \text { ר } \\
475,00 \text { า } \\
251,00 \text { ) } \\
249,00 \text { ) } \\
308,000\end{array}$ & $\begin{array}{l}86,260 \\
86,380 \\
86,490 \\
86,830 \\
87,110\end{array}$ \\
\hline $\begin{array}{l}1926 \\
1927 \\
1928 \\
1929 \\
1930\end{array}$ & $\begin{array}{l}2,072 \\
2,193 \\
2,193 \\
2,193 \\
2,193\end{array}$ & $\begin{array}{l}3,771 \\
3,771 \\
3,869 \\
3,869 \\
3,869\end{array}$ & $\begin{array}{l}5,663 \\
5,663 \\
5,663 \\
5,663 \\
5,663\end{array}$ & $\begin{array}{l}43,735 \\
43,858 \\
43,956 \\
44,018 \\
44,018\end{array}$ & $\begin{array}{l}174,00 \text { ? } \\
338,00 \text { ? } \\
613,00 \text { ? } \\
212,007 \\
160,00 \text { ? }\end{array}$ & $\begin{array}{l}84,470 \\
87,720 \\
87,910 \\
88,040 \\
88,040\end{array}$ \\
\hline $\begin{array}{l}1931 \\
1932 \\
1933 \\
1934 \\
1935\end{array}$ & $\begin{array}{l}2,193 \\
2,193 \\
2,193 \\
2,193 \\
2,193\end{array}$ & $\begin{array}{l}3,869 \\
3,869 \\
3,869 \\
3,869 \\
3,907\end{array}$ & $\begin{array}{l}5,663 \\
5,663 \\
5,663 \\
5,663 \\
5,663\end{array}$ & $\begin{array}{l}44,018 \\
44,078 \\
44,078 \\
44,078 \\
44,150\end{array}$ & $\begin{array}{l}197,00 \text { ? } \\
472,00 ? \\
332,00 ? \\
183,30 \text { ? } \\
149,20 ?\end{array}$ & $\begin{array}{l}88,040 \\
88,160 \\
88,160 \\
88,160 \\
88,300\end{array}$ \\
\hline $\begin{array}{l}1936 \\
1937 \\
1938 \\
1939 \\
1940\end{array}$ & $\begin{array}{l}2,203 \\
2,203 \\
2,203 \\
2,203 \\
2,229\end{array}$ & $\begin{array}{l}3,945 \\
4,065 \\
4,065 \\
4,070 \\
4,070\end{array}$ & $\begin{array}{l}5,663 \\
5,663 \\
5,663 \\
5,663 \\
5,663\end{array}$ & $\begin{array}{l}44,241 \\
44,601 \\
44,721 \\
44,726 \\
44,786\end{array}$ & $\begin{array}{l}234,80 ? \\
170,70^{?} \\
226,70^{?} \\
203,10 \text { ? } \\
223,90 ?\end{array}$ & $\begin{array}{l}88,481 \\
89,200 \\
89,440 \\
89,450 \\
89,570\end{array}$ \\
\hline $\begin{array}{l}1941 \\
1942 \\
1943 \\
1944 \\
1945 \\
1946\end{array}$ & $\begin{array}{l}2,229 \\
2,229 \\
2,229 \\
2,229 \\
2,229 \\
2,229\end{array}$ & $\begin{array}{l}4,091 \\
4,091 \\
4,091 \\
4,091 \\
4,091 \\
4,091\end{array}$ & $\begin{array}{l}5,663 \\
5,663 \\
5,663 \\
5,663 \\
5,784 \\
5,784\end{array}$ & $\begin{array}{l}44,807 \\
44,807 \\
44,807 \\
44,807 \\
45,048 \\
45,134\end{array}$ & $\begin{array}{l}123,60^{n} \\
386,30^{2} \\
558,60^{?} \\
172,60^{n} \\
303,70^{2} \\
385,10 \text { ? }\end{array}$ & $\begin{array}{l}89,610 \\
89,610 \\
89,610 \\
89,610 \\
90,100 \\
90,270\end{array}$ \\
\hline
\end{tabular}




\section{WLLOW CREEK}

Willow Creek drains approximately 900 square miles of land lying between the John Day and the Umatilla watersheds in northcentral Oregon. The irrigation projects have been minor and have changed little since about 1910. Most of the lands are scattered along both the main stem and tributaries and have been developed by private enterprise. During the autumn there is insufficient water to supply all water rights in full. In the "Review Report of Columbia River and Tributaries," Appendix, K, "Minor Tributaries below the Yakima River, " by the Corps of Engireers, Department of the Army, October 1, 1948, the following statement is made: "Owners of most of these lands have established rights to the waters of the streams for irrigation, but the water supply is so undependable that only about 60 percent of these lands are irrigated. In the valley below Heppner a total of 69 separate diversion rights for the irrigation of 3,760 acres has been established." The steep gradient of the river makes it easy tc construct and operate small canals suitable for supplying individual farms. There is no storage reservoir within the basin. A small amount of water is diverted into the upper reaches of Willow Creek from the John Day River basin to the south.

There is nogaging station within the Willow Creek basin so that the total irrigated acreage shown in the following table is not subdivided. A netconsumptive use of 1.7 acre-feet per acre was estimated for the irrigated lands. Return flow is assumed to be recaptured above the mouth of the creek. 
Irrigated area and estimated depletion for Willow Creek basir

\begin{tabular}{|c|c|c|}
\hline Year & $\begin{array}{l}\text { Irrigated area } \\
\text { (acres) }\end{array}$ & $\begin{array}{c}\text { Estimated } \\
\text { depletion } \\
\text { (acre-feet) }\end{array}$ \\
\hline $\begin{array}{l}1870 \\
1880 \\
1890 \\
1900\end{array}$ & $\begin{array}{r}146 \\
1,137 \\
2,127 \\
4,499\end{array}$ & $\begin{array}{r}250 \\
1,930 \\
3,610 \\
7,650\end{array}$ \\
\hline $\begin{array}{l}1905 \\
1910 \\
1915 \\
1920\end{array}$ & $\begin{array}{l}5,679 \\
6,253 \\
6,458 \\
6,491\end{array}$ & $\begin{array}{r}9,650 \\
10,630 \\
10,980 \\
11,030\end{array}$ \\
\hline $\begin{array}{l}1921 \\
1922 \\
1923 \\
1924 \\
1925\end{array}$ & $\begin{array}{l}6,505 \\
6,519 \\
6,573 \\
6,615 \\
6,630\end{array}$ & $\begin{array}{l}11,060 \\
11,080 \\
11,170 \\
11,250 \\
11,270\end{array}$ \\
\hline $\begin{array}{l}1926 \\
1927 \\
1928 \\
1929 \\
1930\end{array}$ & $\begin{array}{l}6,662 \\
6,667 \\
6,687 \\
6,687 \\
6,719\end{array}$ & $\begin{array}{l}11,320 \\
11,330 \\
11,370 \\
11,370 \\
11,420\end{array}$ \\
\hline $\begin{array}{l}1931 \\
1932 \\
1933 \\
1934 \\
1935\end{array}$ & $\begin{array}{l}6,719 \\
6,719 \\
6,719 \\
6,850 \\
6,870\end{array}$ & $\begin{array}{l}11,420 \\
11,420 \\
11,420 \\
11,640 \\
11,680\end{array}$ \\
\hline $\begin{array}{l}1936 \\
1937 \\
1938 \\
1939 \\
1940\end{array}$ & $\begin{array}{l}6,887 \\
6,887 \\
6,887 \\
6,887 \\
6,887\end{array}$ & $\begin{array}{l}11,710 \\
11,710 \\
11,710 \\
11,710 \\
11,710\end{array}$ \\
\hline $\begin{array}{l}1941 \\
1942 \\
1943 \\
1944 \\
1945 \\
1946\end{array}$ & $\begin{array}{l}6,887 \\
6,887 \\
6,887 \\
6,887 \\
6,887 \\
6,887\end{array}$ & $\begin{array}{l}11,710 \\
11,710 \\
11,710 \\
11,710 \\
11,710 \\
11,710\end{array}$ \\
\hline
\end{tabular}

\section{JOHN DAY RIVER}

The earliest settlers in the John Day River basin were cattlemen who came to the valley near Prairie City about 1860. Soon after, the area had a short-lived gold rush following a discovery of gold near Canyon City. Settlement of the basin has been slow owing to lack of adequate transportation facilities. Irrigation, which has increased with the growth of the livestock industry, is used primarily to grow forage crops for local consumption and has been developed mostly by private enterprise. The earlier projects were centered around Prairie City and are reported to have begun shortly after 1860. Most of the irrigation is done or the upper reaches of the river, where the agricultural land lies close enough to the stream to permit simple diversion works. The lower reaches of the river flow mostly through canyons, and there the land, in general, is unsuitable for farming. 
There are no large reservoirs within the basin and most of the lands served by existing irrigation ditches would benefit from supplemental stored water. Two small diversions carry water from the John Day River basin at present - - one into Willow Creek and the other into Butter Creek, a tributary of the Umatilla River, However, comparatively small amounts of water are carried. The Teel project included plans for an interbasin diversion of water from Camas Creek into Butter Creek by means of a long canal and tunnel with a capacity of 350 second-feet; however, this project was closed down because of financial difficulties and has never been completed.

The irrigated acreages which are shown in the following table are based primarily upon the records of Oregon State Engineer, which agree fairly well with the reports published by the Bureau of the Census of 1939. It is believed that these two records represent the amount of land which ordinarily is irrigated during the years of sufficient water supply. Tabulation of irrigated area have been subdivided to show the area above the gaging staticns, North Fork John Day River near Monument and John Day River at McDonald Ferry. Return flow from the lands above these two gaging stations is assumed to reach the main stem of the river above the respective stations. In this basin, net consumptive use of 1.7 acre-feet per acre was estimated for the irrigated lands. The average streamflow depletion for the period 1921 through 1945 amounts to 6.0 percent of the yield of this basin above McDonald Ferry. It has increased only slightly during this period, and in 1946 the net depletion represents 6.3 percent of the long-term yield of this basin. 
Irrigated area, annual runoff, and estimated depletion for John Day River basin

\begin{tabular}{|c|c|c|c|c|}
\hline \multirow{2}{*}{ Year } & \multirow{2}{*}{$\begin{array}{c}\text { North Fork } \\
\text { John Day River } \\
\text { at Monument, Oreg. } \\
\begin{array}{c}\text { Irrigated area } \\
\text { (acres) }\end{array}\end{array}$} & \multicolumn{3}{|c|}{ John Day River at McDonald Ferry, Oreg. } \\
\hline & & $\begin{array}{l}\text { Irrigated area } \\
\text { (acres) }\end{array}$ & $\begin{array}{c}\text { Runoff } \\
\text { (acre-feet) }\end{array}$ & $\begin{array}{c}\text { Estimated } \\
\text { depletion } \\
\text { (acre-feet) }\end{array}$ \\
\hline $\begin{array}{l}1860 \\
1870 \\
1880 \\
1890 \\
1900\end{array}$ & $\begin{array}{r}74 \\
406 \\
1,751 \\
\\
2,162\end{array}$ & $\begin{array}{r}85 \\
3,891 \\
11,613 \\
23,018 \\
28,261\end{array}$ & 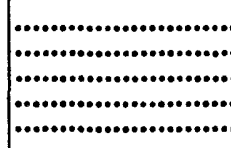 & $\begin{array}{r}140 \\
6,610 \\
19,740 \\
39,130 \\
48,040\end{array}$ \\
\hline $\begin{array}{l}1905 \\
1906 \\
1907 \\
1908 \\
1909 \\
1910\end{array}$ & 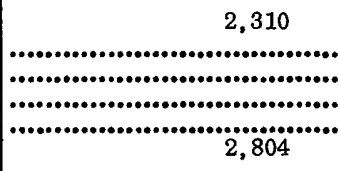 & (148 & $\begin{array}{c}\ldots \ldots \ldots \ldots \\
1,430,000 \\
2,100,000 \\
981,800 \\
992,000 \\
1,500,000\end{array}$ & $\begin{array}{r}54,410 \\
\cdots \ldots \ldots \ldots \cdots \cdots \cdots \cdots \cdots \\
\cdots \cdots \cdots \cdots \cdots \cdots \cdots \cdots \cdots \\
\cdots \cdots \cdots \cdots \cdots \cdots \cdots \cdots \cdots \\
\cdots \cdots \cdots \cdots \cdots\end{array}$ \\
\hline $\begin{array}{l}1911 \\
1912 \\
1913 \\
1914 \\
1915\end{array}$ & $\cdots$ & (4) & $\begin{array}{r}961,000 \\
2,410,000 \\
1,770,000 \\
1,520,000 \\
729,000\end{array}$ & ( \\
\hline $\begin{array}{l}1916 \\
1917 \\
1918 \\
1919 \\
1920\end{array}$ & ( & (n) & $\begin{array}{l}2,380,000 \\
2,250,000 \\
1,370,000 \\
1,280,000 \\
1,520,000\end{array}$ & 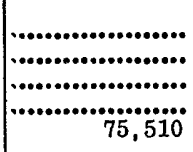 \\
\hline $\begin{array}{l}1921 \\
1922 \\
1923 \\
1924 \\
1925\end{array}$ & $\begin{array}{l}3,642 \\
3,652 \\
3,692 \\
3,702 \\
3,714\end{array}$ & $\begin{array}{l}44,500 \\
44,731 \\
44,971 \\
45,254 \\
45,300\end{array}$ & $\begin{array}{r}2,610,000 \\
2,170,000 \\
1,420,000 \\
803,000 \\
1,510,000\end{array}$ & $\begin{array}{l}75,650 \\
76,040 \\
76,450 \\
76,930 \\
77,010\end{array}$ \\
\hline $\begin{array}{l}1926 \\
1927 \\
1928 \\
1929 \\
1930\end{array}$ & $\begin{array}{l}3,726 \\
4,002 \\
4,177 \\
4,248 \\
4,272\end{array}$ & $\begin{array}{l}45,511 \\
46,615 \\
47,149 \\
47,370 \\
47,598\end{array}$ & $\begin{array}{r}769,000 \\
1,620,000 \\
1,930,000 \\
974,000 \\
531,000\end{array}$ & $\begin{array}{l}77,370 \\
79,250 \\
80,150 \\
80,530 \\
80,920\end{array}$ \\
\hline $\begin{array}{l}1931 \\
1932 \\
1933 \\
1934 \\
1935\end{array}$ & $\begin{array}{l}4,308 \\
4,308 \\
4,321 \\
4,338 \\
4,346\end{array}$ & $\begin{array}{l}47,657 \\
47,710 \\
47,768 \\
47,835 \\
47,993\end{array}$ & $\begin{array}{r}579,000 \\
1,650,000 \\
1,160,000 \\
461,500 \\
665,300\end{array}$ & $\begin{array}{l}81,020 \\
81,110 \\
81,210 \\
81,320 \\
81,590\end{array}$ \\
\hline $\begin{array}{l}1936 \\
1937 \\
1938 \\
1939 \\
1940\end{array}$ & $\begin{array}{l}4,351 \\
4,378 \\
4,378 \\
4,530 \\
4,574\end{array}$ & $\begin{array}{l}48,092 \\
48,183 \\
48,723 \\
49,026 \\
49,220\end{array}$ & $\begin{array}{r}876,600 \\
1,057,000 \\
1,615,000 \\
943,200 \\
1,026,000\end{array}$ & $\begin{array}{l}81,760 \\
81,910 \\
82,830 \\
83,340 \\
83,670\end{array}$ \\
\hline $\begin{array}{l}1941 \\
1942 \\
1943 \\
1944 \\
1945 \\
1946\end{array}$ & $\begin{array}{l}4,624 \\
4,626 \\
4,642 \\
4,699 \\
4,739 \\
4,894\end{array}$ & $\begin{array}{l}49,378 \\
49,411 \\
49,443 \\
49,626 \\
49,742 \\
50,307\end{array}$ & $\begin{array}{r}1,157,000 \\
2,041,000 \\
2,443,000 \\
623,300 \\
1,220,000 \\
1,559,000\end{array}$ & $\begin{array}{l}83,940 \\
84,000 \\
84,050 \\
84,360 \\
84,560 \\
85,520\end{array}$ \\
\hline
\end{tabular}




\section{DESCHUTES RIVER}

Irrigation in Deschutes River basin has increased gradually since about 1880 with the largest spurts from 1890 to 1.910 and after 1945. This basin has extensive irrigable land, but because the soil commonly is very permeable, a large part of it ras been without an adequate water supply. The earliest irrigation ventures were started as Carey Act projects, the largest of which are the Crook County Improvement District, the Arnold Irrigation Co., the Central Oregon Irrigation District and the Swalley Irrigation Co. All are in the upper valley near Bend, Oreg.

The Crooked River is the largest tributary of the Deschutes and its irrigated area is about 38,000 acres out of a total of 145, 000 irrigated acres in all the Deschutes River basin. Most of the irrigation in the valley of Crooked River is in the headwater area above Ochoco Creek. The only large irrigation project in the valley, the Ochoco Irrigation Co. was developed by private capital in 1921. It started with an irrigable area of 22,000 acres but later this was reduced to 8,500 acres because of water shortages. (See table on page 118.)

Smaller tributaries along the lower Deschutes River, namely Squaw and Tumalo Creeks, have scattered tracts of irrigated land but their total irrigated area is small in comparison with that of the main valley. In recent years a small amount of land has been irrigated in the White River basinby three small ditch companies. There is very little chance for irrigation in the Metolius River basin.

The irrigated acreages listed in the tables on pp. 119, 120 have been based on adjudicated water rights and the records of the irrigation districts within the Deschutes River basin. They were derived independently of, but agree very closely with the values given in reports by the Geological Survey (Henshaw, Lewis, and McCaustland, 1914), and the Federal Power Commission (Henry, Cavanaugh and Henshaw, 1922).

This basin is so subdivided topographically as to defire quite closely the points at which most of the return flow from the irrigated areas might be expected to be recaptured by the Deschutes River. Along the main stem, the return flow from all the irrigated area above the gaging station near Madras (see table on p. 119 ) is assumed to be recaptured above that station; return flow from the Crooked River basin, all above the gaging station near the mouth of that tributary near Culver, and from the areas along the other tributaries, all above the mouth of the main stem and and above the gaging station at Moody near Biggs. (See table on p. 120). A net consumptive use of 1.7 acre-feet per acre has been used in estimating streamflow depletion. 
The north unit of the Deschutes project is currently irrigating land located north of the Crooked River along the east kank of the Deschutes River. The first large scale irrigation by this unit started in 1946 and has been expanding since that time. The return flow from this area is assumed to be down Willow and Mud Spring Creeks to the Deschutes River. Probably about half this area drains to the main stem above the station at Madras and the remainder above the station near Biggs.

In the Deschutes River basin streamflow depletion is not a very important factor. During the period 1921 through 1945, the average depletion above the gaging station Crooked River near Culver, has amounted to only 5.2 percent of the yield of the basin. There has been only a slight increase during the base poriod, and in 1946, it amounted to 5.7 percent of the long-term yield. The average depletion during the period 1924 through 1946, has amounted to 3.8 percent of the yield of the basin above the gaging station near Madras. During 1945 and 1946, a large increase in irrigated area took place, and for 1946, the depletion amounted to 5.7 percent of the long-term yield. The average depletion above the station at Moody during the period 1921 thrcugh 1945, amounted to 3.6 percent. For 1946, the depletion amounted to 5.1 percent of the long-term yield. 
Irrigated area, annual runoff and estimated depletion for Crooked River basin

\begin{tabular}{|c|c|c|c|c|c|}
\hline \multirow{2}{*}{ Year } & \multirow{2}{*}{$\begin{array}{c}\begin{array}{c}\text { Crooked River } \\
\text { near } \\
\text { Post, Oreg. }\end{array} \\
\begin{array}{c}\text { Irrigated area } \\
\text { (acres) }\end{array}\end{array}$} & \multirow{2}{*}{$\begin{array}{c}\begin{array}{c}\text { Crooked River } \\
\text { above Hoff man } \\
\text { Dam near }\end{array} \\
\text { Prineville, Oreg. } \\
\begin{array}{c}\text { Irrigated area } \\
\text { (acres) }\end{array}\end{array}$} & \multicolumn{3}{|c|}{ Crooked River near Culver, Oreg. } \\
\hline & & & $\begin{array}{l}\text { Irrigated area } \\
\text { (acres) }\end{array}$ & $\begin{array}{c}\text { Runoff } \\
\text { (acre-feet) }\end{array}$ & $\begin{array}{c}\text { Estimated } \\
\text { depletion } \\
\text { (acre-feet) }\end{array}$ \\
\hline $\begin{array}{l}1870 \\
1880 \\
1890 \\
1900\end{array}$ & $\begin{array}{r}530 \\
2,340 \\
5,900 \\
9,300\end{array}$ & $\begin{array}{r}660 \\
2,940 \\
7,500 \\
11,600\end{array}$ & $\begin{array}{r}1,190 \\
5,309 \\
13,556 \\
21,124\end{array}$ & 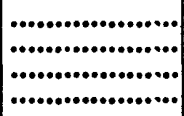 & $\begin{array}{r}2,020 \\
9,030 \\
23,040 \\
35,910\end{array}$ \\
\hline $\begin{array}{l}1905 \\
1910 \\
1915\end{array}$ & $\begin{array}{l}10,800 \\
11,800 \\
12,500\end{array}$ & $\begin{array}{l}13,500 \\
14,600 \\
15,800\end{array}$ & $\begin{array}{l}24,414 \\
26,746 \\
28,217\end{array}$ & 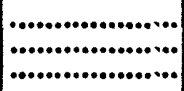 & $\begin{array}{l}41,500 \\
45,470 \\
47,970\end{array}$ \\
\hline $\begin{array}{l}1918 \\
1919\end{array}$ & & & & $\begin{array}{r}958,000 \\
1,000,000\end{array}$ & \\
\hline 1920 & 13,820 & 17,200 & 31,457 & 920,000 & 53,480 \\
\hline $\begin{array}{l}1921 \\
1922 \\
1923 \\
1924 \\
1925\end{array}$ & $\begin{array}{l}13,900 \\
14,000 \\
14,100 \\
14,200 \\
14,250\end{array}$ & $\begin{array}{l}17,300 \\
17,400 \\
17,600 \\
17,700 \\
17,800\end{array}$ & $\begin{array}{l}31,683 \\
31,752 \\
32,122 \\
32,177 \\
32,217\end{array}$ & $\begin{array}{r}1,170,000 \\
1,041,000 \\
999,000 \\
902,000 \\
1,076,000\end{array}$ & $\begin{array}{l}53,860 \\
53,980 \\
54,610 \\
54,700 \\
54,770\end{array}$ \\
\hline $\begin{array}{l}1926 \\
1927 \\
1928 \\
1929 \\
1930\end{array}$ & $\begin{array}{l}14,300 \\
14,300 \\
14,300 \\
14,400 \\
14,400\end{array}$ & $\begin{array}{l}17,800 \\
17,900 \\
18,000 \\
18,000 \\
18,100\end{array}$ & $\begin{array}{l}32,231 \\
32,483 \\
32,564 \\
32,594 \\
32,787\end{array}$ & $\begin{array}{r}928,000 \\
1,110,000 \\
1,076,000 \\
914,000 \\
923,000\end{array}$ & $\begin{array}{l}54,790 \\
55,220 \\
55,360 \\
55,410 \\
55,740\end{array}$ \\
\hline $\begin{array}{l}1931 \\
1932 \\
1933 \\
1934 \\
1935\end{array}$ & $\begin{array}{l}14,500 \\
14,500 \\
14,500 \\
14,600 \\
15,050\end{array}$ & $\begin{array}{l}18,200 \\
18,300 \\
18,400 \\
18,500 \\
18,900\end{array}$ & $\begin{array}{l}32,837 \\
32,995 \\
33,095 \\
33,213 \\
34,133\end{array}$ & $\begin{array}{r}894,000 \\
1,088,000 \\
995,000 \\
894,700 \\
978,700\end{array}$ & $\begin{array}{l}55,820 \\
56,090 \\
56,260 \\
56,460 \\
58,030\end{array}$ \\
\hline $\begin{array}{l}1936 \\
1937 \\
1938 \\
1939 \\
1940\end{array}$ & $\begin{array}{l}15,000 \\
15,000 \\
15,500 \\
15,800 \\
15,900\end{array}$ & $\begin{array}{l}18,900 \\
19,000 \\
19,500 \\
19,800 \\
19,900\end{array}$ & $\begin{array}{l}34,168 \\
34,168 \\
34,203 \\
35,800 \\
36,073\end{array}$ & $\begin{array}{r}1,065,000 \\
1,023,000 \\
1,286,000 \\
977,600 \\
1,087,000\end{array}$ & $\begin{array}{l}58,090 \\
58,090 \\
58,150 \\
60,860 \\
61,320\end{array}$ \\
\hline $\begin{array}{l}1941 \\
1942 \\
1943 \\
1944 \\
1945 \\
1946\end{array}$ & $\begin{array}{l}15,900 \\
16,000 \\
16,000 \\
16,100 \\
16,100 \\
16,200\end{array}$ & $\begin{array}{l}20,000 \\
20,000 \\
20,100 \\
20,200 \\
20,300 \\
20,400\end{array}$ & $\begin{array}{l}36,126 \\
36,126 \\
36,196 \\
36,651 \\
36,688 \\
37,430\end{array}$ & $\begin{array}{r}1,069,000 \\
1,183,000 \\
1,460,000 \\
995,800 \\
1,143,000 \\
1,360,000\end{array}$ & $\begin{array}{l}61,410 \\
61,410 \\
61,530 \\
62,310 \\
62,370 \\
63,630 \\
\end{array}$ \\
\hline
\end{tabular}


Irrigated area, annual runoff and estimated depletion for Deschutes River neä-Madras, Oreg.

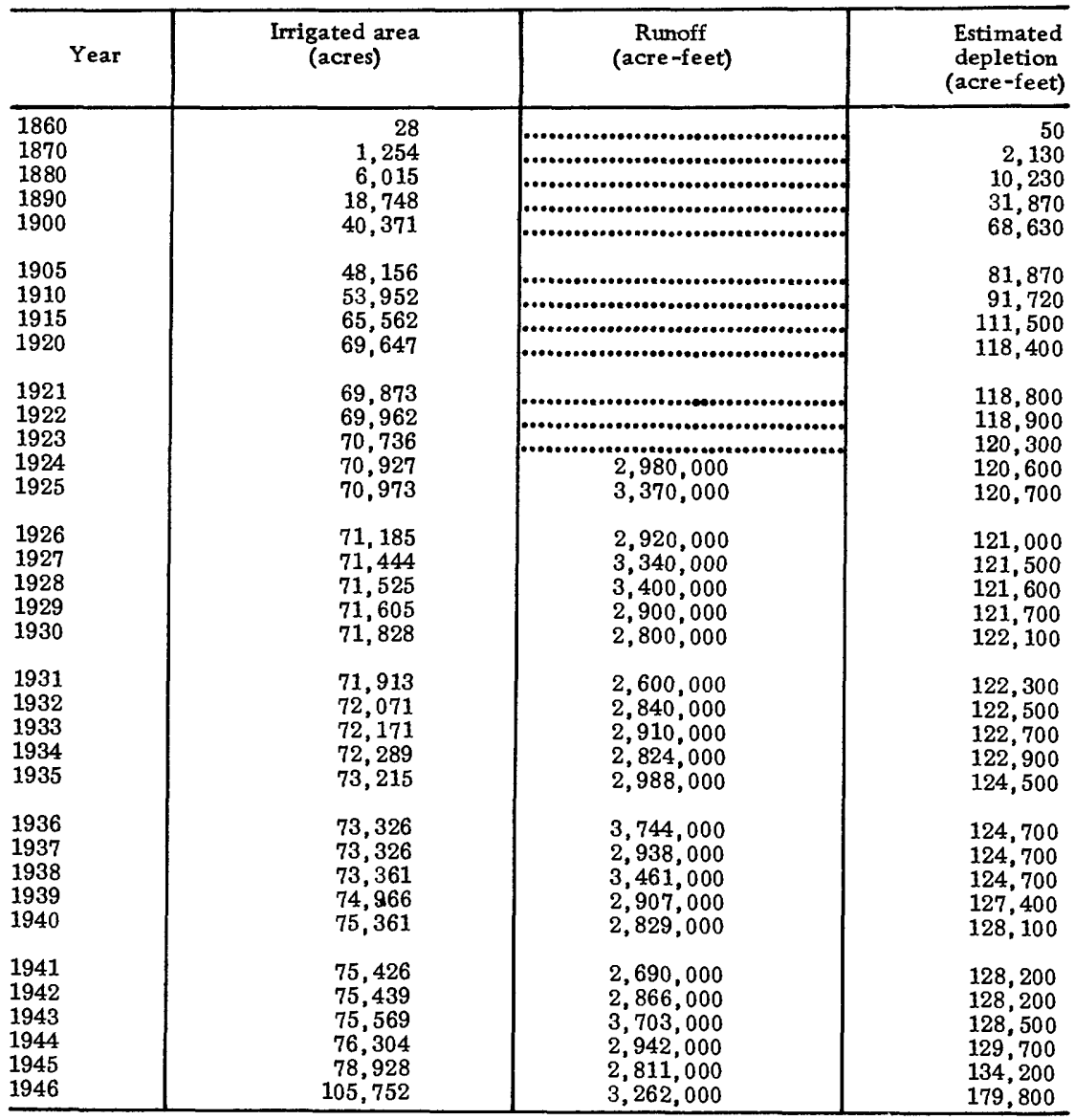




\section{IRRIGATION AND STREAMFLOW DEPLETION IN COLUMBIA RIVER BASIN}

Irrigated area, annual runoff, and estimated depletion for Deschutes River basin

\begin{tabular}{|c|c|c|c|c|}
\hline \multirow{2}{*}{ Year } & \multirow{2}{*}{$\begin{array}{c}\text { White River below } \\
\text { Tygh Valley, Oreg. } \\
\begin{array}{c}\text { Irrigated area } \\
\text { (acres) }\end{array}\end{array}$} & \multicolumn{3}{|c|}{ Deschutes River at Moody, near Biges, Oreg. } \\
\hline & & $\begin{array}{l}\text { Irrigated area } \\
\text { (acres) }\end{array}$ & $\begin{array}{c}\text { Runoff } \\
\text { (acre-feet) }\end{array}$ & $\begin{array}{l}\text { Estimated } \\
\text { depletion } \\
\text { (acre-feet) }\end{array}$ \\
\hline $\begin{array}{l}1860 \\
1870 \\
1880 \\
1890 \\
1900\end{array}$ & $\begin{array}{r}734 \\
1,762 \\
1,927 \\
\\
2,203\end{array}$ & $\begin{array}{r}28 \\
2,072 \\
8,415 \\
22,679 \\
46,197\end{array}$ & $\mid$ & $\begin{array}{r}50 \\
3,520 \\
14,300 \\
38,550 \\
78,530\end{array}$ \\
\hline $\begin{array}{l}1905 \\
1910\end{array}$ & $\begin{array}{l}2,870 \\
3,370\end{array}$ & $\begin{array}{l}54,815 \\
61,140\end{array}$ & $\cdots, 690,000$ & $\begin{array}{r}93,190 \\
103,900\end{array}$ \\
\hline $\begin{array}{l}1911 \\
1912 \\
1913 \\
1914 \\
1915\end{array}$ & 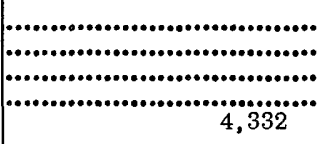 & $\mid \cdots \ldots \ldots \ldots \ldots \ldots \ldots \ldots \ldots \ldots \ldots \ldots \ldots$ & $\begin{array}{l}4,420,000 \\
5,100,000 \\
4,840,000 \\
4,410,000 \\
3,600,000\end{array}$ & 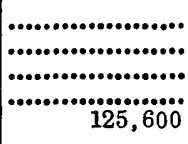 \\
\hline $\begin{array}{l}1916 \\
1917 \\
1918 \\
1919 \\
1920\end{array}$ & 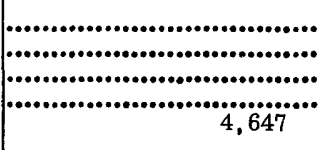 & $\mid \ldots \ldots \ldots \ldots \ldots \ldots \ldots \ldots \ldots \ldots \ldots \ldots \ldots \ldots$ & $\begin{array}{l}5,160,000 \\
4,510,000 \\
4,200,000 \\
3,950,000 \\
3,820,000\end{array}$ & ( \\
\hline $\begin{array}{l}1921 \\
1922 \\
1923 \\
1924 \\
1925\end{array}$ & $\begin{array}{l}4,687 \\
4,743 \\
5,041 \\
5,151 \\
5,270\end{array}$ & $\begin{array}{l}79,012 \\
79,157 \\
80,229 \\
80,530 \\
80,772\end{array}$ & $\begin{array}{l}4,800,000 \\
4,730,000 \\
4,410,000 \\
3,560,000 \\
4,270,000\end{array}$ & $\begin{array}{l}134,300 \\
134,600 \\
136,400 \\
136,900 \\
137,300\end{array}$ \\
\hline $\begin{array}{l}1926 \\
1927 \\
1928 \\
1929 \\
1930\end{array}$ & $\begin{array}{l}5,295 \\
5,430 \\
5,455 \\
6,550 \\
6,560\end{array}$ & $\begin{array}{l}80,959 \\
81,353 \\
81,459 \\
82,634 \\
82,867\end{array}$ & $\begin{array}{l}3,410,000 \\
4,340,000 \\
4,290,000 \\
3,450,000 \\
3,240,000\end{array}$ & $\begin{array}{l}137,600 \\
138,300 \\
138,500 \\
140,500 \\
140,900\end{array}$ \\
\hline $\begin{array}{l}1931 \\
1932 \\
1933 \\
1934 \\
1935\end{array}$ & $\begin{array}{l}6,575 \\
6,724 \\
6,724 \\
6,769 \\
6,769\end{array}$ & $\begin{array}{l}82,967 \\
83,274 \\
83,374 \\
83,537 \\
84,463\end{array}$ & $\begin{array}{l}3,030,000 \\
3,520,000 \\
3,600,000 \\
3,466,000 \\
3,590,000\end{array}$ & $\begin{array}{l}141,000 \\
141,600 \\
141,700 \\
142,000 \\
143,600\end{array}$ \\
\hline $\begin{array}{l}1936 \\
1937 \\
1938 \\
1939 \\
1940\end{array}$ & $\begin{array}{l}6,769 \\
6,769 \\
6,769 \\
6,769 \\
6,909\end{array}$ & $\begin{array}{l}84,574 \\
84,574 \\
84,609 \\
86,214 \\
86,749\end{array}$ & $\begin{array}{l}3,744,000 \\
3,550,000 \\
4,507,000 \\
3,406,000 \\
3,392,000\end{array}$ & $\begin{array}{l}143,800 \\
143,800 \\
143,800 \\
146,600 \\
147,500\end{array}$ \\
\hline $\begin{array}{l}1941 \\
1942 \\
1943 \\
1944 \\
1945 \\
1946 \\
\end{array}$ & $\begin{array}{l}6,909 \\
6,909 \\
7,109 \\
7,109 \\
7,124 \\
7,223 \\
\end{array}$ & $\begin{array}{r}86,814 \\
86,827 \\
87,157 \\
87,892 \\
90,531 \\
117,454 \\
\end{array}$ & $\begin{array}{l}3,111,000 \\
3,496,000 \\
5,113,000 \\
3,409,000 \\
3,363,000 \\
4,150,000 \\
\end{array}$ & $\begin{array}{l}147,600 \\
147,600 \\
148,200 \\
149,400 \\
153,900 \\
199,700 \\
\end{array}$ \\
\hline
\end{tabular}


COLUMBIA RIVER NEAR THE DALLES, OREG.

The gaging station on the Columbia River near The Dalles, Oreg. is the last one where the yield of the Columbia $R$ iver basin may be measured directly. This affords an opportunity to evaluate the aggregate effects of all upstream irrigation or the yield of the basin as shown in the following table. The averago streamflow depletion amounted to 4.7 percent of the yield during the period 1921 through 1945. There has been a slight increase in irrigated area during the base period and the depletion for 1946 represents 5.3 percent of the yield during that period; 7.4 percent of the yield for 1926; and 3.8 percent of the yield for 1928 . These values indicate the magnitude of the depletion, which is represented by the 1946 -stage of irrigation development, in comparison to the average, minimum and maximum yields during the base period.

Thus it can be seen, that from an overall viewpoint, the streamflow depletion that can be attributed to irrigation is not a major part of the yield of the basin. In fact it is relatively small in comparison to the fluctuations caused by the vagaries in climatic conditions. 
122 IRRIGATION AND STREAMFLOW DEPLETION IN COLUMBIA RIVER BASIN

Irrigated area, annual runoff and estinated depletion for the Columbia River near The Dalles, Oreg.

\begin{tabular}{|c|c|c|c|}
\hline Year & $\begin{array}{l}\text { Irrigated area } \\
\text { (acres) }\end{array}$ & $\begin{array}{c}\text { Runoff } \\
\text { (acre-feet) }\end{array}$ & $\begin{array}{c}\text { Estimated } \\
\text { depletion } \\
\text { (acre-feet) }\end{array}$ \\
\hline $\begin{array}{l}1860 \\
1870 \\
1880 \\
1890 \\
1900\end{array}$ & $\begin{array}{r}2,156 \\
48,580 \\
153,042 \\
518,373 \\
1,209=04\end{array}$ & $\mid \begin{array}{c}192,000,000 \\
143,000,000 \\
163,000,000\end{array}$ & $\begin{array}{r}2,590 \\
74,910 \\
236,540 \\
793,670 \\
1,880,840\end{array}$ \\
\hline $\begin{array}{l}1901 \\
1902 \\
1903 \\
1904 \\
1905\end{array}$ & - & $\begin{array}{l}158,000,000 \\
143,000,000 \\
153,000,000 \\
176,000,000 \\
102,000,000\end{array}$ & $\mid \cdots \ldots \ldots \cdots \cdots \cdots \cdots \ldots \ldots \ldots \ldots \ldots \ldots$, \\
\hline $\begin{array}{l}1906 \\
1907 \\
1908 \\
1909 \\
1910\end{array}$ & $\mid$ & $\begin{array}{l}114,000,000 \\
166,000,000 \\
143,000,000 \\
139,000,000 \\
154,000,000\end{array}$ & ( \\
\hline $\begin{array}{l}1911 \\
1912 \\
1913 \\
1914 \\
1915\end{array}$ & 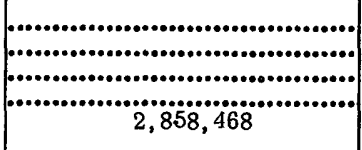 & $\begin{array}{l}136,000,000 \\
134,000,000 \\
155,000,000 \\
135,000,000 \\
107,000,000\end{array}$ & 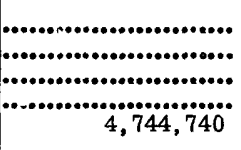 \\
\hline $\begin{array}{l}1916 \\
1917 \\
1918 \\
1919 \\
1920\end{array}$ & 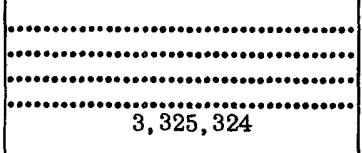 & $\begin{array}{l}173,000,000 \\
152,000,000 \\
148,000,000 \\
125,000,000 \\
115,000,000\end{array}$ & ( \\
\hline $\begin{array}{l}1921 \\
1922 \\
1923 \\
1924 \\
1925\end{array}$ & $\begin{array}{l}3,371,897 \\
3,398,070 \\
3,406,831 \\
3,363,539 \\
3,426,234\end{array}$ & $\begin{array}{r}167,000,000 \\
133,000,000 \\
130,000,000 \\
99,300,000 \\
146,000,000\end{array}$ & $\begin{array}{l}5,586,490 \\
5,662,880 \\
5,676,770 \\
5,615,830 \\
5,704,930\end{array}$ \\
\hline $\begin{array}{l}1926 \\
1927 \\
1928 \\
1929 \\
1930\end{array}$ & $\begin{array}{l}3,392,034 \\
3,454,596 \\
3,459,339 \\
3,472,167 \\
3,487,948\end{array}$ & $\begin{array}{r}85,500,000 \\
149,000,000 \\
168,000,000 \\
96,100,000 \\
95,200,000\end{array}$ & $\begin{array}{l}5,661,560 \\
5,765,420 \\
5,765,120 \\
5,782,940 \\
5,817,110\end{array}$ \\
\hline $\begin{array}{l}1931 \\
1932 \\
1933 \\
1934 \\
1935\end{array}$ & $\begin{array}{l}3,461,879 \\
3,539,814 \\
3,564,404 \\
3,489,297 \\
3,611,047\end{array}$ & $\begin{array}{r}88,400,000 \\
135,000,000 \\
143,000,000 \\
135,100,000 \\
123,200,000\end{array}$ & $\begin{array}{l}5,794,400 \\
5,902,520 \\
5,949,660 \\
5,839,520 \\
6,024,460\end{array}$ \\
\hline $\begin{array}{l}1936 \\
1937 \\
1938 \\
1939 \\
1940\end{array}$ & $\begin{array}{l}3,616,892 \\
3,689,453 \\
3,730,755 \\
3,769,328 \\
3,801,157\end{array}$ & $\begin{array}{r}115,400,000 \\
93,190,000 \\
137,700,000 \\
108,400,000 \\
107,800,000\end{array}$ & $\begin{array}{l}6,058,230 \\
6,112,120 \\
6,264,360 \\
6,314,650 \\
6,369,660\end{array}$ \\
\hline $\begin{array}{l}1941 \\
1942 \\
1943 \\
1944 \\
1945 \\
1946\end{array}$ & $\begin{array}{l}3,835,763 \\
3,846,960 \\
3,892,035 \\
3,915,650 \\
3,943,018 \\
4,004,508\end{array}$ & $\begin{array}{r}94,130,000 \\
129,300,000 \\
150,100,000 \\
86,830,000 \\
109,200,000 \\
142,000,000\end{array}$ & $\begin{array}{l}6,396,500 \\
6,463,080 \\
6,536,620 \\
6,578,690 \\
6,627,700 \\
6,733,180\end{array}$ \\
\hline
\end{tabular}




\section{REFERENCES CITED}

Bancroft, H. H., 1890, History of Washington, Idaho, and Montana, San Francisco, Calif., History Co.

Bark, D., 1916, Experiments on economical use of irrigation water in Idaho: U. S. Dept. Agr., Bull. 339.

Blaney, H. F., 1930, Rainfall penetration and consumptive use of water in Santa Anna River valley and coastal plain; California Dept. of Public Works, Div. of Water Resources Bull. 33, pt. 1, chap. 2.

Blaney, H. F., Ewing, P. A., Istaelsen, O. W., Rohwer, Carl, and Scobey, F. C., Feb. 1938, Water utilization, Upper Rio Grande basin; National Resources Committee, pt. 6, pp. 293-423.

Blaney, H. F., and others, 1942, Consumptive water use and requirements, report of the participating agencies, Pecos River Joint Investigations: National Resources Planning Board, pp. 170-230.

Congressional Documents, 1934, 73d Cong., 1st Sess., H. Doc. 103, vol. 2, p. 879.

Fortier, Samuel, and Young, A. A., 1930, Irrigation requirements of the arid and semiarid lands of the Columbia River basin; U. S. Dept. Agr. Tech. Bull. 200.

Harding, S. T., 1930, Consumptive use of water in irrigation, Progress report of the Duty of Water Committee of the Irrigation Division: Am. Soc. Civ. Eng. Trans., vol 94, pp. 1349-1377.

Hemphill, R. G., 1922, Irrigation in northem Colorado: U. S. Dept. Agr. Bull. 1026.

Henshaw, F. F., Lewis, J. H., McCaustland, E. J., 1914, Deschutes River. Oregon, and its utilization, U. S. Geol. Survey Water-Supply Paper 344.

Henny, D. C., Cavanaugh, J. B., and Henshaw, F. F., 1922, Report to the Federal Power Commission on uses of Deschutes River, Oregon.

Lee, C. H., 1912, An intensive study of the water resources of a part of Owens Valley, Calif.: U. S. Geol. Survey Water-Supply Paper 294.

Lowry, R. L., J r., and Johnson, A. F., 1942, Consumptive use of water fior agriculture: Am. Soc. Civ. Eng. Trans., vol. 107, pp. 1243-1266.

National Resources Planning Board, June 1943, State water law in the divelopment of the West, Report to the Water Resources Committee by its subcommitten on state water law.

Parker, G. L., and Storey, F. B., 1915, Water powers of the Cascade Kange; Part 3, Yakima River basin: U. S. Geol. Survey Water-Supply Paper 369.

Parshall, R. L., 1930, Experiments to determine rate of evaporation from saturated soils and river-bed sands: Am. Soc. Civ. Eng. Trans., vol. 94, pp. 961-972.

Powers, W. L., 1914, Irrigation and soil-moisture investigations in western Oregon: Oregon Agr. Coll. Exp. Sta. Bull. 122.

140 .

Snelson, W. H., 1922, Irrigation practice and water requirements for crops in Alberta: Canada Dept. of Interior, Irrigation Series, Bull. 6.

U. S. Bureau of Reclamation, Dec. 1948, Reclamation Project Data.

White, W. N., 1932, A method of estimating ground-water supplies based on discharge by plants and evaporation from soil: U. S. Geol. Survey Water-Supply Paper 659-A, pp. $1-106$.

Widtsoe, J. A., and Merrill, L. A., 1912, The yields of crops with different quantities of water: Utah Agr. Exp. Sta. Bull. 117. 


\section{INDEX}

Appropriation doctrine.

Big Lost River.

Big Wood River .................................

Boise River.

Bruneau River 00000000

Bunt River....

Carey Act of 1894

Chelan River

Clark Fork, above Missoula, Mont ...... at St. Regis, Mont .......................

below Missoula, Mont ....................

Clarkston to Oxbow, Snake River........

Clearwater River .............................

Columbia River, at Trinidad, Wash ... at Grand Coulee Dam, Wash............ at the international boundary .......... between Yakima River and Snake River ......................................

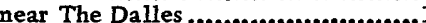

Colville River ....................................

Consumptive use, coefficient........... definition of ............................... estimates of average ..................... formulas for comparison of

net (tabl

Crab Creek

Deschutes River

$116-120$

Earliest irrigation

Entiat River

Evaporation

Field plots

Flathead River, above Flathead Lake

below Flathead Lake .......................

Formula for average consumptive

use.

Gaging stations, list of

Grand Coulee Dam, Columbia

River at

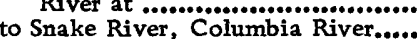

Grand Ronde River...........................

Heise, Snake River above ...................

Heise to King Hill

Henrys Fork

Iistory of irrigation in the basin, earliest irrigation.

rate of expansion.............................. transportation

13-14

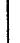

Page

Imnaha River............................... 97-98

International boundary, Colunbia

River at....................................

International boundary to Grard

Coulee Dam, Columbia River..... $\quad 36-40$

John Day River ........................... 113-115

Kettle River............................. 36-37

King Hill, Snake River at.............. 69-71

King Hill, (Heise to, Snake River ..... 62-66

King Hill $\leq 0$ Murphy, Snake

River.................................. 72-90

King Hill to Neeley, Minor

tributaries .............................. 64-66

Kootenai River basin ................... 23-25

Legislation, appropriation

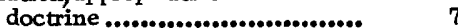

Carey Act of $1894 . \ldots \ldots \ldots \ldots \ldots \ldots \ldots \ldots . . . .17$

Reclamation Act of 1902............ 8

riparian doctrine........................ 6

Little Lost River ............................ 66

Lysimeter ................................. 13

Malheur River.............................. 81-84

Measurements of water consumption, methods, field plots............... 13-14 inflow and outflow, studies of....... 15, 18 lysimeter................................ 13 soil-moisture method................... 14 soil tanks .............................. 12-13

water table, fluctuations of ......... 14

46-47 Methow River.............................. 42-44

11 Minor tributaries, Neeley to Fing

10, 11 Hill...................................... 64-66

Missoula, Mont. , Clark Fork

Clark Fork below........................ Mud Lake area.............................. Murphy, King Hill to, Snake R'ver... Murphy to Weiser, Snake Rivem.......

Neeley to King Hill, minor tributaries ............................... 64-66

\section{Okanogan River}

40-42

$73-77$

Oxbow to Clarkston, Snake River..... 106-120

Oxbow, Weiser to, Snake Rive*....... 95-105

Payette River............................ 84-87

Pend Oreille River basin ................ $85-36$

Pend Oreille River below $Z$ Canyon near Metaline Falls, Wash ........ 32-35

Pine Creek ................................. 96-97

Portneuf River ........................... 63-64 
Powder River....................................

Reclamation Act of 1902

Return flow, mineral content of .....

Riparian doctrine ............................

St. Regis, Mont. , Clark Fork at ..... Salmon River ................................

Snake River ................................ above Heise ............................... at King Hill ............................... Grand Coulee to, Columbia River ...................................

Heise to King Hill .........................

King Hill to Murphy......................

Murphy to Weiser...........................

Oxbow to Clarkston.......................

Weiser to Oxbow .........................

Snake River and Yakima River,

Columbia River between ............

Soil-moisture method of water consumption measurement.............. Soil tanks, experiments with for water consumption measurement.. Sources of data on irrigation

\section{Page}

93-95

$58-120$

58-60

$69-71$

40-56

62-66

$72-90$

90-95

$106-120$

95-105

57

Page

Spokane River............................... 38-39

The Dalles, Columbia River n:ar .... 121-122

Transpiration .............................. 11

Tributaries, minor, Neeley to King

Hill ................................... 64-66

Tributary basins, list of ................. 19-20

Trinidad, Columbia River at.......... $\quad 49-50$

Umatilla River............................ 110-111

Walla Walla River ......................... 108-109

Water table, fluctuations of ........... 14

Weiser River ................................. 88-90

Weiser to Murphy, Snake River....... 90-95

Weiser to Oxbow, Snake River ......... 95-105

Wenatchee River ........................... 47-49

Willow Creek ............................... 112-113

Yakima River ............................ 52-56

Yakima River and Snake River,

Columbia River between ............ 57

Z Canyon, Pend Oreille River

below ..................................... 32-35

U. S. GOVERNMENT PRINTING OFF'CE: $0-1953$ 


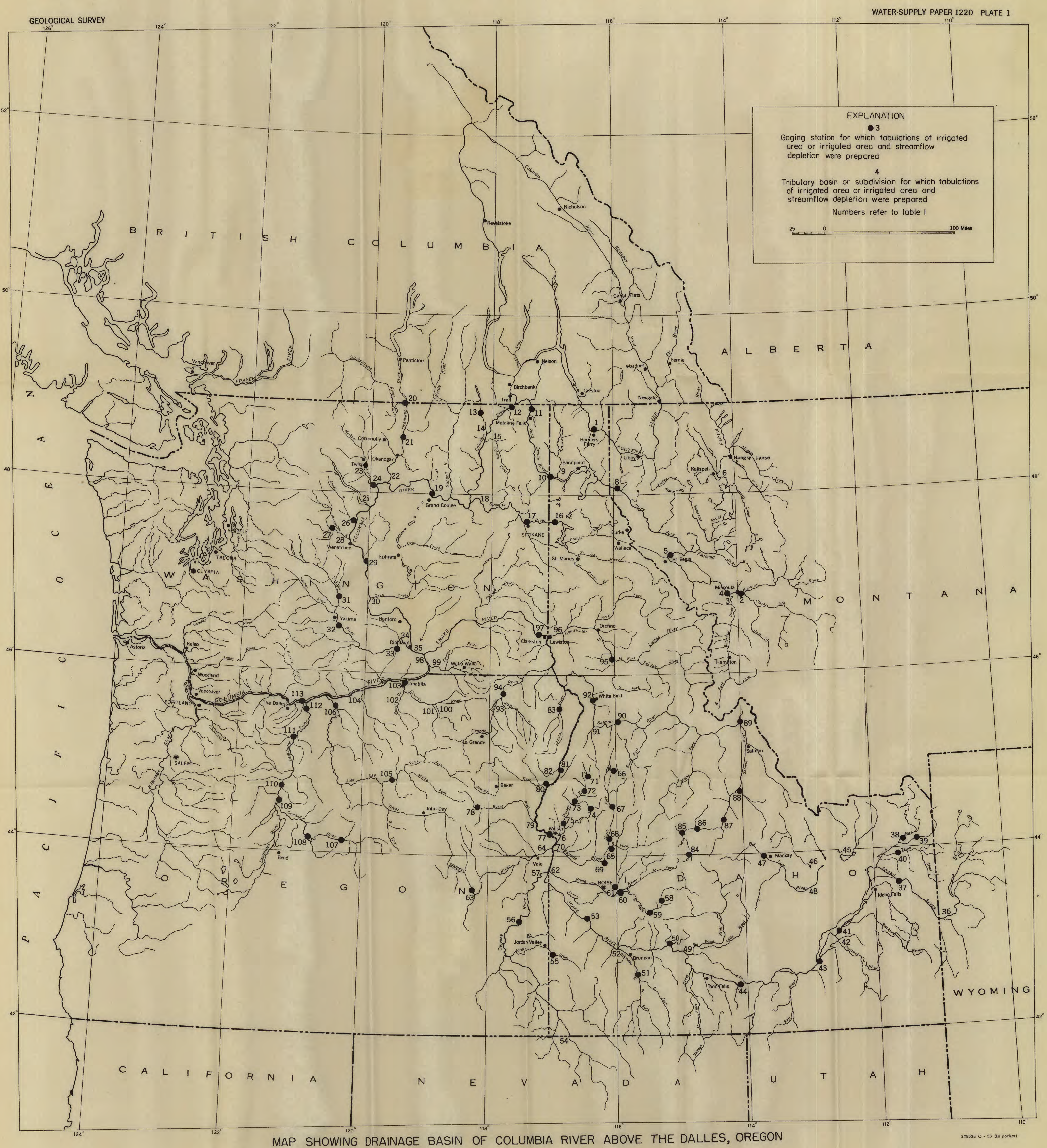

